\title{
A census of molecular hydrogen outflows and their sources along the Orion A molecular ridge
}

\section{Characteristics and overall distribution ${ }^{\star}$}

\author{
C. J. Davis ${ }^{1}$, D. Froebrich ${ }^{2}$, T. Stanke ${ }^{3}$, S. T. Megeath ${ }^{4}$, M. S. N. Kumar ${ }^{5}$, A. Adamson ${ }^{1}$, J. Eislöffel ${ }^{6}$, R. Gredel ${ }^{7}$, \\ T. Khanzadyan ${ }^{8}$, P. Lucas ${ }^{9}$, M. D. Smith ${ }^{2}$, and W. P. Varricatt ${ }^{1}$ \\ 1 Joint Astronomy Centre, 660 North A’ohōkū Place, University Park, Hilo, Hawaii 96720, USA \\ e-mail: c.davis@jach.hawaii.edu \\ 2 Centre for Astrophysics \& Planetary Science, School of Physical Sciences, University of Kent, Canterbury CT2 7NR, UK \\ 3 European Southern Observatory, Garching, Germany \\ 4 Department of Physics and Astronomy, University of Toledo, Toledo, OH 43606-3390, USA \\ 5 Centro de Astrofisica da Universidade do Porto, Rua das Estrelas s/n 4150-762 Porto, Portugal \\ 6 Thüringer Landessternwarte Tautenburg, Sternwarte 5, 07778 Tautenburg, Germany \\ 7 Max Plank Institute für Astronomie, Königstuhl 17, 69117 Heidelberg, Germany \\ 8 Centre for Astronomy, Department of Experimental Physics, National University of Ireland, Galway, Ireland \\ 9 Centre for Astrophysics Research, Science \& Technology Research Institute, University of Hertfordshire, College Lane, \\ Hatfield AL10 9AB, UK
}

Received 6 October 2008 / Accepted 16 December 2008

\begin{abstract}
Aims. A census of molecular hydrogen flows across the entire Orion A giant molecular cloud is sought. With this paper we aim to associate each flow with its progenitor and associated molecular core, so that the characteristics of the outflows and outflow sources can be established.

Methods. We present wide-field near-infrared images of Orion A, obtained with the Wide Field Camera, WFCAM, on the United Kingdom Infrared Telescope. Broad-band $\mathrm{K}$ and narrow-band $\mathrm{H}_{2} 1-0 \mathrm{~S}(1)$ images of a contiguous $\sim 8$ square degree region are compared to mid-IR photometry from the Spitzer Space Telescope and (sub)millimetre dust-continuum maps obtained with the MAMBO and SCUBA bolometer arrays. Using previously-published $\mathrm{H}_{2}$ images, we also measured proper motions for $\mathrm{H}_{2}$ features in 33 outflows, and use these data to help associate flows with existing sources and/or dust cores.

Results. Together these data give a detailed picture of dynamical star formation across this extensive region. We increase the number of known $\mathrm{H}_{2}$ outflows to 116 . A total of $111 \mathrm{H}_{2}$ flows were observed with Spitzer; outflow sources are identified for 72 of them (12 more $\mathrm{H}_{2}$ flows have tentative progenitors). The MAMBO $1200 \mu \mathrm{m}$ maps cover $97 \mathrm{H}_{2}$ flows; 57 of them (59\%) are associated with Spitzer sources and either dust cores or extended $1200 \mu \mathrm{m}$ emission. $\mathrm{The}_{2} \mathrm{H}_{2}$ jets are widely distributed and randomly orientated. The jets do not appear to be orthogonal to large-scale filaments or even to the small-scale cores associated with the outflow sources (at least when traced with the $11^{\prime \prime}$ resolution of the $1200 \mu \mathrm{m}$ MAMBO observations). Moreover, $\mathrm{H}_{2}$ jet lengths $(L)$ and opening angles $(\theta)$ are not obviously correlated with indicators of outflow source age - source spectral index, $\alpha$ (measured from mid-IR photometry), or (sub)millimetre core flux. It seems clear that excitation requirements limit the usefulness of $\mathrm{H}_{2}$ as a tracer of $L$ and $\theta$ (though jet position angles are well defined).

Conclusions. We demonstrate that $\mathrm{H}_{2}$ jet sources are predominantly protostellar sources with flat or positive mid-IR spectral indices, rather than disc-excess (or T Tauri) stars. Most protostars associated with molecular cores drive $\mathrm{H}_{2}$ outflows; however, not all molecular cores are associated with protostars or $\mathrm{H}_{2}$ jets. On statistical grounds, the $\mathrm{H}_{2}$ jet phase may be marginally shorter than the protostellar phase, though it must be considerably (by an order of magnitude) shorter than the prestellar phase. In terms of range and mean value of $\alpha, \mathrm{H}_{2}$ jet sources are indistinguishable from protostars. The spread in $\alpha$ observed for both protostars and $\mathrm{H}_{2}$ outflow sources is probably a function of inclination angle as much as source age. The few true protostars without $\mathrm{H}_{2}$ jets are almost certainly more evolved than their $\mathrm{H}_{2}$-jet-driving counterparts, although these later stages of protostellar evolution (as the source transitions to being a "disc-excess" source) must be very brief, since a large fraction of protostars do drive $\mathrm{H}_{2}$ flows. We also find that the protostars that power molecular outflows are no more (nor no less) clustered than protostars that do not. This suggests that the $\mathrm{H}_{2}$ emission regions in jets and outflows from young stars weaken and fade very quickly, before the source evolves from protostar to pre-mainsequence star, and on time-scales much shorter than those associated with the T Tauri phase, the Herbig-Haro jet phase, and the dispersal of young stellar objects.
\end{abstract}

Key words. stars: formation - stars: winds, outflows - ISM: Herbig-Haro objects - ISM: jets and outflows - infrared: ISM shock waves

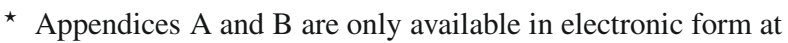
http://www . aanda.org
}

\section{Introduction}

The southern part of the Orion constellation encompasses the Orion A and B Giant Molecular Clouds (GMCs), numerous 
compact and intermediate-sized molecular cores, low and high mass young stars (including a massive star/young OB cluster), stars at varying evolutionary stages, and dozens of optical Herbig-Haro $(\mathrm{HH})$ objects and molecular outflows (see Peterson \& Megeath 2008; and Allen \& Davis 2008, for reviews). In molecular line maps the complex extends roughly northwest-southeast, parallel with the Galactic plane, over about $13^{\circ}$ (Kutner et al. 1977; Sakamoto et al. 1994). Overall, the Orion A and B GMCs represent one of the richest star forming regions known.

In extensive regions like Orion, jets and outflows can be used as sign-posts of on-going star formation. An abundance of jets points to active accretion and a young stellar population; a paucity of molecular outflows, in a region where near- and midIR photometry still indicate a sizable population of sources with reddening and excess, suggests a more evolved region with a fraction of pre-main-sequence stars ( $\mathrm{T}$ Tauri and Herbig Ae/Be stars) observed "edge-on" through their circumstellar discs.

By using $\mathrm{H}_{2} 2.122 \mu \mathrm{m}$ emission as a tracer of jets and outflows, wide-field narrow-band images may be used to pin-point the locations of the youngest sources. Moreover, they allow one to take a statistical approach when considering questions about the distribution of Class 0/I protostars and Class II/III Young Stellar Objects (YSOs), the interaction of these forming stars with their surrounding environment, the overall star formation efficiency, and the evolution of the region as a whole (e.g. Stanke 2000; Stanke et al. 2002; Davis et al. 2007; Kumar et al. 2007; Davis et al. 2008).

With the wide-field near-IR camera WFCAM at the United Kingdom Infrared Telescope (UKIRT) we have secured homogeneously deep, sub-arcsecond-resolution $\mathrm{H}_{2} 2.122 \mu \mathrm{m}$ and broad-band $\mathrm{K}$ images over most of the Orion $\mathrm{A} G M C^{1}$. Our $\sim 8$ square degree mosaics encompass the the molecular clouds known as OMC 2 and OMC 3, the Orion Nebula Cluster (ONC also known as M 42), and an abundance of well-known low mass star forming cores, $\mathrm{HH}$ objects and bipolar outflows spread throughout the Lynds dark cloud L 1641 (HH 1/2, HH 33/40, $\mathrm{HH} 34$, HH 38/43, etc.).

Our goals with these observations were to: (1) extend the maps of Stanke et al. (2002; hereafter Sta02) to give more complete coverage of Orion $\mathrm{A}$, to search for new $\mathrm{H}_{2}$ flows, and to better trace the true extent of known $\mathrm{H}_{2}$ flows by searching for emission off the main molecular ridge; (2) provide a second epoch for proper motion studies, and (3) compare the observations with extensive (sub)millimetre and mid-IR Spitzer observations, so that $\mathrm{H}_{2}$ jets and outflows could be associated with molecular cores and/or embedded protostars, the latter being identified from mid-IR photometry. We utilise the $850 \mu \mathrm{m}$ SCUBA data published by Nutter \& Ward-Thompson (2007), the more extensive $1200 \mu \mathrm{m}$ MAMBO observations of Stanke et al. (in prep.), and the recent Spitzer observations of Megeath et al. (in prep.).

This paper is structured as follows: in Sect. 3 we briefly discuss wide-field images of regions of interest spread throughout Orion A and give statistical information pertaining to the overall population of outflows. In Tables 2 and 3 we list the $\mathrm{H}_{2}$ flows catalogued by $\mathrm{Sta} 02$ and the newly identified $\mathrm{H}_{2}$ flows from this paper, respectively: in both tables we list the likely outflow source (if known), the dense cores that coincide with the source, and any $\mathrm{HH}$ objects that are associated with the flow. In Sect. 4. we discuss the region as a whole, drawing statistical information

\footnotetext{
${ }^{1} \mathrm{H}_{2}$ and K-band images in Rice-compressed FITS format are available from http://www . jach.hawaii . edu/UKIRT/TAP/
}

from the sample of flows and associated sources. In Appendix A we briefly discuss the full sample of molecular $\mathrm{H}_{2}$ outflows, presenting continuum-subtracted $\mathrm{H}_{2} 2.122 \mu \mathrm{m}$ images of the newlyobserved flows. In Appendix B we discuss our proper motion measurements for outflows observed here and in the earlier work of $\mathrm{Sta} 02$.

\section{Observations}

\subsection{Near-IR imaging}

Broad-band $J, H, K$ and narrow-band $\mathrm{H}_{2} 2.122 \mu \mathrm{m}$ images of a $1.5^{\circ} \times 1.5^{\circ}$ field centred on M 42/M 43 were obtained during instrument commissioning on 24 November 2004 (filter characteristics are described in Hewett et al. 2006). Broad-band $K$ and narrow-band $\mathrm{H}_{2}$ images of a $\sim 8$ square degree field were later secured during Director's Discretionary Time (DDT), on 15-16 December 2005. $\mathrm{K}$ and $\mathrm{H}_{2}$ images of a single 0.75 square degree segment centred on the Orion nebula were also obtained during Service observing time on 12 February 2007. The commissioning data have only been used to construct the colour images in Fig. 2; the later observations, which were secured under slightly better observing conditions, and when the instrument was better characterised, have been used for all other figures and analysis. The service data have been used to fill in a gap in the DDT data, where bright stars and saturation affects produced poor results in the processing.

The near-IR wide-field camera WFCAM (Casali et al. 2007) at UKIRT was used on each occasion. WFCAM houses four Rockwell Hawaii-II $(\mathrm{HgCdTe} 2048 \times 2048)$ arrays spaced by $94 \%$ in the focal plane. The pixel scale measures $0.40^{\prime \prime}$. To observe a contiguous square field on the sky covering 0.75 square degrees - a WFCAM "tile" - observations at four positions are required. At each position, to correct for bad pixels and array artifacts, a five-point jitter pattern was executed (with offsets of $3.2^{\prime \prime}$ or $6.4^{\prime \prime}$ ); to fully sample the seeing, at each jitter position a $2 \times 2$ micro-stepped pattern was also used, with the array shifted by odd-integer multiples of half a pixel. 20 frames were thus obtained at each of the four positions in the tile. Eleven tiles in total were observed covering over 8 square degrees, through both broad-band $K$ and narrow-band $\mathrm{H}_{2}$ 1-0S(1) filters. Exposure times of $5 \mathrm{~s} \times 2$-coadds and $20 \mathrm{~s} \times 2$-coadds were used with the $K$ and $\mathrm{H}_{2}$ filters, respectively. With the jitter pattern and microstepping the total on-source/per-pixel integration time in $K$ was therefore $200 \mathrm{~s}$; in $\mathrm{H}_{2}$ the total exposure time was $800 \mathrm{~s}$.

The DDT data were reduced by the Cambridge Astronomical Survey Unit (CASU), which is responsible for data processing prior to archiving and distribution by the Wide Field Astronomy Unit (WFAU). However, the processing of frames that included the bright Orion Nebula and Trapezium cluster gave images with severe background structure, probably related to the adopted method for sky-subtraction. Our service observations of this central region were therefore reduced using the ORAC-DR pipeline at the telescope (Cavanagh et al. 2003), which performs flatfielding using twilight flats, but does not subtract a sky frame. The CASU reduction steps are described in detail by Dye et al. (2006). Residual sky/background structure was later removed by fitting a coarse surface to each image (as described by Davis et al. 2007). For both the CASU and Service data, astrometric and photometric calibrations were achieved using 2MASS (Dye et al. 2006; Hewett et al. 2006); the calibrated images were subsequently used to construct the large-scale mosaics presented in this paper. 

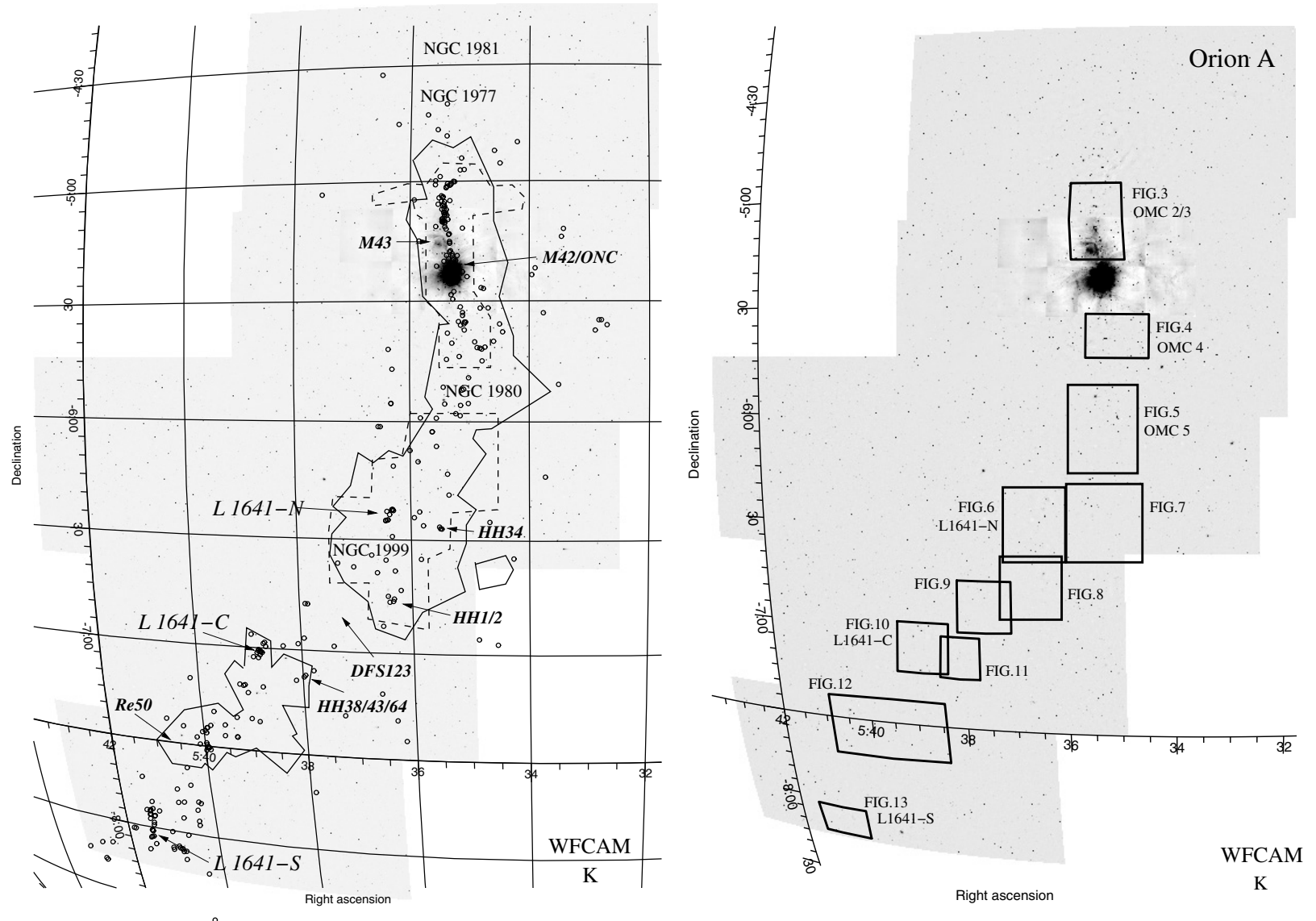

Fig. 1. Our near-IR $K$-band mosaic of Orion A. In the left-hand panel, the positions of candidate protostars identified from Spitzer photometry are marked with circles; the areas outlined with full lines have been mapped at $1200 \mu \mathrm{m}$ (Stanke et al., in prep.); the areas marked with dashed lines have been mapped at $850 \mu \mathrm{m}$ (Nutter \& Ward-Thompson 2007). In the right-hand panel the regions shown in detail in Figs. 3 to 13 are indicated with boxes.

In our $K$-band images we reach a limiting magnitude of $\sim 18.3$ in the less-nebulous regions; our sensitivity to point sources in the very bright Orion nebula regions is $2-3 \mathrm{mag}$ worse. In the $\mathrm{H}_{2}$ images of $\mathrm{L} 1641$, outflow features with a surface brightness of $\sim 7 \times 10^{-19} \mathrm{~W} \mathrm{~m}^{2} \operatorname{arcsec}^{-2}$ are detected at $3-5 \sigma$ above the surrounding background. The $\mathrm{H}_{2}$ sensitivity in the ONC region is again lower because of the variable, diffuse nebulosity.

An overview of the region observed with WFCAM is given in Fig. 1; colour images of the spectacular OMC 1/2/3 regions are presented in Fig. 2; large-scale $\mathrm{H}_{2} 1-0 \mathrm{~S}(1)$ mosaics of regions of interest throughout Orion A are presented in Figs. 3-13. Continuum-subtracted images of the newly-identified $\mathrm{H}_{2}$ flows are available in Appendix A. In all of these figures axes are labelled in 2000.0 coordinates.

\subsection{Proper motion measurements}

Using the newly-acquired WFCAM images and the $\mathrm{H}_{2}$ mosaics of Sta02, which were obtained between December 1996 and May 1998 with the 3.6-m telescope at the Calar Alto Observatory, Spain, we have measured tangential velocities for emission-line features in a number of flows. We focus on regions where multiple flows are observed, particularly where the association between outflows and embedded protostars is ambiguous.
A complete list of proper motions (PMs) is given in Appendix B, where the technique is described in detail.

\subsection{Mid-IR observations}

The Spitzer Space Telescope observations discussed in this paper were obtained with the IRAC and MIPS cameras (Fazio et al. 2004; Rieke et al. 2004). A full description of the data analysis and young stellar object identification will be presented in Megeath et al. (in prep.); we provide here a brief summary.

Images in IRAC bands 1, 2, 3 and 4 (at 3.6, 4.5, 5.8 and $8.0 \mu \mathrm{m}$ ) and MIPS band 1 (at $24 \mu \mathrm{m}$ ) were used to compile a list of more than 300 candidate protostars between declinations $-4.6^{\circ}$ and $-9.0^{\circ}$. The protostars were selected from their [3.6]-[4.5] and [4.5]-[24] colours (Megeath et al. 2009), although note that the majority of these sources would also be classified as flat spectrum, Class 0 or Class I protostars if one used their Spectral Energy Distributions (SEDs). To minimise contamination from extragalactic sources we rejected all sources with protostellar colours but $24 \mu \mathrm{m}$ magnitudes fainter than 7 . About $20 \%$ of the protostars lacked usable $24 \mu \mathrm{m}$ data and were therefore identified through their [4.5]-[5.8] colour using the approach of Gutermuth et al. (2008); most of these sources are found in the Orion nebula where the $24 \mu \mathrm{m}$ data were saturated.

In the list of protostars there remains a small amount of contamination from extragalactic sources, particularly AGN; we 

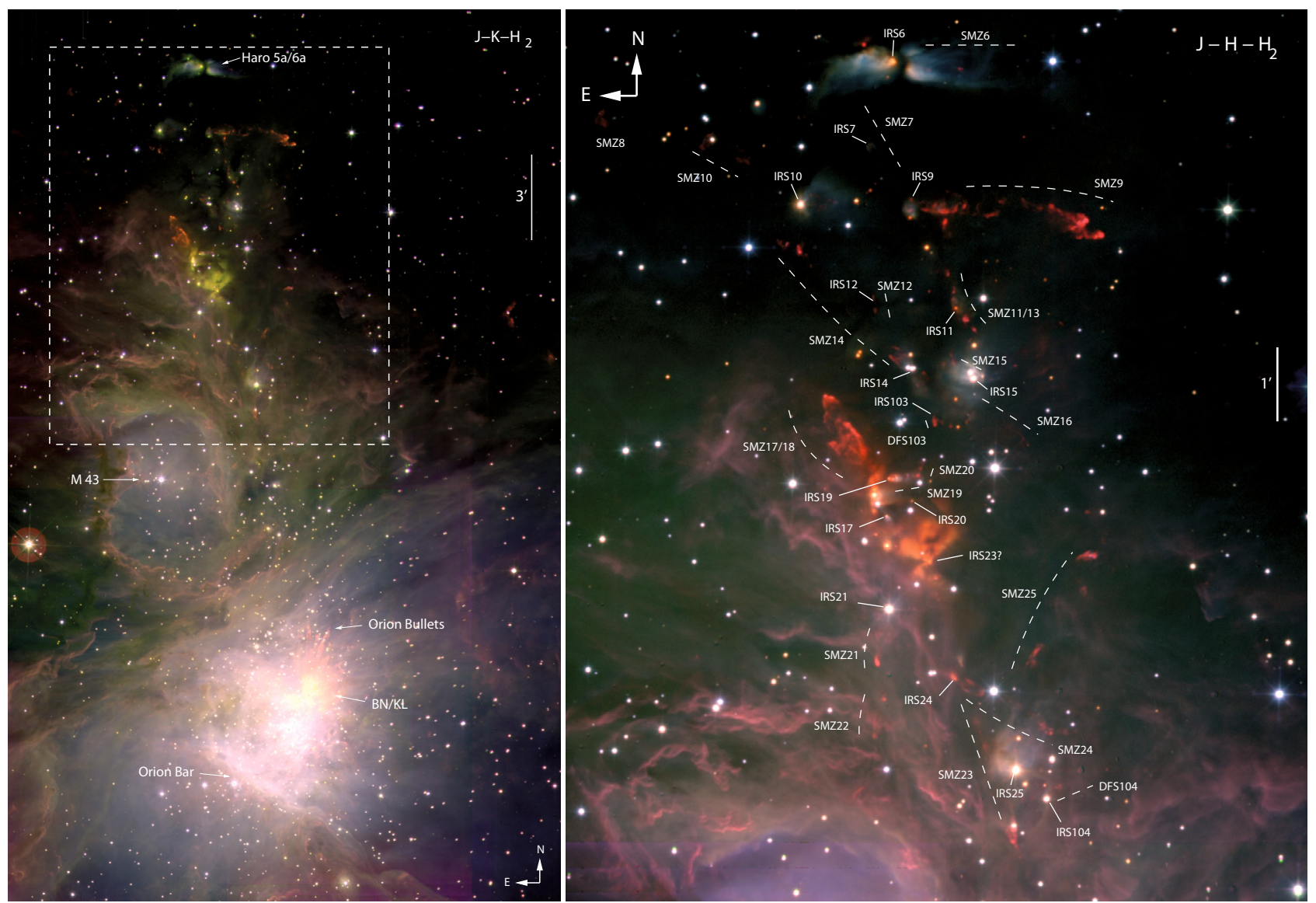

Fig. 2. Left: colour image of OMC 1/2/3 composed of broad-band $J, K$ and narrow-band $\mathrm{H}_{2} 2.122 \mu \mathrm{m}$ observations. The data have been stretched logarithmically. Right: colour $J, H$ and narrow-band $\mathrm{H}_{2} 2.122 \mu \mathrm{m}$ composite of the region between Haro 5a/6a and M 43 (OMC 2/3). The data have again been stretched logarithmically. $\mathrm{H}_{2}$ flows are labelled with "SMZ" or "DFS" numbers; outflow sources are labelled with "IRS" numbers. The dashed box in the left-hand image marks the area covered by the right-hand image.

estimate this contamination to be 0.7 sources per sq. degree. In addition, there may also be some contamination from embedded Class II sources and potentially from edge-on young stars with flared discs. Estimates of the degree of contamination await the analysis of recent Spitzer 5-40 $\mu \mathrm{m}$ spectroscopy of the sample.

In addition to the protostars, a further $\sim 2000$ disc-excess sources were identified in the Spitzer data.

The sample of protostars is not complete: we expect that many protostars are hidden in the bright nebulosity toward the Orion nebula, and that several very bright prototstars were omitted because of strong saturation.

To characterise the SED of each protostar and disc-excess source, a spectral index was obtained by fitting a single powerlaw to the $3.6,4.5,5.8,8$ and $24 \mu \mathrm{m}$ photometry. For sources without $24 \mu \mathrm{m}$ detections, the spectral index was calculated from just the IRAC photometry. The resulting 3.6-24 $\mu \mathrm{m}$ spectralindex, $\alpha=\mathrm{d} \log (\lambda F(\lambda)) / \mathrm{d} \log \lambda$, ranges from -0.5 to 3.0 for the protostars, and from -2.5 to 0.0 for the disc-excess sources. Although the mid-IR spectral index is a useful indicator of evolution, the index does depend on the density, the rotation rate, and the inclination of the protostellar envelope (Whitney et al. 2003, 2004). Consequently, one should not expect a fool-proof relationship between $\alpha$ and the age of the protostar. A histogram showing the distribution of $\alpha$ for the protostars in Orion peaks at around 0 and gradually declines with increasing $\alpha$; a histogram of $\alpha$ values for the disc-excess sources peaks at around -0.8 , consistent with a large population of Class II objects (Fig. 17). Note that the spectral indices of the disc excess sources can be affected by extinction. Hence, the distribution of their $\alpha$ values overlaps the distribution of $\alpha$ for the protostars (there is not a discontinuity in the distribution of $\alpha$ between the disc-excess and protostars). This may reflect a continuous transition between protostars and disc-excess sources, as the infalling envelope is dissipated and the inner star/disc system is revealed.

\section{4. (Sub)millimetre observations}

In many of the subsequent figures we also overlay contours of dust continuum emission at $1200 \mu \mathrm{m}$. These data were obtained with the IRAM 30-m telescope at Pico Veleta, Spain, with the 37 and 117-pixel Max Planck Millimeter Bolometer (MAMBO) arrays (Kreysa et al. 1999). The data were acquired over several observing runs between 1999 and 2002, and reduced using the in-house reduction package "MOPSI". Standard reduction steps were employed; baseline-fitting, de-spiking, correction for atmospheric extinction and flux calibration using planetary observations. At IRAM the MAMBO Half Power Beam Width (HPBW) measures $11^{\prime \prime}$.

A preliminary catalogue of $\sim 500$ MAMBO cores was obtained from visual inspection of the map and 2-dimensional Gaussian fitting of features. From this list we identify cores that coincide with an $\mathrm{H}_{2}$ outflow source and list the core parameters 


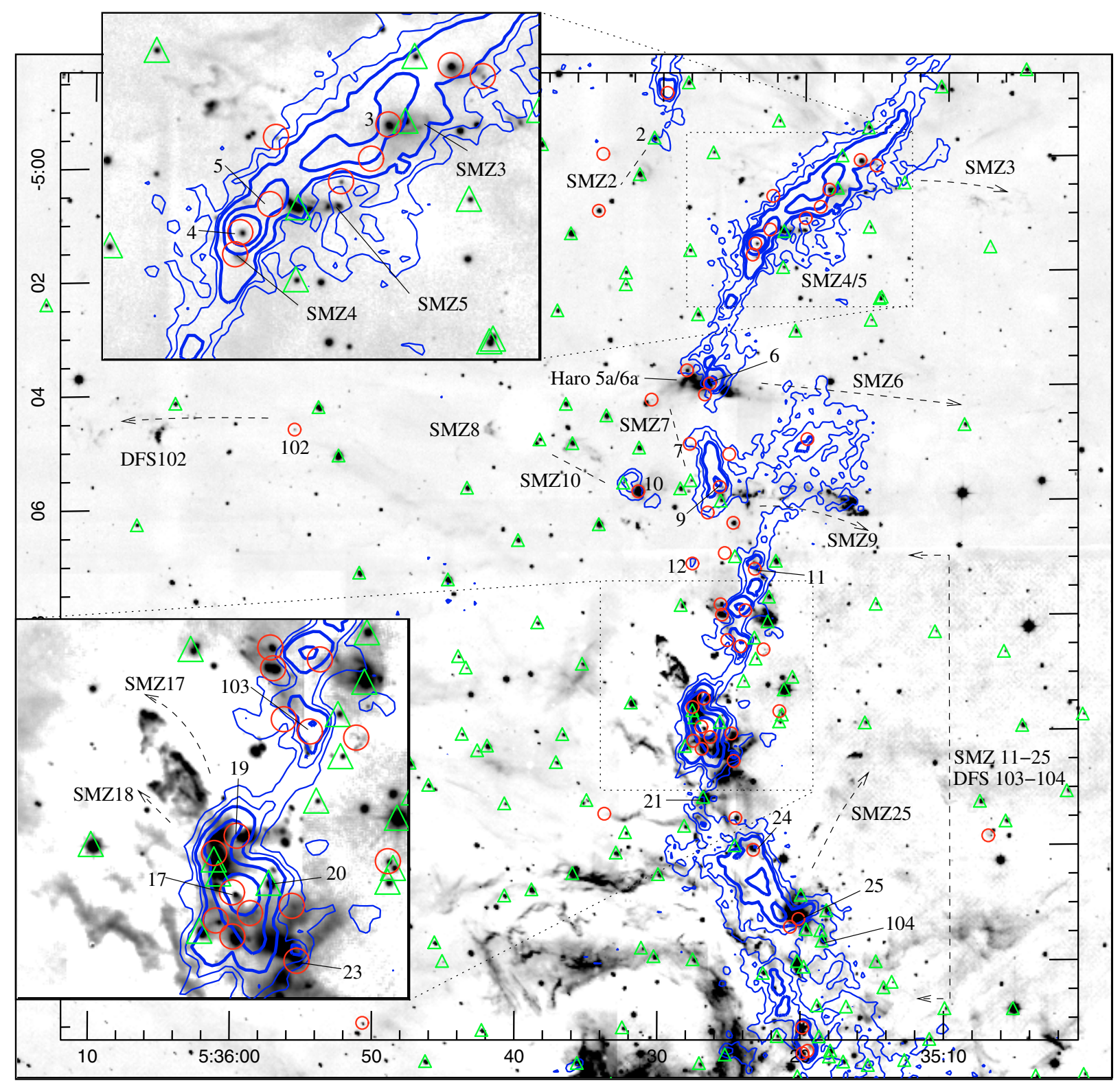

Fig. 3. Narrow-band $\mathrm{H}_{2} 2.122 \mu \mathrm{m}$ images of OMC 2/3, the region directly north of the ONC in Fig. 1 (inset figures are double the scale and displayed with a logarithmic stretch). Here and elsewhere in this paper Spitzer protostars and disc sources are marked with circles and triangles,

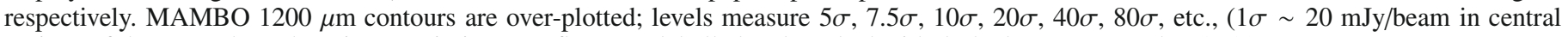
regions of the map where there is no emission). $\mathrm{H}_{2}$ flows are labelled and marked with dashed arrows (see also Sta02).

in Tables 2 and 3. The MAMBO data-set will be analysed in detail in a future paper (Stanke et al., in prep.).

\section{Results}

\subsection{Overview}

In Fig. 1 we present a mosaic of the complete $K$-band data-set. We indicate on this figure the extent of the $850 \mu \mathrm{m}$ and $1200 \mu \mathrm{m}$ maps, and mark the positions of $\sim 300$ protostars.

Colour images of the bright, nebulous regions around the ONC/M 42, the M 43 HII region, and the complex star forming environment around Haro $5 \mathrm{a} / 6 \mathrm{a}$ are shown in Fig. 2. These allow the reader to better distinguish $\mathrm{H}_{2}$-emitting jets and outflows from filaments of bright nebulosity. Near-IR observations of the region around $\mathrm{BN} / \mathrm{KL}$, including the spectacular Orion Bullets, the Orion Bar, BN and the Trapezium cluster, have been discussed in many other papers (e.g. Usuda et al. 1996; Schild et al. 1997; Lee \& Burton 2000; Bally et al. 2000; Carpenter et al. 2001; Slesnick et al. 2004; Kassis et al. 2006; Simpson et al. 2006; Tamura et al. 2006). In this paper we therefore focus on the area to the north of the ONC, the OMC $2 / 3$ region, and the extensive L 1641 low-mass star forming region to the south.

In Figs. 3-13 we present segments of the full $\mathrm{H}_{2}$ mosaic, indicating with dashed lines the locations of $\mathrm{H}_{2}$ jets and outflows (the data in these figures have been binned over $4 \times 4$ pixels, to a pixel scale of $0.8^{\prime \prime}$, to enhance the fainter features). Many of the outflows imaged here have already been catalogued by $\mathrm{Sta} 02\left(\mathrm{H}_{2}\right.$ images of OMC $2 / 3$ are also presented by $\mathrm{Yu}$ et al. 1997, 2000). With the newly-identified $\mathrm{H}_{2}$ flows, we therefore continue the numbering scheme of Sta02, although new flows (often found beyond the bounds of the Sta02 data) are numbered above 100. The Sta02 objects and the new $\mathrm{H}_{2}$ outflows are prefixed with SMZ and DFS, respectively. Note that entire 

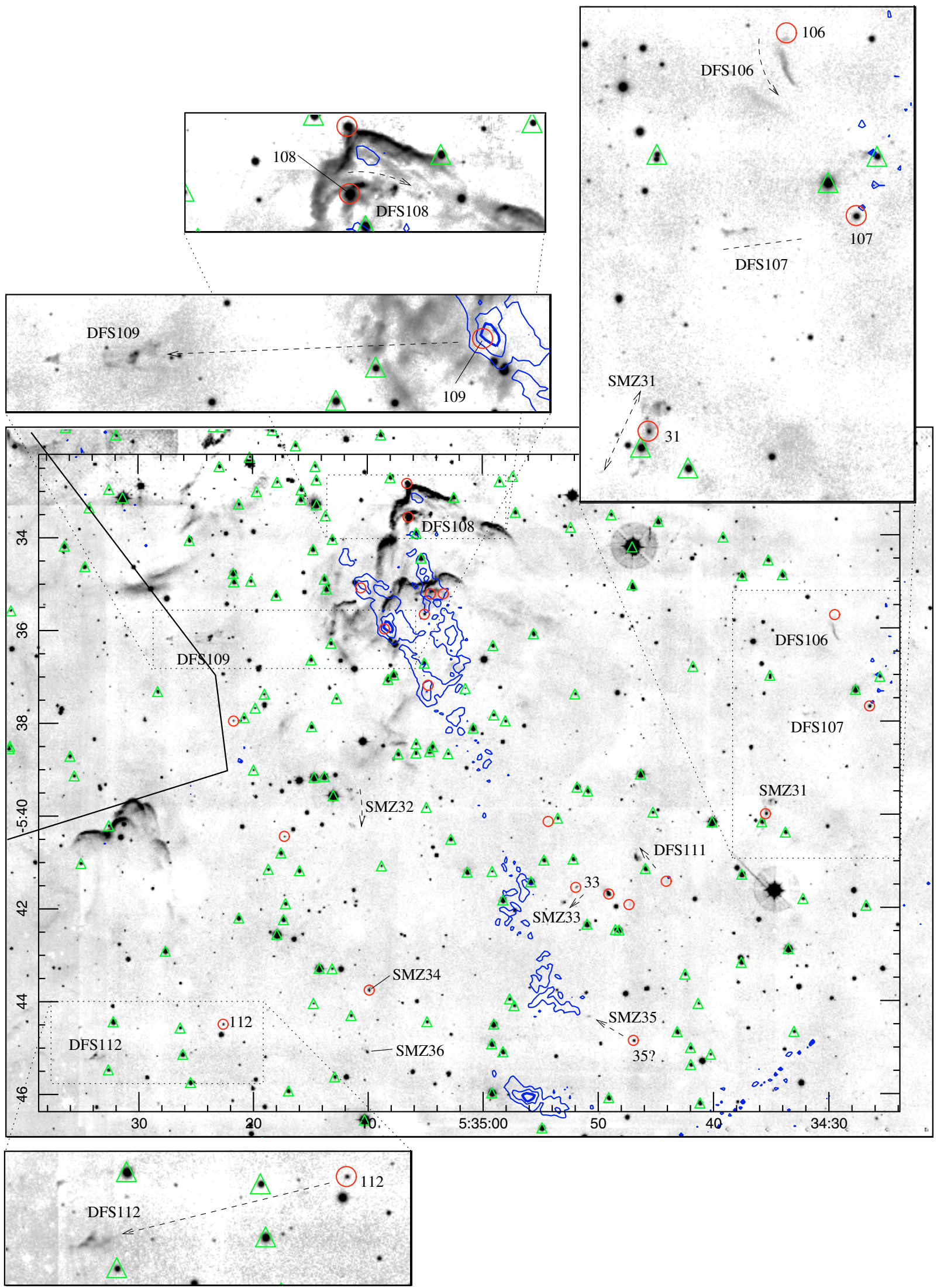

Fig. 4. Narrow-band $\mathrm{H}_{2} 2.122 \mu \mathrm{m}$ images of the region $20^{\prime}$ south of the ONC (Johnstone \& Bally 2006, refer to this region as OMC 4). Inset figures are again double the scale and displayed with a logarithmic stretch. The full line on the left marks the edge of the MAMBO map. DFS 105, DFS 110 and DFS 114 lie to the west of the region shown above. Contours and symbols are as in Fig. 3. 


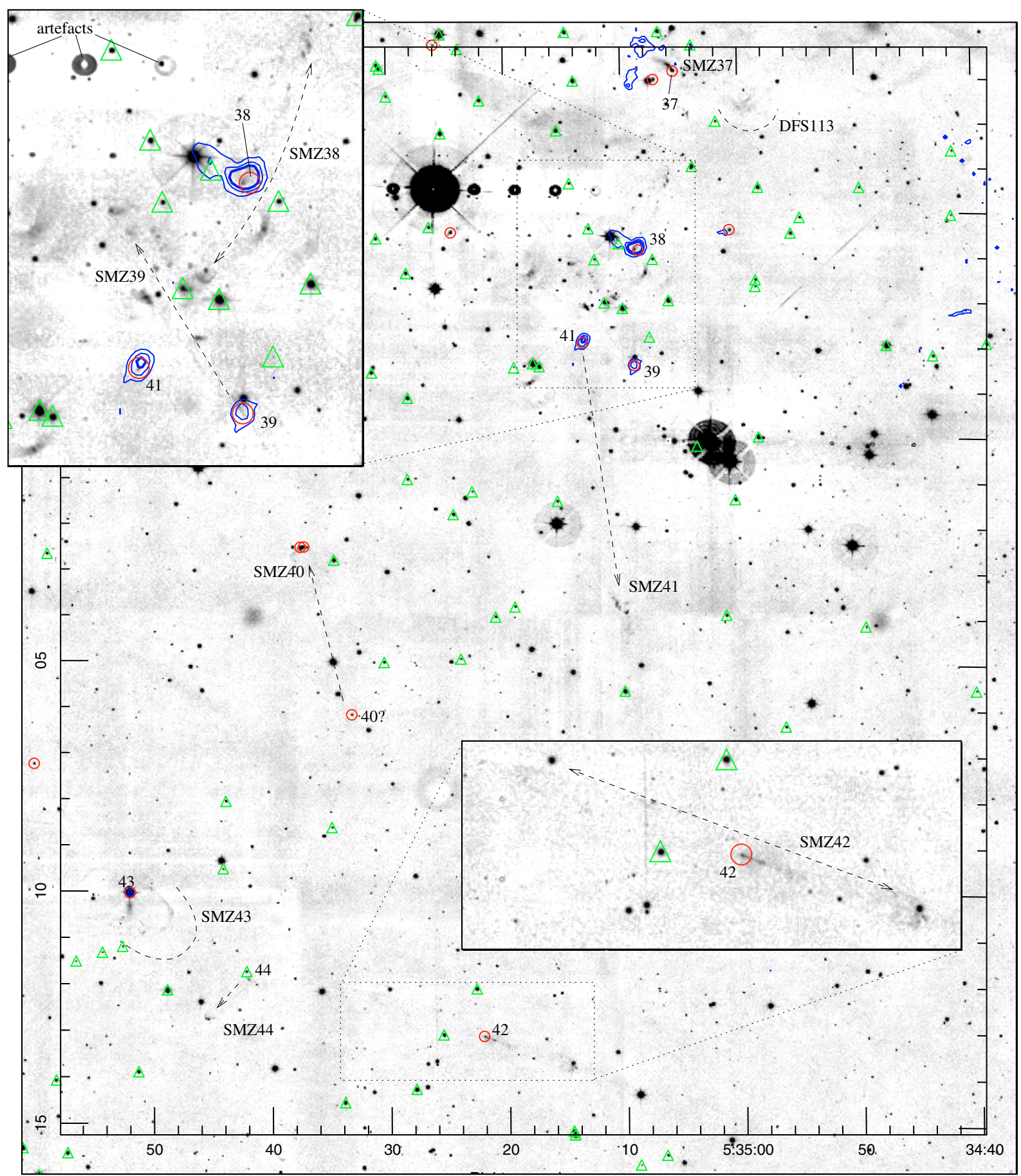

Fig. 5. Narrow-band $\mathrm{H}_{2} 2.122 \mu \mathrm{m}$ images of the region around NGC 1980 - OMC 5 in Johnstone \& Bally (2006) - about 45' south of the ONC (see Fig. 1). Inset figures are again double the scale and displayed with a logarithmic stretch. Contours and symbols are as in Fig. 3.

$\mathrm{H}_{2}$ flows, rather than individual $\mathrm{H}_{2}$ features or small groups of knots, are given a single number. For example, $\mathrm{HH} 1$ and $\mathrm{HH} 2$, which are relatively discrete objects, are here (and in Sta02) referred to as SMZ 64. All $\mathrm{H}_{2}$ flows are listed in Tables 2 and 3; continuum-subtracted $\mathrm{H}_{2}$ images of the new DFS flows are given in Appendix A, along with a brief description of each outflow.

In Figs. 3-13 we also mark the positions of the protostars and (more numerous) disc-excess sources identified from the Spitzer data; candidate outflow sources, when identified, are numbered using the same value as is given to the $\mathrm{H}_{2}$ outflow itself (so for example DFS 101 is driven by IRS 101). Outflow sources are listed in Tables 2 and 3, together with any coincident molecular cores seen at $850 \mu \mathrm{m}$ or $1200 \mu \mathrm{m}$. $850 \mu \mathrm{m}$ cores are taken from Nutter \& Ward-Thompson (2007); the table of $1200 \mu \mathrm{m}$ cores has yet to be published (Stanke et al., in prep.), so here we again use the outflow number, together with MMS (for MilliMetre Source), to identify each core.

The association of jets with young stars and/or (sub)millimetre continuum peaks is largely based on morphology and/or alignment. However, in some of the more complex regions we use the PM measurements in Appendix B to help isolate the location of the outflow source; in most cases 


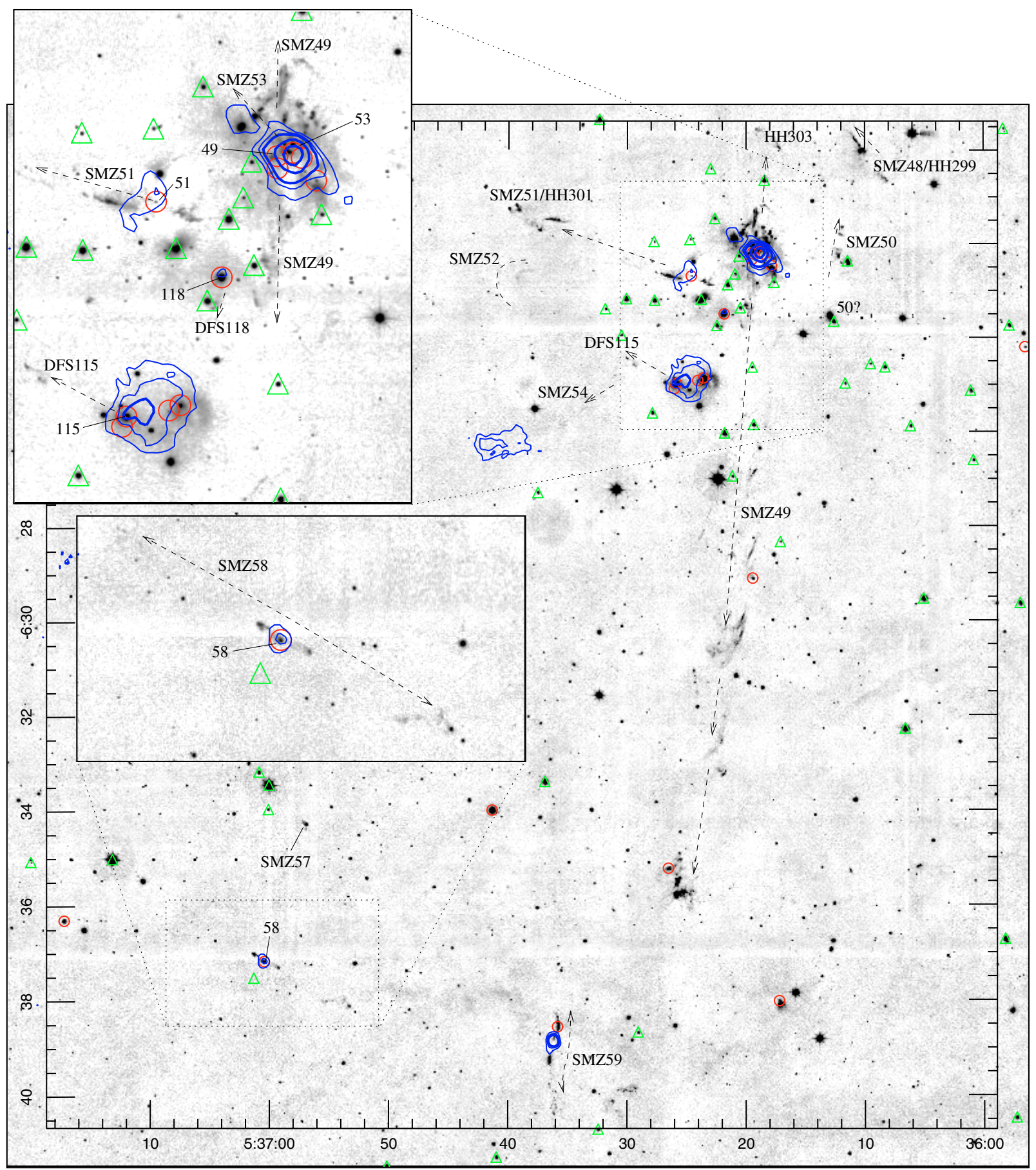

Fig. 6. Narrow-band $\mathrm{H}_{2} 2.122 \mu$ m images of L 1641-N (labelled in Fig. 1). Both insets are displayed at twice the scale and with a logarithmic stretch. Contours and symbols are as in Fig. 3.

the direction of propagation of the shock features are as one might expect, based on their morphology and the location of candidate outflow sources.

\subsection{Outflow activity in Orion $A$}

OMC 2/3 (Figs. 2 and 3), the region to the north of the ONC, is described in considerable detail by $\mathrm{Yu}$ et al. (2000). HH objects are identified by Reipurth et al. (1997) and Bally \& Devine (2001). High-resolution CO outflow maps are presented by Williams et al. (2003). The distribution of dense molecular gas throughout OMC $1 / 2 / 3$ has been mapped at $450 \mu \mathrm{m}$ and
$850 \mu \mathrm{m}$ by Johnstone \& Bally (1999); Chini et al. (1997) present $1300 \mu \mathrm{m}$ observations of OMC $2 / 3$. A comprehensive review is also given by Peterson \& Megeath (2008).

The vast majority of protostars in Fig. 3 are located within $11^{\prime \prime}$ of a MAMBO core and/or 14" of a SCUBA core (these radii being equivalent to the HPBW of these (sub)millimetre data). The association of bright $\mathrm{H}_{2}$ jets with Spitzer protostars and cold molecular cores attests to the youth of the outflows in this region. Most of the protostars are located along the chain of cores that runs through the centre of OMC $2 / 3$ (the disc excess sources are more widely distributed); similarly, most of the $\mathrm{H}_{2}$ outflows seem to be driven by sources close to this central axis. The most 


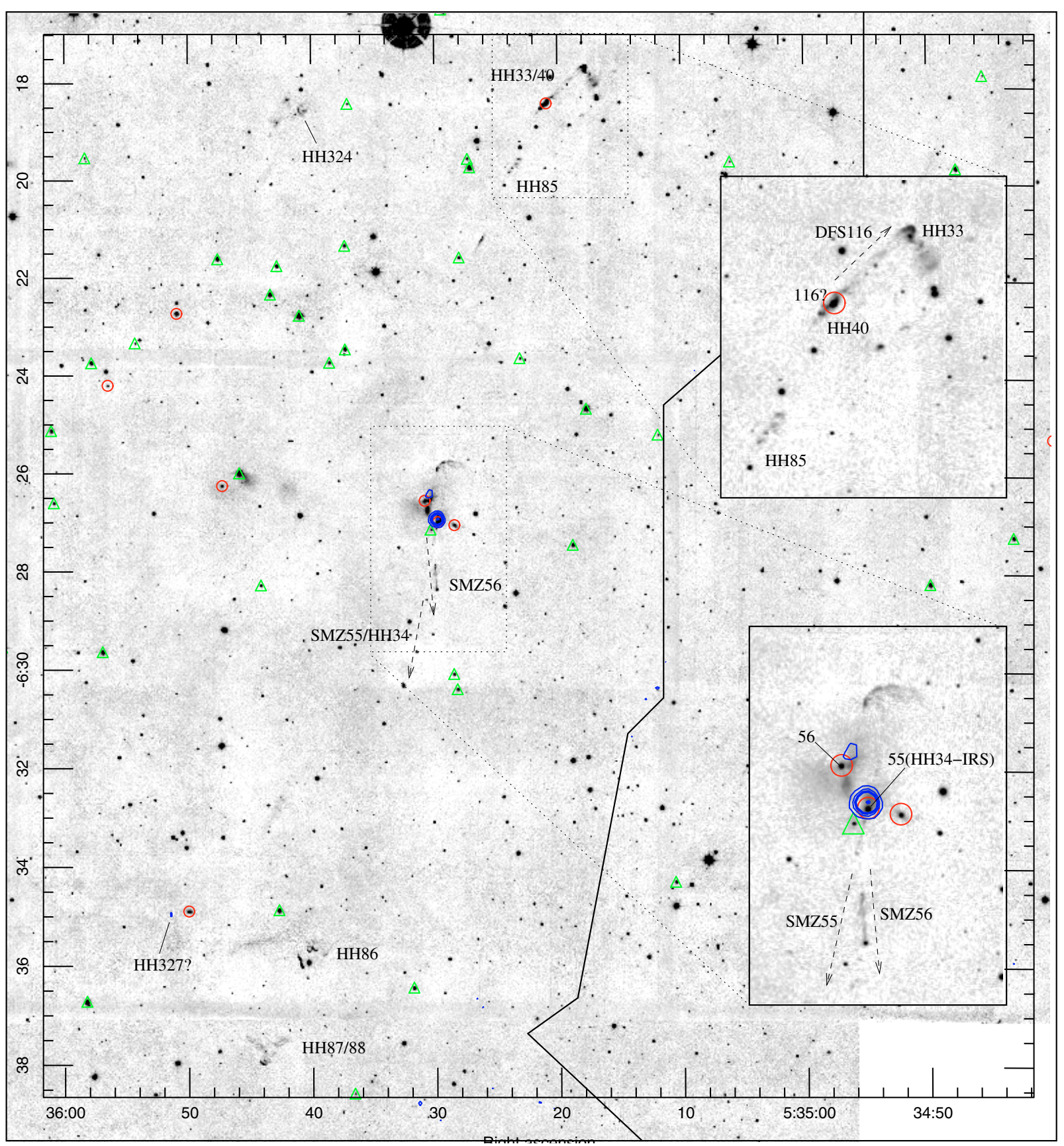

Fig. 7. Narrow-band $\mathrm{H}_{2} 2.122 \mu \mathrm{m}$ images of the region around $\mathrm{HH} 33 / 40$ and $\mathrm{HH} 34$ (roughly $0.5^{\circ}$ west of L 1641-N - see Fig. 1). The zig-zag line through the centre of the image marks the western border of the MAMBO map. Both insets are again displayed at twice the scale and with a logarithmic stretch. Contours and symbols are as in Fig. 3.

distinctive $\mathrm{H}_{2}$ flows, SMZ 3, SMZ 5, SMZ 6 and SMZ 9 are also orientated perpendicular to the north-south axis of cores.

From $\mathrm{Sta} 02$ there are $22 \mathrm{H}_{2}$ flows spread across the region shown in Fig. 3 (we regard SMZ 11 and SMZ 13 as a single flow; likewise SMZ 14/16 and SMZ 21/22). We identify four new $\mathrm{H}_{2}$ flows north of the ONC, DFS 101-104. Candidate outflow sources are found for 24 of the $26 \mathrm{H}_{2}$ flows. Only two have negative spectral indices; the rest are either "flat spectrum" or reddened protostars. Two of the 25 Spitzer outflow sources were not observed with MAMBO; of the remaining 23, 18 are associated with $1200 \mu \mathrm{m}$ (MMS) cores.

In Figs. 4 and 5 we show large-scale $\mathrm{H}_{2}$ images of two regions south of the ONC, adjacent to NGC 1980 in the overview plot in Fig. 1. Johnstone \& Bally (2006) refer to these regions as OMC 4 and OMC 5, respectively. In and around these two areas $\mathrm{Sta02}$ identify at least 18 possible $\mathrm{H}_{2}$ flows (SMZ 29-SMZ 46; between declination $-5^{\circ} 20^{\prime}$ and $-6^{\circ} 15^{\prime}$ ); we add a further ten $\mathrm{H}_{2}$ flows to this tally (DFS 105-114; note that DFS 105, DFS 110 and DFS 114 are beyond the edges of Fig. 4). Moreover, PMs have been measured for many of the flows in this area.

We identify candidate outflow sources for 25 of the 28 outflows in the OMC 4 and 5 regions, although unlike OMC 2/3, many of these sources are not associated with dust cores. (Note that Johnstone \& Bally 2006 also compare $\mathrm{H}_{2}$ images with dust continuum maps in this region, using the images of 


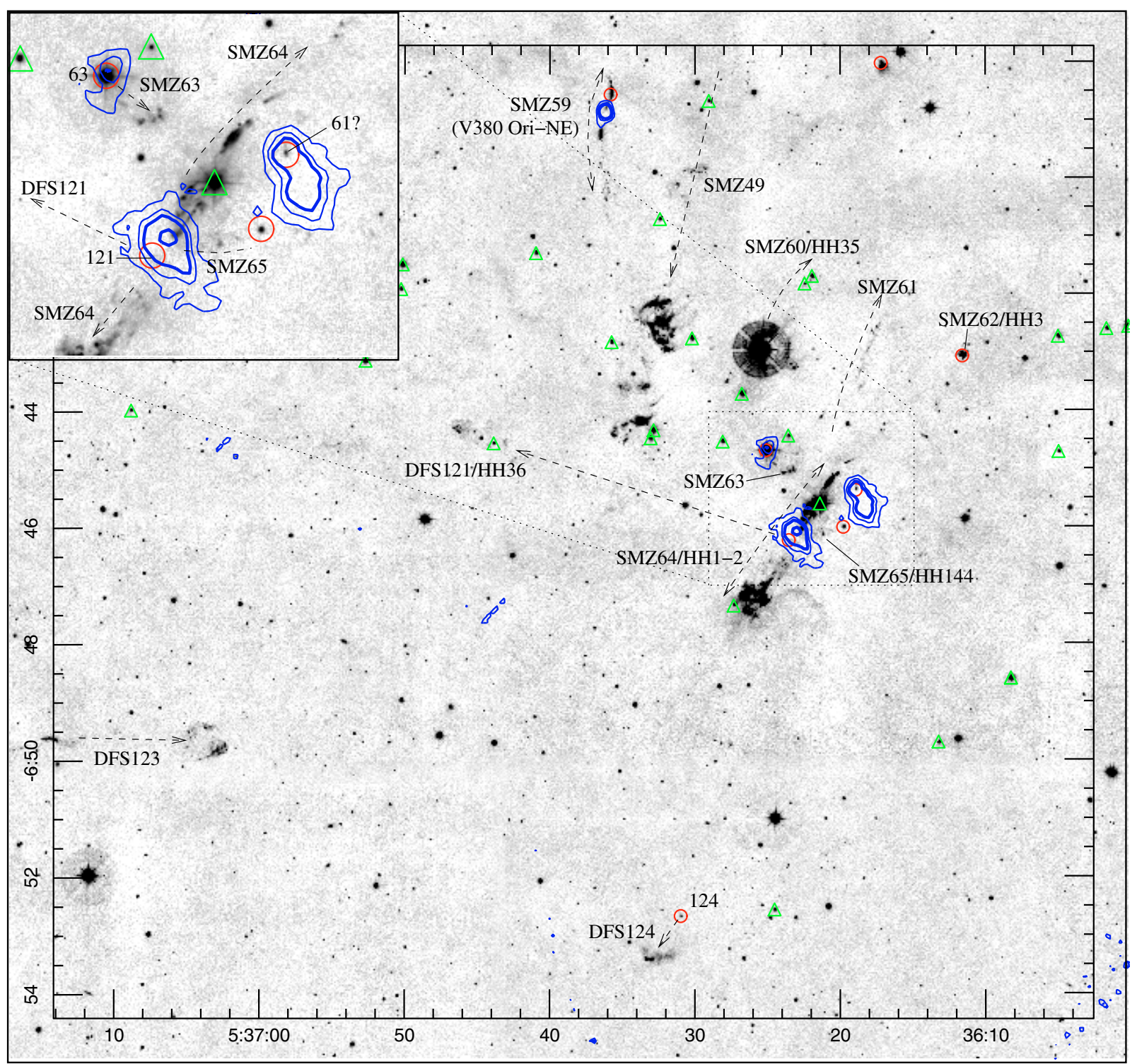

Fig. 8. Narrow-band $\mathrm{H}_{2} 2.122 \mu \mathrm{m}$ images of the region around $\mathrm{HH} 1 / 2$ in NGC 1999 (roughly $0.5^{\circ}$ south of L 1641-N - see Fig. 1). The inset of HH 1 is double-scale and displayed with a logarithmic stretch. Contours and symbols are as in Fig. 3.

Sta02 and their SCUBA observations, respectively.) All of the SMZ jets were observed with MAMBO, as were seven of the 10 DFS flows. However, only 14 of the 25 observed $\mathrm{H}_{2}$ outflow sources are associated with $1200 \mu \mathrm{m}$ cores. There are clearly considerable differences between the star forming regions north of the ONC (OMC 2/3), and those directly south (OMC 4/5).

As we move further south (through L 1641-N towards L 1641-C, see Fig. 1) fewer protostars are identified in the Spitzer analysis. Even so, many sources are clearly driving $\mathrm{H}_{2}$ flows: in many regions (e.g. around L 1641-N, HH 34 and HH 1/2 in Figs. 6-8) we note a clear association between Spitzer protostars, (sub)millimetre cores and $\mathrm{H}_{2}$ outflows.

The infrared jets and outflows in the very busy region around L 1641-N (Fig. 6) are described in detail by Gålfalk \& Olofsson (2007) and Stanke \& Williams (2007). The former present Spitzer observations and PMs for knots close to L 1641-N itself; the latter show high-resolution $\mathrm{CO}$ maps that reveal a number of bipolar outflows centred on this cluster. The HH objects in the region are discussed by Reipurth et al. (1998). Sta02 identify seven
$\mathrm{H}_{2}$ flows in and around L 1641-N (SMZ 48-SMZ 54); we label two additional flows, DFS 118 - which is associated with a bipolar CO outflow (Stanke \& Williams 2007), and DFS 115 - which is associated with a small cluster of protostars $\sim 3^{\prime}$ south of L 1641-N, known as Strom 11. Six of these nine $\mathrm{H}_{2}$ flows have candidate Spitzer protostellar sources; all but one of these protostars is associated with $1200 \mu \mathrm{m}$ emission or a MAMBO core. In total there are 10 protostars within a $5^{\prime}$ radius of L $1641-\mathrm{N}$; it seems likely that most, if not all of these sources are associated with $\mathrm{H}_{2}$ line emission features.

The region to the west of L 1641-N, around HH 34, is shown in Fig. 7. The source of the well-known HH 34 jet, HH 34-IRS (IRS 55), is flagged as a protostar in the Spitzer data, along with two other sources, one of which (IRS 56) drives a second $\mathrm{H}_{2}$ flow. North of HH 34, the well-known HH objects HH 33/40 have in recent years been considered to be part of a parsec-scale flow that includes HH 34 and HH 86/87/88 to the south (Devine et al. 1997; Eislöffel \& Mundt 1997). However, in the Spitzer analysis a protostar (IRS 116) is found coincident with HH 40. 


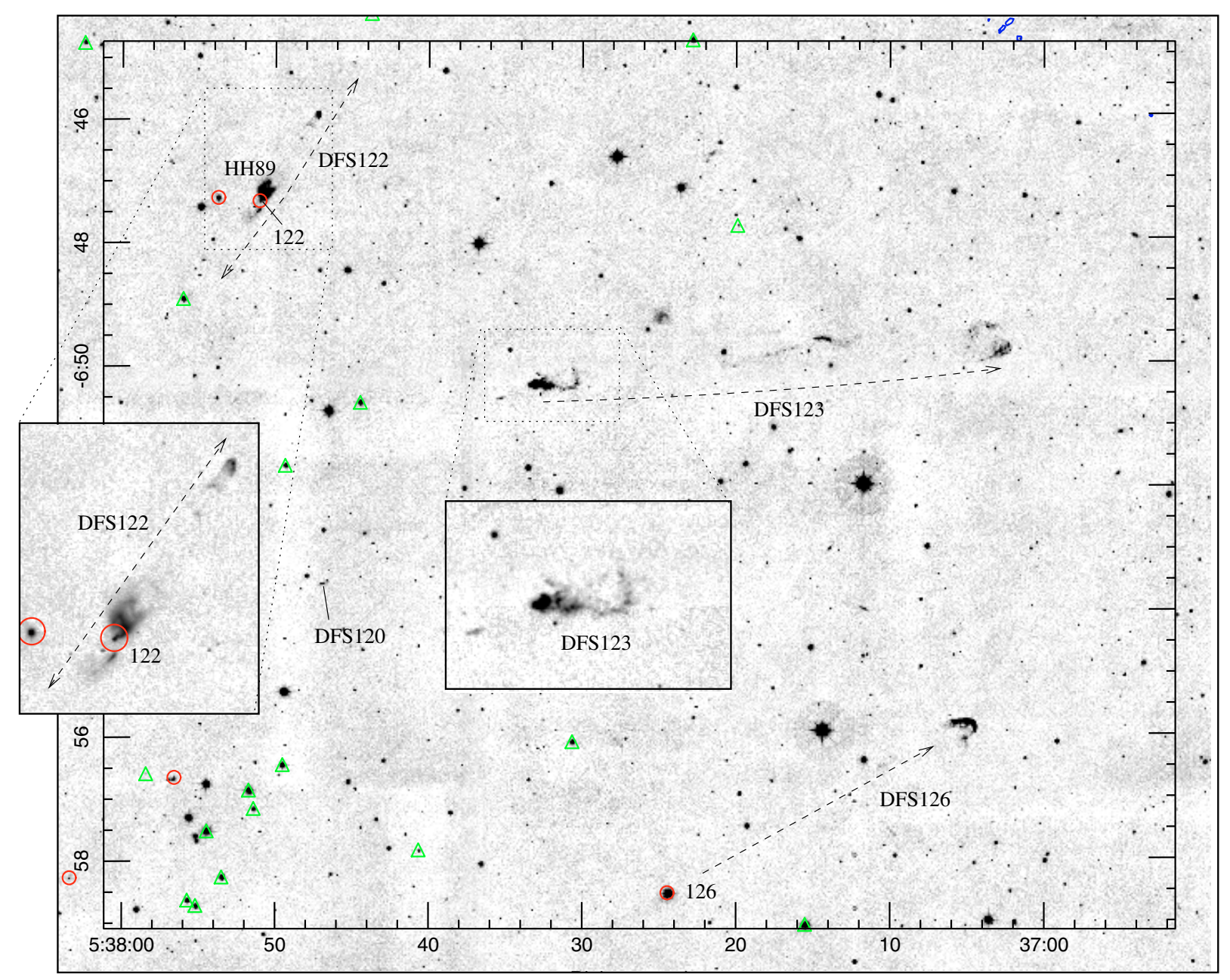

Fig. 9. Narrow-band $\mathrm{H}_{2} 2.122 \mu \mathrm{m}$ images of the region $\sim 0.5^{\circ}$ east of $\mathrm{HH} 1 / 2$ in NGC 1999 (see Fig. 1). Both insets are displayed at double-scale and with a logarithmic stretch. Note that the MAMBO $1200 \mu \mathrm{m}$ (and SCUBA $850 \mu \mathrm{m}$ ) maps do not cover this region. Symbols are as in Fig. 3.

The source is point-like in $24 \mu \mathrm{m}$ MIPS data so is probably not a mis-identified $\mathrm{H}_{2}$ feature ${ }^{2}$. IRS 116 is not associated with a dense SCUBA or MAMBO core. Even so, it remains a viable candidate outflow source for HH 33/40.

Roughly $25^{\prime}$ west of HH 34, HH 83/84 (Reipurth 1989) is also observed in $\mathrm{H}_{2}$ emission. $\mathrm{HH}$ 83-IRS, the source of this well-known jet, is outside the bounds of the SCUBA and MAMBO observations and the Spitzer IRAC $3.6 \mu \mathrm{m}$ and $5.8 \mu \mathrm{m}$ and MIPS $24 \mu \mathrm{m}$ maps, although a bright source is detected in the remaining IRAC bands with an infrared excess. HH 83-IRS's [4.5]-[8.0] colour is more consistent with a disc-excess source than a protostar.

The outflows in the vicinity of HH 1/2, shown here in Fig. 8, are well documented (see e.g. Davis et al. 1994; Hester et al. 1998; Reipurth et al. 2000). Radial velocities and PMs - based on optical observations - have been reported in the literature for the central bright HH flows (Eislöffel et al. 1994; Bally et al. 2002), so we have only measured PMs for the bright $\mathrm{H}_{2}$ features $4^{\prime}-5^{\prime}$ northeast of $\mathrm{HH} 1 / 2$. We find that these knots are moving southward, which confirms their proposed association with

\footnotetext{
${ }^{2} \mathrm{H}_{2}$ features produce pure-rotational emission lines in each of the four Spitzer IRAC bands, though not in the MIPS $24 \mu \mathrm{m}$ band (e.g. Smith \& Rosen 2005; Velusamy et al. 2007). Spectral indices measured from the IRAC bands alone can therefore mimic values expected for protostars (Davis et al. 2008), though a MIPS detection would then require bright, forbidden line emission from [Fe II].
}

the large-scale SMZ 49 outflow. Around HH 1/2 itself, only four Spitzer protostars are identified (SMZ 62/HH 3 is probably a mis-identified $\mathrm{H}_{2} \mathrm{knot}$ ). Three of the four protostars are associated with $1200 \mu \mathrm{m}$ cores; all four sources likely drive $\mathrm{H}_{2}$ flows. The well-known VLA source of the HH 1/2 outflow is unresolved from neighbouring sources and therefore is not listed as an "IRS" source in Table 2.

By extending the maps to the southeast and southwest of the HH $1 / 2$ region we discover six more $\mathrm{H}_{2}$ flows, DFS 120 and DFS 122-DFS 126, four of which are shown in Fig. 9 (DFS 122 is associated with HH 89; Reipurth 1985). Three of the six have candidate protostellar sources (one was not observed with Spitzer). With a length of $1.1 \mathrm{pc}$, DFS 123 qualifies as a "parsecscale" flow.

Roughly $30^{\prime}$ to the southeast of HH $1 / 2$, the Spitzer data recover the cluster of young stars collectively known as L 1641-C (Fig. 10). However, like Sta02 we identify only one clear-cut flow in the region, SMZ 66. The cluster is associated with only faint $1200 \mu \mathrm{m}$ emission and so L 1641-C probably harbours a fairly evolved population. The absence of ambient material explains the lack of $\mathrm{H}_{2}$ flows.

Figure 11 shows the parsec-scale flow $\mathrm{HH}$ 38/43/64 (SMZ 67). The source of this outflow, and the neighbouring $\mathrm{H}_{2}$ jet SMZ 68, are both retrieved from the Spitzer data. These protostars are also associated with molecular cores. If we include 


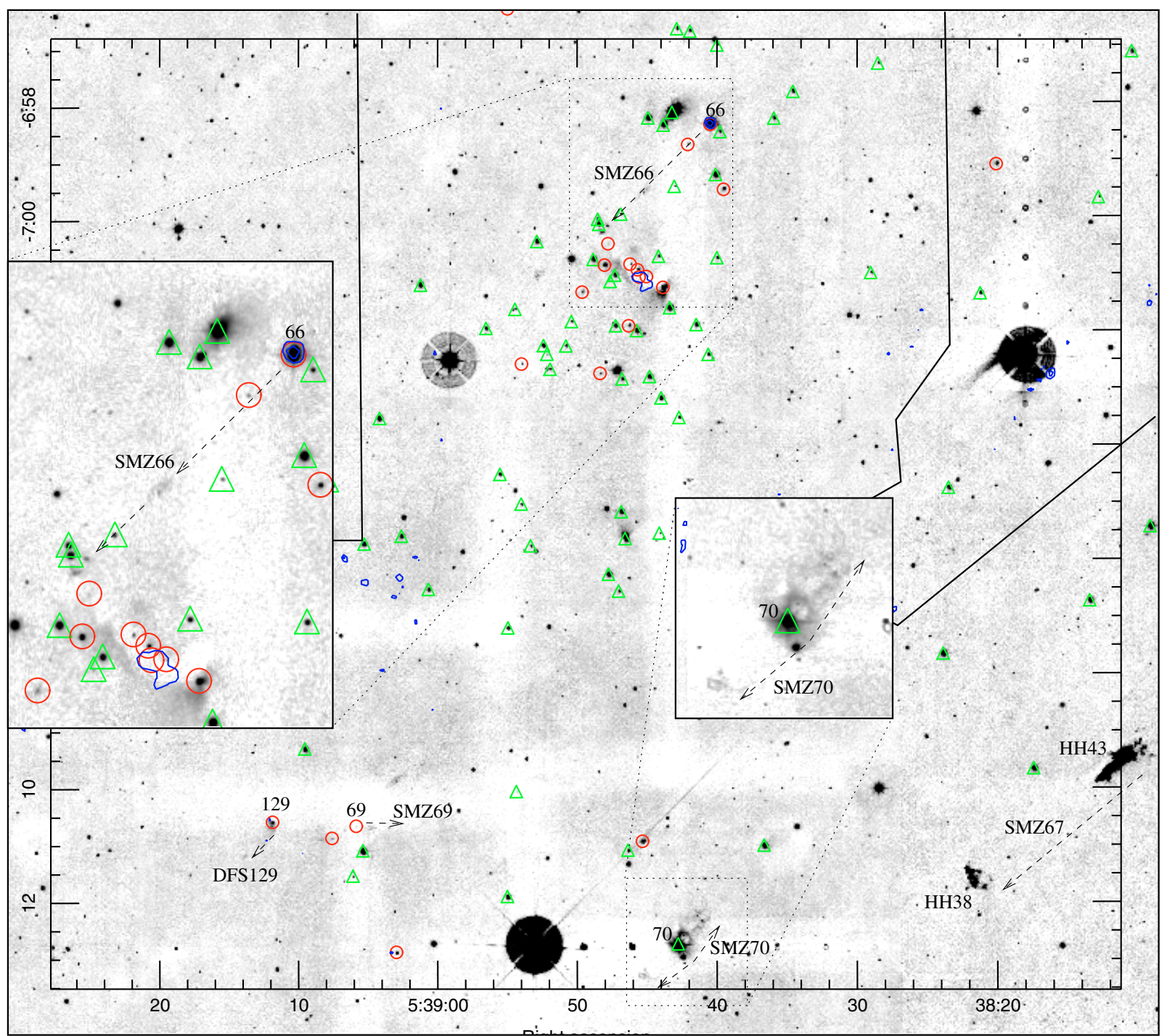

Fig. 10. Narrow-band $\mathrm{H}_{2} 2.122 \mu \mathrm{m}$ images of L 1641-C (labelled in Fig. 1). Both insets are displayed with a logarithmic scale and at twice the scale of the main figure. The northeast and northwest edges of the MAMBO map are marked with full lines. Contours and symbols are as in Fig. 3.

the DFS 126 bow-shock in the HH 38/43/64 outflow, then the total length of this system is $25^{\prime}(3.2 \mathrm{pc})$.

The region midway between L 1641-C and L 1641-S (Fig. 12; see also Fig. 1 for reference) includes the well-known T Tauri star Haro 4-255 (Aspin \& Reipurth 2000), its infrared neighbour Haro 4-255 FIR, and the spectacular arc of $\mathrm{H}_{2}$ emission, SMZ 76, first noted by Stanke (2000). SMZ 74 (not shown here), SMZ 75 and SMZ 76 all have morphologies and PMs that imply motions away from the chain of young stars near the centre of Fig. 12, although precise associations are difficult to make. Stanke (2000) identified a millimetre peak southwest of SMZ 76 (which he labelled L 1641-S3 MMS1) as the location of the likely source of this object; we note that DFS 132 may be the counter-lobe of this remarkable outflow. The protostar/dust core IRS 131/MMS 131 may also drive an $\mathrm{H}_{2}$ flow (DFS 131), though there are perhaps a dozen Spitzer YSOs in this region with no obvious $\mathrm{H}_{2}$ jet. Most have no associated dust core and are therefore, like the young stars in L 1641-C, probably too evolved.

Lastly, the region at the southern end of L 1641 is abundant with Spitzer-identified protostars and newly discovered $\mathrm{H}_{2}$ flows. In Fig. 13 we label six possible outflows, four of which have candidate sources (note that the MAMBO observations do not extend this far south in L 1641). DFS 138 is the most spectacular $\mathrm{H}_{2}$ flow, comprising a bright, collimated, bipolar jet and sweeping bow shocks. The flow extends over $13.4^{\prime}(1.8 \mathrm{pc})$ and is probably driven by the Spitzer protostar labelled IRS 138.

\subsection{Outflow statistics}

In Tables 2 and 3 we list 116 jets, of which 43 are newly identified. Of the 111 flows that were within the bounds of the Spitzer observations, 72 (62\%) have catalogued Spitzer protostellar sources: a further 12 flows are tentatively associated with Spitzer sources (IRS source numbers marked with a question mark in Tables 2 and 3). Two well-defined flows, HH 1/2 and V380 Ori-NE, have established sources that were not flagged as young stars in the analysis of the Spitzer data (the former because it is too close to a neighbouring source, the latter because it is too faint); a third object, $\mathrm{HH} \mathrm{83,} \mathrm{was} \mathrm{only} \mathrm{observed} \mathrm{in} \mathrm{two} \mathrm{of}$ the four Spitzer IRAC bands. Closer examination of the mid-IR data shows that HH 1/2 and V380 Ori-NE are probably driven by protostars, and $\mathrm{HH} 83$ by a disc-excess source. 


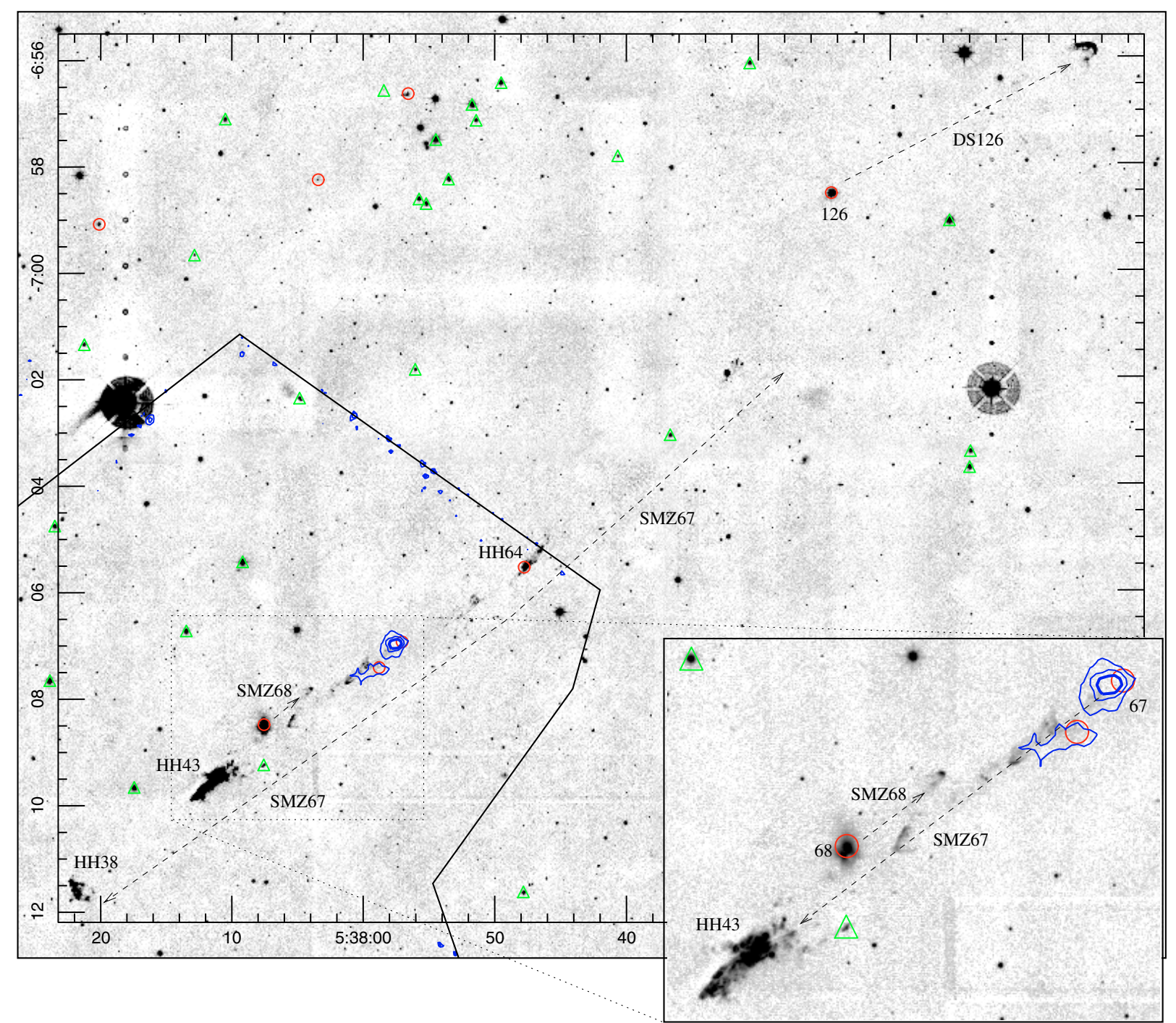

Fig. 11. Narrow-band $\mathrm{H}_{2} 2.122 \mu \mathrm{m}$ images of the region $\sim 0.3^{\circ}$ west-southwest of the $\mathrm{L} 1641-\mathrm{C}$ cluster that includes the extensive $\mathrm{HH} 38 / 43 / 64$ bipolar outflow (see Fig. 1). HH 43 and its source are shown inset at double-scale with a log stretch. The northern and western edges of the MAMBO map are marked with a full line. Contours and symbols are as in Fig. 3.

In total, $97 \mathrm{H}_{2}$ outflows (including all of the SMZ jets) were observed with MAMBO at $1200 \mu \mathrm{m} .53(55 \%)$ are associated with MAMBO (MMS) cores and young Spitzer sources; a further four outflow sources are associated with extended $1200 \mu \mathrm{m}$ emission or are unresolved from brighter, nearby cores.

Of the 116 jets, 68 (59\%) exceed an arcminute in length (at a distance of $450 \mathrm{pc}, 1^{\prime}$ is equivalent to $0.13 \mathrm{pc}$ ); 10 are parsecscale jets, exceeding 7.6' (SMZ sources 6, 49, 55, 67, 76 and the newly-discovered DFS flows 117, 123, 132, 133 and 138). Throughout Orion A we measure a range of jet lengths (as traced in $\mathrm{H}_{2}$ ); a histogram showing the distribution of lengths, uncorrected for inclination angle, shows that the number of flows with a given length decreases more-or-less exponentially (Fig. 14a). The same trend is seen if we just consider flows north of the ONC or flows south of the ONC: the map in Fig. 15 shows that the distribution of short $\left(L<1^{\prime}\right)$, medium-length, and long $\left(L>2^{\prime}\right)$ jets is more-or-less even across Orion A.

The jet lengths in Tables 2 and 3 are of course lower limits, since they are uncorrected for inclination to the line of sight. Also, as a jet or wind exits its molecular cloud, it will no longer entrain and shock-excite molecules and may not be visible in $\mathrm{H}_{2}$ emission. The histograms in Fig. 14a are therefore biased towards shorter flow lengths. Even so, if all of the jets were of equal length and randomly inclined with respect to the line of sight, inclination alone would produce a histogram that increases towards longer jet lengths (peaking at an angle of $\pi / 2$, neglecting any selection effects due to detectability as a function of angle). We can therefore be sure that the " $\mathrm{H}_{2}$ flows" in Orion are mostly short; macro-jets are a rare occurrence and, statistically at least, $\mathrm{H}_{2}$ jet lengths do not appear to be dictated by the changing environment in Orion A.

We find no real correlation between $\mathrm{H}_{2}$ jet length and indicators of the outflow source age, such as the spectral index, $\alpha$ (defined in Sect. 2.3), the integrated $1200 \mu \mathrm{m}$ flux of the core associated with each outflow source, $I_{1200}$, or the $1200 \mu \mathrm{m}$ surface brightness measured towards each outflow source, $F_{1200}$ (Fig. 16 - note that $F_{1200}$ is not always the flux density measured towards the core peak). Five of the six longest $\mathrm{H}_{2}$ flows are associated with IRS sources with large spectral indices $(\alpha>1.5)$, though at the same time four of these five protostars seem to be associated with modest-sized cores $\left(I_{1200}<1.5 \mathrm{Jy} ; F_{1200}<\right.$ $0.6 \mathrm{Jy})$ - the fifth protostar was not observed with MAMBO. The longest $\mathrm{H}_{2}$ jet in Orion A, SMZ $49\left(L \sim 35^{\prime}\right)$, is almost certainly driven by a source in the compact L 1641-N cluster, 


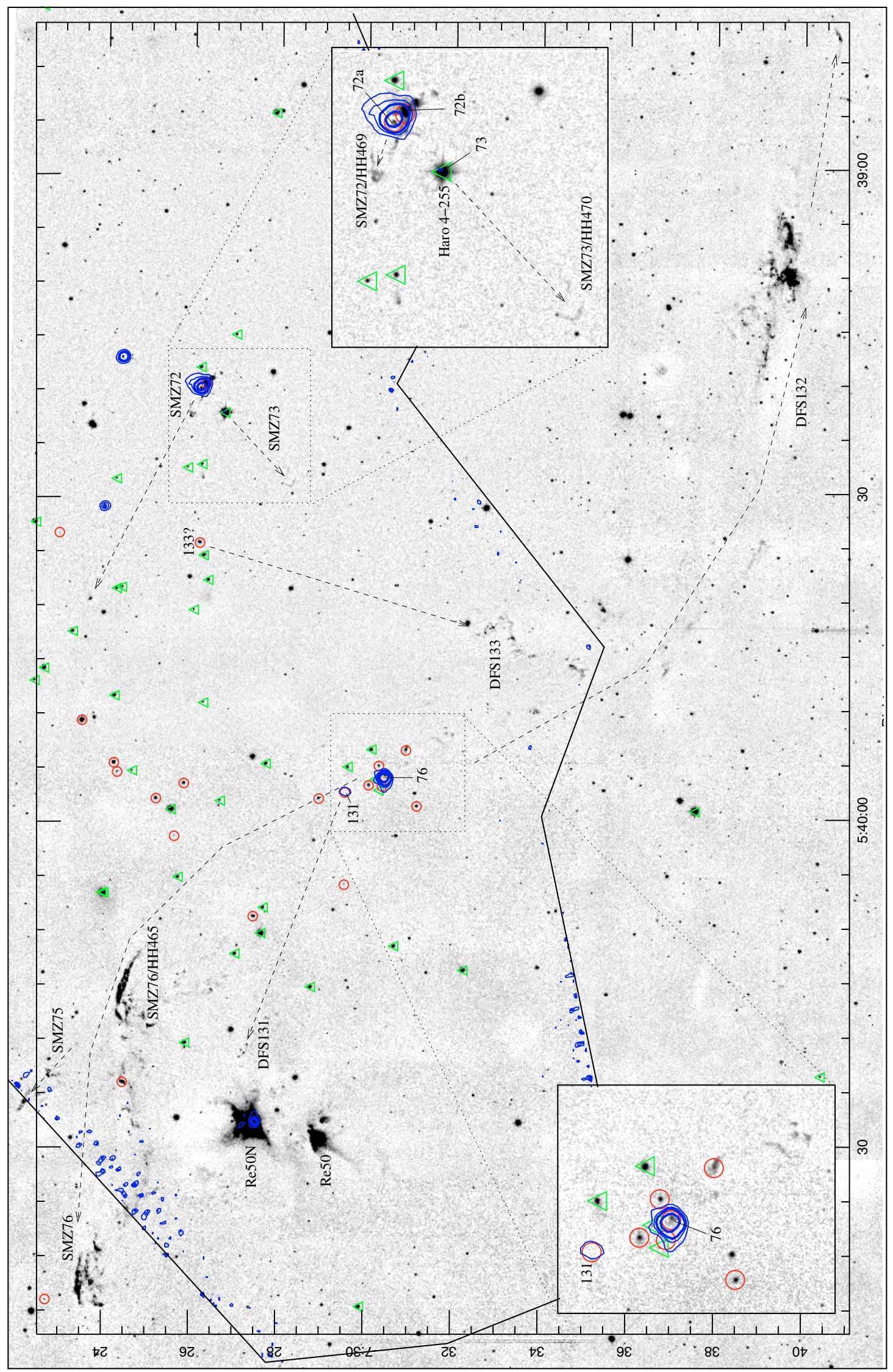

Fig. 12. Narrow-band $\mathrm{H}_{2} 2.122 \mu \mathrm{m}$ images of the region around Re 50 and Haro 4-255, midway between L 1641-C and L 1641-S in Fig. 1. The figures inset show the regions around Haro 4-255 and the likely source of SMZ 76 (both displayed with a log stretch/double size). The southern edges of the MAMBO map are marked with a full line. Contours and symbols are as in Fig. 3. 


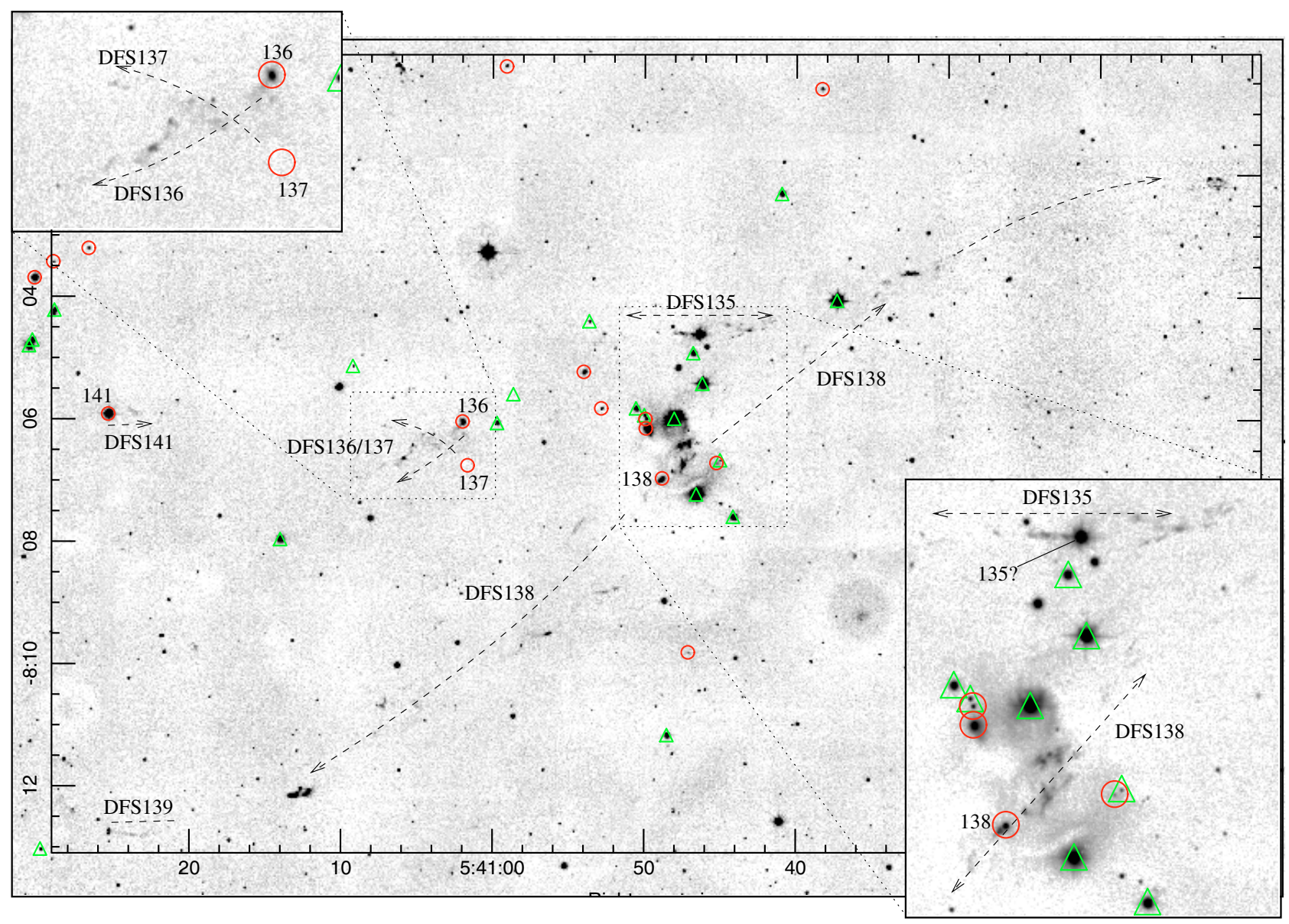

Fig. 13. Narrow-band $\mathrm{H}_{2} 2.122 \mu \mathrm{m}$ images of L 1641-S, at the bottom of the overview mosaics in Fig. 1. No MAMBO (or SCUBA) observations are available for this region. The figures shown inset are displayed at twice the scale and with a log stretch. Symbols are as in Fig. 3.

which is associated with highly-reddened protostars and dense, massive molecular cores. However, identifying this remarkable jet's progenitor and, in particular, resolving the associated core, is difficult.

There is some indication from millimetre observations of $\mathrm{CO}$ outflows that Class 0 sources produce more collimated flows than their Class I counterparts (Lee et al. 2002; Arce \& Sargent 2006). This may be due to the destruction of the protostellar core by the outflow itself, as it entrains and ploughs away the ambient gas. Since $\mathrm{H}_{2}$ emission traces the interaction of outflows with the surrounding molecular gas, one might expect to see some indication of this "flow broadening" in $\mathrm{H}_{2}$ images of outflows from sources with lower values of $\alpha$, or low values of $I_{1200}$. However, this does not seem to be the case: the flow opening angle, defined as a cone that encloses all $\mathrm{H}_{2}$ emission line features, with an apex centred on the outflow source, appears to be unrelated to $\alpha, I_{1200}$ or $F_{1200}$ (Fig. 16).

If we simply compare sources associated with a molecular core with sources undetected in $1200 \mu \mathrm{m}$ emission, we again find that both samples essentially have the same distributions of $\mathrm{H}_{2}$ jet lengths and jet opening angles: for $\mathrm{H}_{2}$ jet sources with cores the mean length is $3.9^{\prime}$ ( standard deviation $=5.6^{\prime}$; range $\left.=0.2^{\prime}-34^{\prime}\right)$; for those without cores the mean length is $1.7^{\prime}\left(\right.$ std dev $=1.9^{\prime} ;$ range $\left.=0.1^{\prime}-9.5^{\prime}\right)$. The former is skewed somewhat by the fact that four of the seven longest $\mathrm{H}_{2}$ flows $\left(L>10^{\prime}\right)$ are associated with IRS sources that do coincide with $1200 \mu \mathrm{m}$ cores (the other three long flows were not observed at $1200 \mu \mathrm{m}$ ). The mean opening angles for $\mathrm{H}_{2}$ jets with and without cores are $27.7^{\circ}\left(\right.$ std dev $=18^{\circ}$; range $\left.=4^{\circ}-70^{\circ}\right)$ and $19.9^{\circ}\left(\operatorname{std} \operatorname{dev}=15^{\circ} ;\right.$ range $\left.=3^{\circ}-50^{\circ}\right)$, respectively.

The lack of a correlation between $\alpha$ and $\mathrm{H}_{2}$ jet length, $L$, or opening angle, $\theta$, may be a result of the fact that $\alpha$ varies with orientation as well as source "age" (e.g. Whitney et al. 2003). More evolved sources driving the longest jets are expected to have lower spectral indices. However, jets inclined towards the observer will appear shorter and will likewise have lower spectral indices. Poor resolution and source confusion may also explain the lack of a correlation between core parameters $\left(I_{1200}\right.$ and $\left.F_{1200}\right)$ and $L$ or $\theta$. One should also note that $\mathrm{H}_{2}$ is not always an ideal measure of flow parameters. As noted earlier, $\mathrm{H}_{2}$ excitation only occurs when the flow impacts dense ambient material, and pre-existing $\mathrm{H}_{2}$ features cool and fade very rapidly (within a few years). For a flow that exits a molecular cloud, $\mathrm{H}_{2}$ emission features will not be observed, so the true jet length may be underestimated. Conversely, jet opening angles may be over-estimated: the wings of jet-driven bow shocks are often much wider than the underlying jet (SMZ 42 is a nice example of this in Orion), and changes in flow direction due to precession will, over time, increase the apparent opening angle.

Finally, the precise relationship between $\alpha$ and source age has not yet been established. Spectral typing of protostars is difficult because of extinction and veiling of photospheric absorption lines (Greene \& Lada 2002; Doppmann et al. 2003; Beck 2007; Antoniucci et al. 2008), so age estimates based on the fitting of theoretical mass tracks and isocrones, especially for the youngest sources, can be problematic (see e.g. 

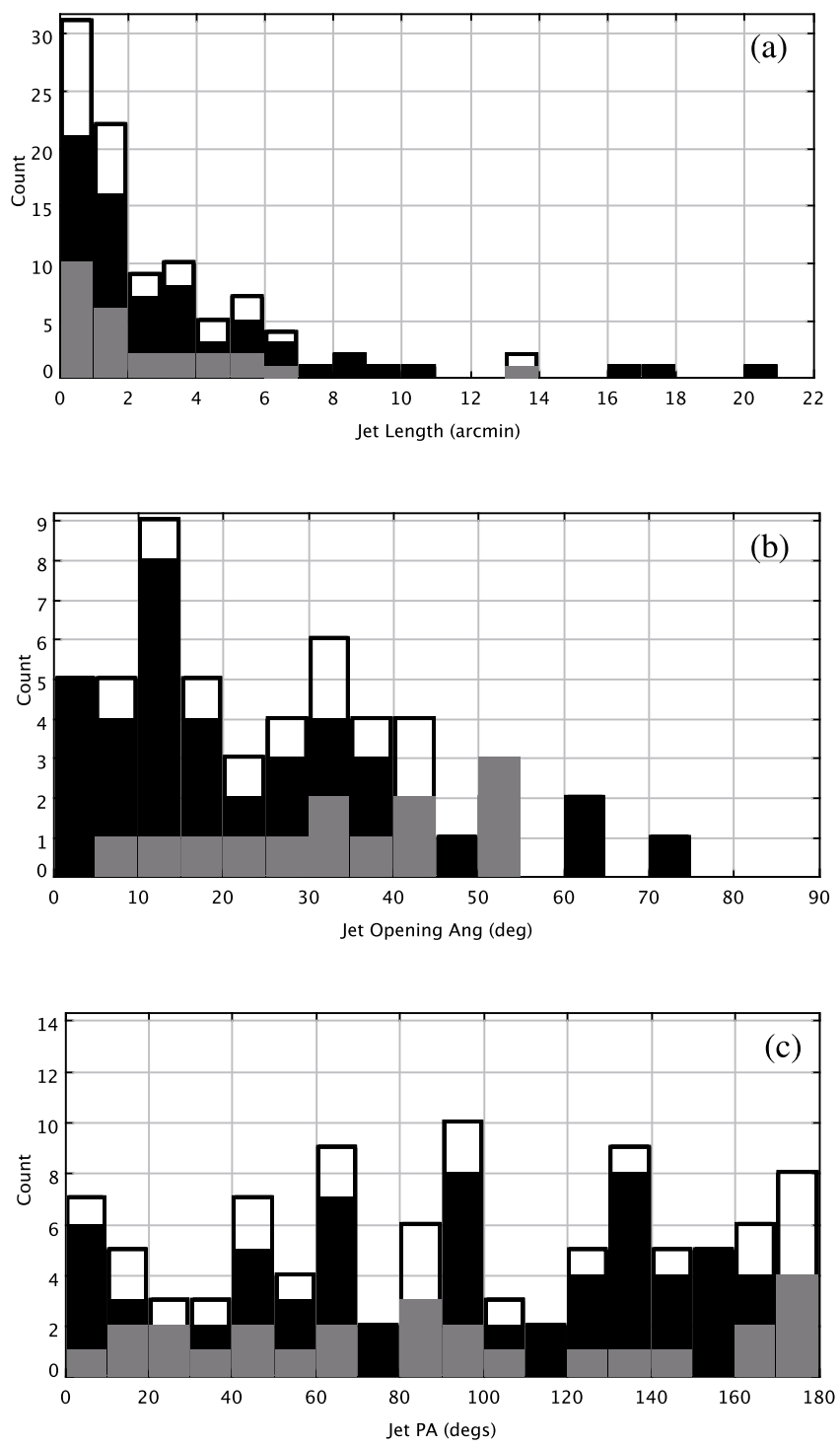

Fig. 14. Histograms showing a) the distribution of jet lengths (in $1^{\prime}$ bins), b) jet opening angles ( $5^{\circ}$ bins), and c) jet position angles ( $10^{\circ}$ bins), for all $\mathrm{H}_{2}$ jets in Orion $\mathrm{A}$ (open columns), for $\mathrm{H}_{2}$ flows north of the $\mathrm{ONC}$ (declination $>-5^{\circ} 30^{\prime}$; grey filled columns) and for $\mathrm{H}_{2}$ flows south of the ONC $\left(<-5^{\circ} 30^{\prime}\right.$; black filled columns).

Doppmann et al. 2005). Radio continuum observations may be a better way to quantify the youth of an outflow source.

\subsection{Flow orientations}

$\mathrm{H}_{2}$ jet position angles on the sky should be well defined, provided the outflow source is correctly identified. $\mathrm{H}_{2}$ surveys can therefore be used to test the standard paradigm that clouds collapse along field lines to form elongated, clumpy filaments from which chains of protostars are born (Mouschovias 1976; Larry et al. 2008), with the associated outflows aligned parallel with the local field, and perpendicular to the chains of cores (Banerjee $\&$ Pudritz 2006). The presence of magnetic fields in molecular clouds has been demonstrated, and the orientations of field lines with respect to chains of oblate cores have been measured in a number of star forming regions (e.g. Crutcher et al. 1999; Matthews et al. 2002; Houde et al. 2004; Vallée \& Fiege 2006; Larry et al. 2008). In a few regions there is also some indication that outflows are preferentially orientated orthogonal to

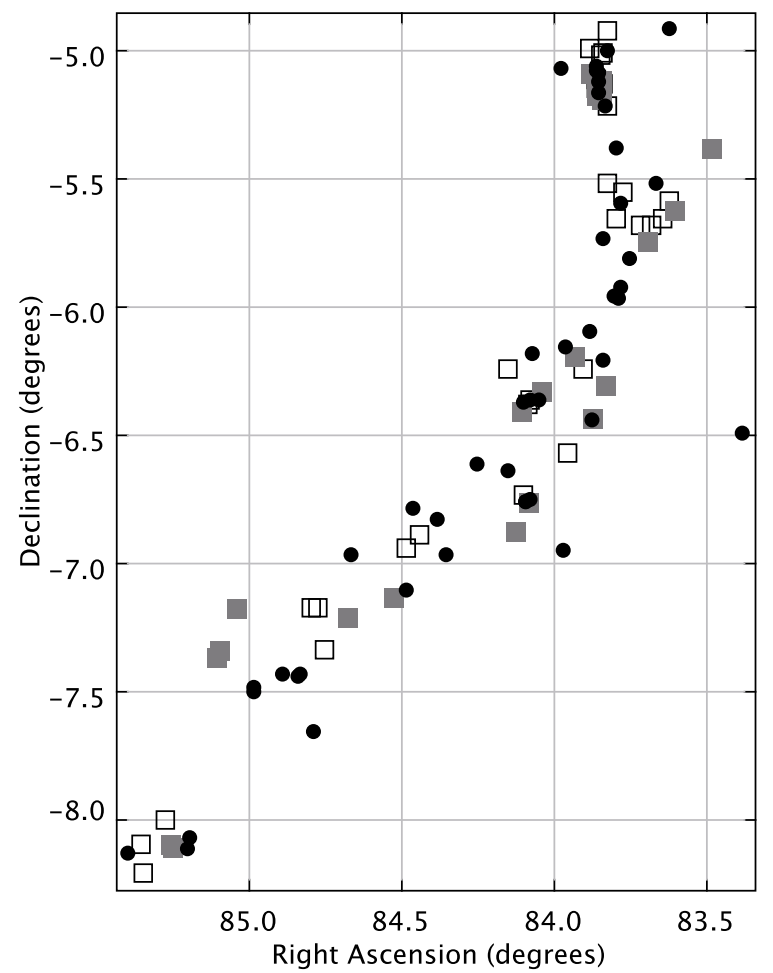

Fig. 15. A map showing the distribution of short ( $L<1^{\prime}$; open squares), intermediate $\left(1^{\prime}<L<2^{\prime}\right.$; filled grey squares), and long ( $L>2^{\prime}$; filled black circles) $\mathrm{H}_{2}$ jets.

individual cores or chains of cores, and/or parallel with the local magnetic field (Strom et al. 1986; Heyer et al. 1987; Ménard \& Duchene 2004; Davis et al. 2007; Ananthapindika \& Whitworth 2008).

The Orion A GMC comprises a large number of cores extended along a north-south "integral-shaped" filament that passes through the Orion nebula and extends southward, broadening out between L 1641-N and L 1641-S (Johnstone \& Bally 1999, 2006; Nutter \& Ward-Thompson 2007). It is therefore potentially an ideal laboratory for an investigation into the relationship between cores, filaments and outflows. Throughout much of Orion A, one might expect to see flows predominantly orientated east-west.

In Fig. 14c we have plotted a histogram of the jet position angles (in ten degree bins) for all outflows in our sample. The plot suggests a uniform distribution of position angles. Moreover, a Kolmogorov-Smirnov (KS) test shows that there is a $>99 \%$ probability that such a distribution is drawn from a homogeneously distributed sample. In other words, the position angles of the $\mathrm{H}_{2}$ flows are completely random. If we consider only the jets with identified outflow sources a similar situation is found. In this case the probability that the distribution is random is $99.2 \%$. The same also holds if we separate the $\mathrm{H}_{2}$ jets in the northern region $\left(\mathrm{Dec}>-6^{\circ}\right.$ ), where the dense gas is more tightly constrained to a north-south filament, from those south of the ONC $\left(\right.$ Dec $\left.<-6^{\circ}\right)$. Even for the compact ridge of pre- and proto-stellar cores north of the ONC (OMC 2/3), the outflows are randomly orientated. Similar plots for $\mathrm{H}_{2}$ jets exceeding an arcminute in length (flows that arguably have more reliable position angles), or for jets with embedded protostars coincident with MAMBO cores, are equally randomly orientated.

So the $\mathrm{H}_{2}$ jets in Orion A appear to be randomly orientated. Notably, Matthews et al. (2001) and Houde et al. (2004) find 

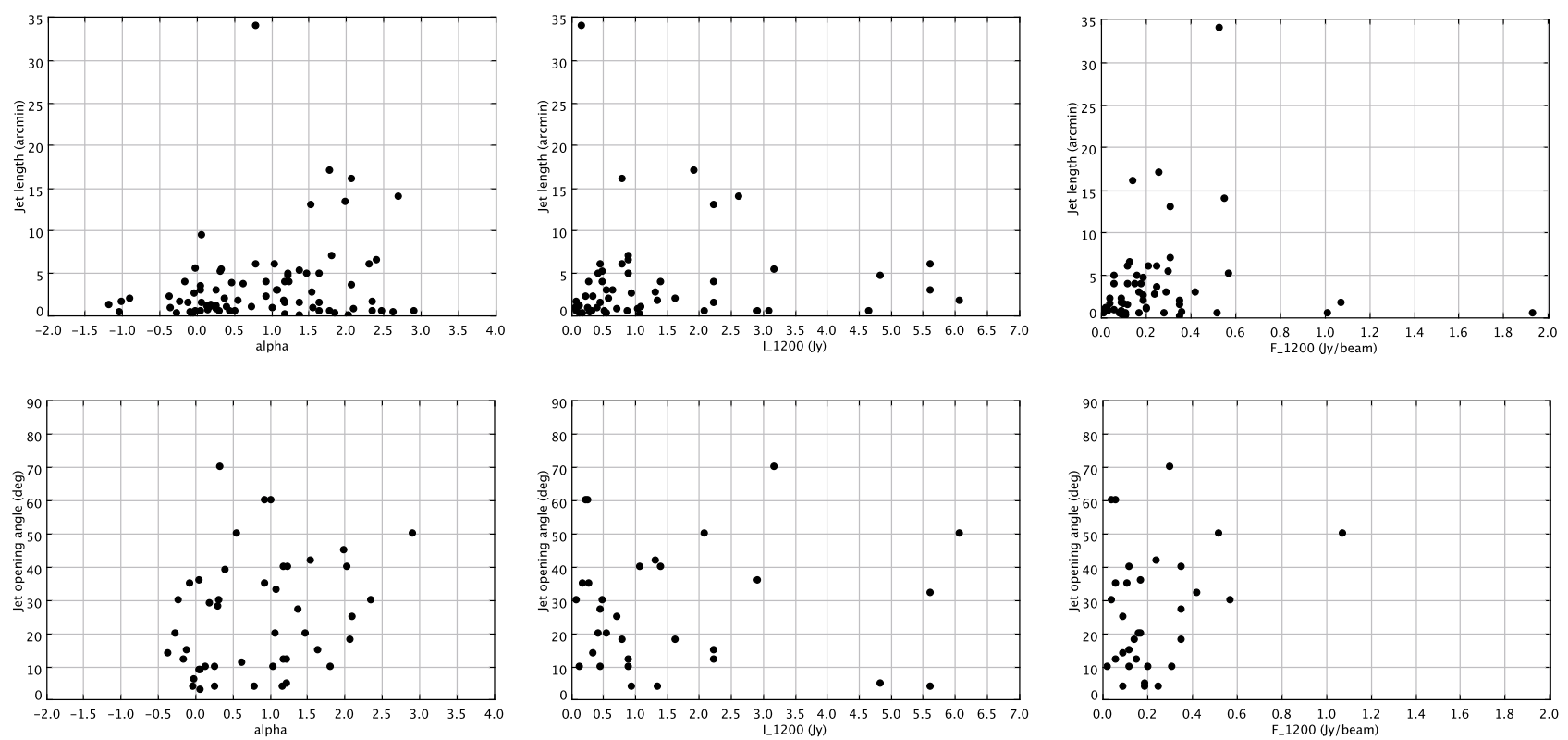

Fig. 16. Source spectral index, $\alpha$, integrated $1200 \mu \mathrm{m}$ core flux, $I_{1200}$, and $1200 \mu \mathrm{m}$ flux density, $F_{1200}$, plotted against $\mathrm{H}_{2}$ jet length and jet opening angle for all jets in Orion A with identified sources (Tables 2 and 3).

that on large scales the field along the integral-shaped filament (north and south of the ONC) is non-uniform. The long axes of the elongated cores along the filament also appear to be randomly orientated (Chini et al. 1997; Johnstone \& Bally 1999, 2006; Nutter \& Ward-Thompson 2007; see also e.g. Fig. 3). It is therefore not too surprisingly that flow position angles should also be non-uniform. But are the $\mathrm{H}_{2}$ outflows orientated orthogonal to the cores associated with each outflow source? At $450 \mathrm{pc}$, the MAMBO beam of $11^{\prime \prime}$ corresponds to $5 \times 10^{3} \mathrm{AU}$, considerably larger than accretion disc dimensions or the size of the solar system. Even so, many of the cores in Tables 2 and 3 are elongated; if these "circumstellar envelopes" are magnetically tied to the accretion discs they harbour (due to modest ambipolar diffusion), then one might expect them to be orientated perpendicular to the flow axes (Banerjee \& Pudritz 2006; Ananthapindika \& Whitworth 2008).

In our data, $47 \mathrm{H}_{2}$ jet sources are associated with $1200 \mu \mathrm{m}$ emission above a surface density $F_{1200}>75 \mathrm{mJy}$. Of these, 42 are associated with cores (rather than "emission" in Tables 2 and 3). From this sample we extract sources where the core major-to-minor axis ratio exceeds 1.3 , and exclude very large (diffuse) cores where the Full Width Half Maximum of the Gaussian fit is greater than $50^{\prime \prime}$ in either axis. We arrive at a sample of just $22 \mathrm{H}_{2}$ flows with cores. Surprisingly, even from this finely-tuned sample, the distribution of $\mathrm{H}_{2}$ jet position angles (PAs) with respect to core PAs is completely random. One might expect that the "longer", more clearly-defined jets, would be orthogonal to their associated cores. However, in a plot of jet length against "jet-PA minus core-PA" (not shown), even the longest jets seem to be randomly orientated with respect to their progenitor cores.

It seems likely that, in some of the more clustered regions (OMC 2/3, L1641-N, etc.), the MAMBO observations have insufficient resolution to disentangle the molecular envelopes associated with multiple sources. Focusing on a hand-picked sample of sources separate from the main clusters of protostars, sources with well-defined jets and bright, compact cores, is probably necessary. Obviously much higher resolution (sub)mm

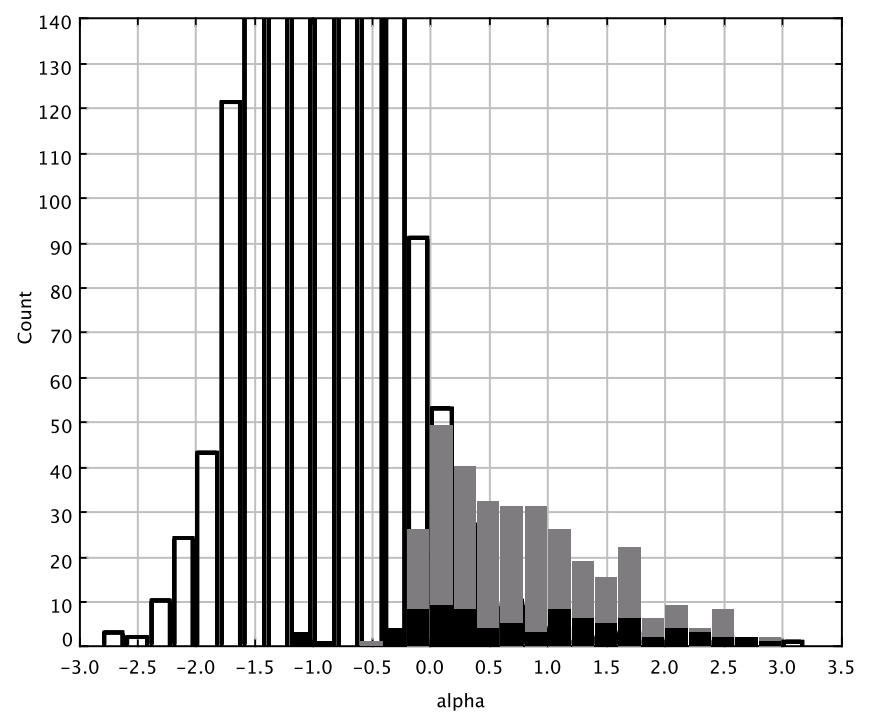

Fig. 17. Histograms showing the number of disc-excess sources (open columns), protostars (filled grey columns) and $\mathrm{H}_{2}$ flow sources (black columns) with a given value of $\alpha$ (in bins of 0.2).

observations would also be beneficial; ALMA will be useful in this respect.

\section{Discussion}

\subsection{Do all protostars drive $\mathrm{H}_{2}$ outflows?}

In Fig. 17 we plot histograms showing the distribution of spectral indices for the Spitzer disc-excess sources, the Spitzer protostars, and the outflow sources. As was found to be the case in Perseus (Davis et al. 2008) and the more distant, high mass star forming region, W 75/DR 21 (Davis et al. 2007; Kumar et al. 2007) the majority of $\mathrm{H}_{2}$ flows are driven by protostars with positive spectral indices. The mean value of $\alpha$ for the disc excess sources, most of which will be T Tauri stars, is 
-0.85 (std dev $=0.61$ ); for the protostars and the outflow sources, the mean values are $0.80($ std $\mathrm{dev}=0.72)$ and 0.86 ( std dev $=0.93$ ); in terms of spectral index, the outflow sources in Orion A appear to be indistinguishable from the protostellar sample.

For the outflow sources in Perseus and DR 21/W 75 the mean values of $\alpha$ are 1.4 and 1.9, respectively, somewhat higher than in Orion A. The protostellar population in Orion A may therefore be slightly more evolved than in Perseus and DR 21/W 75. Note, however, that the DR 21/W 75 spectral indices were derived from only IRAC photometry, which may result in a higher mean value (the Perseus indices were measured using 2MASS JHK photometry, as well as Spitzer and MIPS data). Also, the larger UV radiation field from the ONC and from B- and A-type stars spread throughout Orion A may accelerate the erosion of protostellar envelopes, thereby reducing measured values of $\alpha$, although $\alpha$ is largely defined by emission from the inner disc rather than the outer, cold envelope, so this affect may be modest.

The spectral index is clearly a useful tool when searching for $\mathrm{H}_{2}$ outflow source candidates. Yet of the 290 Spitzer-identified protostars observed in Orion A with WFCAM (excluding the dozen sources in the Orion Nebula), only $\sim 84$, or less than one third, seem to be driving outflows that excite $\mathrm{H}_{2}$ emission. Thus, at first sight it does not appear that all protostars drive $\mathrm{H}_{2}$ flows.

However, our near-IR images may not be sensitive enough to detect the weakest flows, especially $\mathrm{H}_{2}$ jets from the least luminous - and least massive - protostars (Caratti O Garatti et al. 2008). Also, the selection criteria used for identifying the Spitzer protostars may well include a handful of background galaxies and AGN ( $\sim 0.7$ per square degree), $\mathrm{H}_{2}$ emission knots, and a number of more evolved young stars. Though faint, heavily reddened Class II sources and disc-excess sources viewed edge-on can have the same colours as protostars (Whitney et al. 2003). These classical T Tauri stars rarely produce extensive $\mathrm{H}_{2}$ flows, because they have either swept away much of their circumstellar environment, or because they occupy regions with a low ambient molecular gas density (they may of course drive $\mathrm{HH}$ jets, which our near-IR survey is not sensitive to). Gutermuth et al. (2009) estimate that at most $3.6 \%$ of protostars may be edge-on discexcess sources. Our WFCAM images include 1909 disc sources (Megeath et al., in prep.). There may therefore be as many of 70 disc sources in the protostar catalogue that are too evolved to drive $\mathrm{H}_{2}$ outflows.

The fact that the distributions of $\alpha$ values for the outflow sources and protostars in Fig. 17 are comparable suggests that protostars and $\mathrm{H}_{2}$ outflow sources are essentially the same objects; as noted earlier, the spread in $\alpha$ for both samples may be due to inclination angle as much as to source age (Whitney et al. 2003; Allen et al. 2004; Megeath et al. 2004). Moreover, there is empirical evidence which suggests that the $\mathrm{H}_{2}$ outflow phase may be as long-lived as the protostellar phase: the dynamical age of an $\mathrm{H}_{2}$ flow is of the order of $10^{4}-10^{5}$ years (based on a canonical jet length of $1 \mathrm{pc}$ and molecular outflow speeds of 10-100 $\mathrm{km} \mathrm{s}^{-1}$; e.g. Arce et al. 2007), while statistical studies indicate that the low mass protostellar phase may last at least as long (Froebrich et al. 2006; Hatchell et al. 2007; Enoch et al. 2008).

Towards the end of the protostellar phase the accretion rateand therefore the mass loss rate - will evolve quite dramatically, as the source approaches the Class II T Tauri phase. Statistically at least, these sources will probably be associated with mostly atomic, low power jets, which may then persist for timescales of the order of $10^{5}-10^{6}$ years (Reipurth \& Bally 2001). Such jets may be undetectable without very deep $\mathrm{H}_{2}$ imaging, though their sources will in any case usually be identified as "disc-excess sources" in the Spitzer analysis used here.

In conclusion, it seems likely that for much of their evolution all protostars will produce outflows bright in $\mathrm{H}_{2}$ line emission. In a sample such as ours, the protostars not associated with $\mathrm{H}_{2}$ flows may be misclassified disc-excess sources, AGN or even $\mathrm{H}_{2}$ knots. Some protostars may be marginally too evolved or too low mass to produce detectable $\mathrm{H}_{2}$ jets. Orientation effects and the local, inhomogeneous environment will also play a role in jet detectability.

\subsection{Association of $\mathrm{H}_{2}$ outflow sources with molecular cores}

In a recent study, Jørgensen et al. (2007) used a combination of Spitzer IRAC and MIPS photometry and $850 \mu \mathrm{m}$ SCUBA observations to identify the youngest protostars in Perseus. They selected 49 MIPS sources that have red [3.6]-[4.5] and/or [8.0]-[24] colours, and are within $15^{\prime \prime}$ of a SCUBA peak. Davis et al. (2008) later found that most (63\% of the sources they imaged) drive $\mathrm{H}_{2}$ outflows. In Orion $\mathrm{A}$ it seems that a similarly high percentage of "protostars with cores" drive molecular $\left(\mathrm{H}_{2}\right)$ outflows; note that most of the outflows with identified progenitors in Tables 2 and 3 are associated with discrete $1200 \mu \mathrm{m}$ cores.

However, clearly not all molecular cores are associated with $\mathrm{H}_{2}$ flows. We identify in excess of 500 peaks in the $1200 \mu \mathrm{m}$ MAMBO data (Stanke et al., in prep.). Similarly, Nutter \& Ward-Thompson (2007) extract almost 400 cores from their more limited $850 \mu \mathrm{m}$ SCUBA maps of Orion A. Only about $1 / 5$ th of these cores seem to be associated with $\mathrm{H}_{2}$ outflows. (Johnstone \& Bally 2006 obtain a similar result in their comparison of SCUBA data with the $\mathrm{H}_{2}$ images of Sta02; they find that 17 of the 70 SCUBA clumps they identify are clearly associated with $\mathrm{H}_{2}$ emission.)

Many of the cores in Orion A will be prestellar-based on the number of cores without $\mathrm{H}_{2}$ jets, roughly $80 \%$ - which suggests that the $\mathrm{H}_{2}$ outflow (and therefore protostellar) phase is considerably shorter than the prestellar phase. This statistic assumes that each core produces just one protostar (and $\mathrm{H}_{2}$ flow), which is almost certainly incorrect; small groups of protostars are often found in each core (see for example the L 1641-N core in Fig. 6). The prestellar phase could therefore be at least an order of magnitude longer than the protostellar/ $\mathrm{H}_{2}$ outflow phase.

What fraction of cores containing protostars drive $\mathrm{H}_{2}$ flows? In Fig. 18 we plot the entire $1200 \mu \mathrm{m}$ map of Orion A. We overplot the positions of the Spitzer candidate protostars, and mark the locations of the $\mathrm{H}_{2}$ outflow sources. (Note that the outflow and protostar catalogues are incomplete in the Orion Nebula region; saturation effects, source confusion, and the bright, diffuse nebulosity that pervades the ONC will limit both samples.) Of the 190 protostars in Orion A that were observed with MAMBO, excluding the dozen in the Orion Nebula region, only 70 coincide with $1200 \mu \mathrm{m}$ cores (to within an $11^{\prime \prime}$ radius), although most do coincide with extended and/or diffuse emission. Of these 70 protostars with cores, $43(61 \%)$ appear to be driving molecular $\mathrm{H}_{2}$ outflows. In some of the less tightly clustered and therefore less complex regions in $\mathrm{L} 1641$, such as the NGC 1980/OMC 5 region in Fig. 5, the number of protostars associated with cores that also drive $\mathrm{H}_{2}$ jets approaches $100 \%$. Within the limitations of our millimetre and near-IR observations (discussed in the previous section), it seems likely that most, if not all protostars within molecular cores drive $\mathrm{H}_{2}$ outflows. It is also clear that the combination of Spitzer mid-IR photometry and either SCUBA $850 \mu \mathrm{m}$ or MAMBO $1200 \mu \mathrm{m}$ 


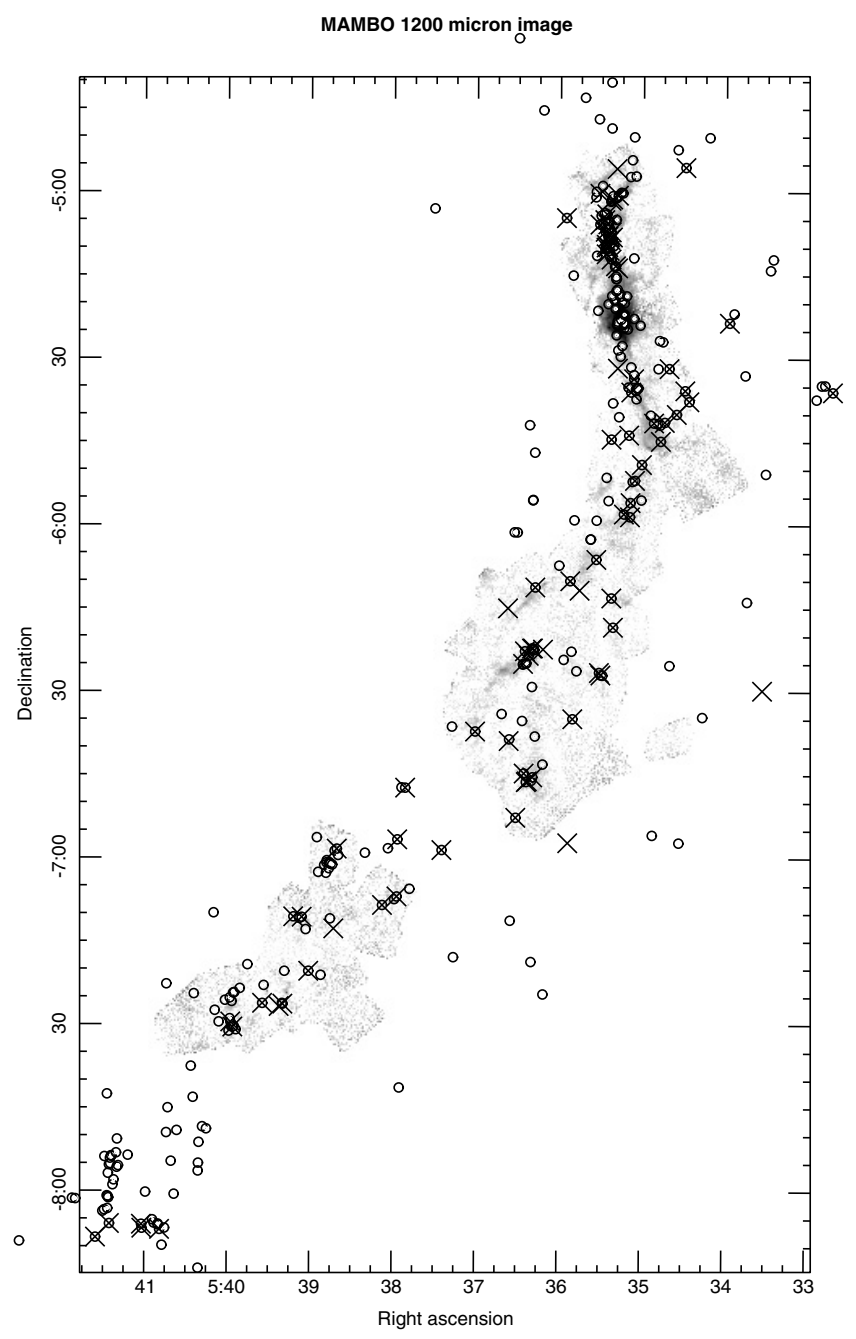

Fig. 18. A logarithmically-scaled image of $1200 \mu \mathrm{m}$ emission with, superimposed, the locations of all Spitzer-identified protostars (open circles). $\mathrm{H}_{2}$ outflow sources are marked with crosses (note that a handful of $\mathrm{H}_{2}$ jet sources are disc-excess sources).

far-IR photometry is ideal for identifying molecular outflow sources and, in all probability, the youngest protostars.

Lastly, we mention that, of the 30 or so protostars in Fig. 18 that are located to the east or west of the edges of the MAMBO map, i.e. beyond the bounds of the high-density molecular ridge that constitutes OMC 2/3 and L 1641, only three protostars are driving $\mathrm{H}_{2}$ flows. A much larger fraction of protostars within the bounds of the MAMBO map, where ambient gas densities are much higher, are driving molecular outflows. As was noted in the previous section, some of the protostars without $\mathrm{H}_{2}$ jets may be marginally more evolved; Fig. 18 suggests that these "evolved protostars", sources close to the Class II T Tauri phase (which may of course drive $\mathrm{HH}$ jets), are more widely distributed.

\subsection{Clustering and the distribution of protostars and outflow sources}

Qualitatively speaking, if one examines Fig. 18 closely, it appears that clusters of protostars are not always associated with clusters of $\mathrm{H}_{2}$ outflows. In L 1641-C, for example (RA $5^{\mathrm{h}} 38.7^{\mathrm{m}}$, Dec $\sim 7^{\circ} 00^{\prime}$; see also Fig. 10), although a dozen protostars and $\sim 30$ disc-excess sources are identified, there is only one $\mathrm{H}_{2}$ flow. Likewise, between $\mathrm{Re} 50$ and Haro 4-255
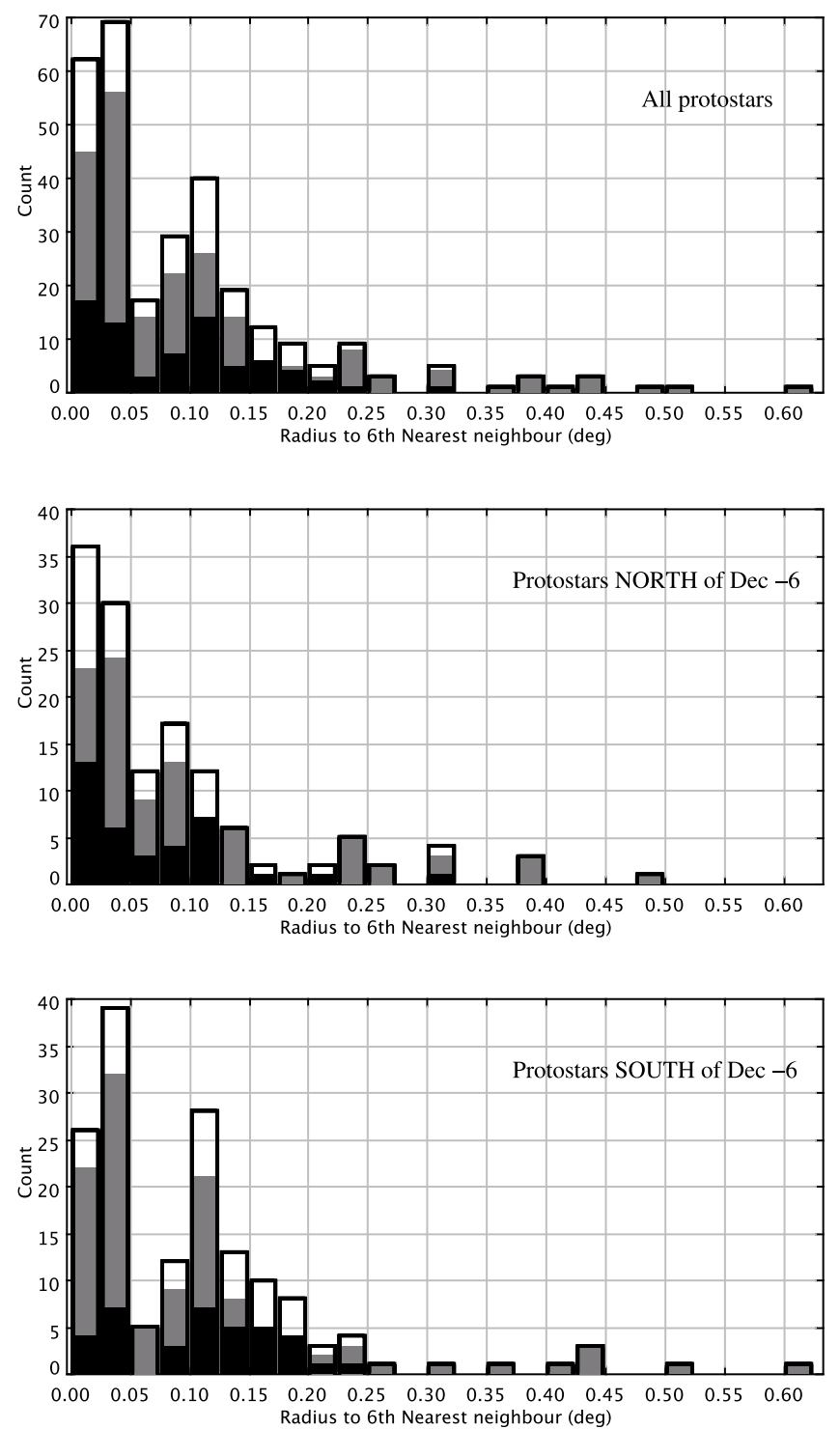

Fig. 19. Histograms showing the distribution of 6th nearest neighbour radii for all Spitzer-identified protostars (open bars), for those that drive $\mathrm{H}_{2}$ outflows (black filled bars), and for those that do not drive $\mathrm{H}_{2}$ outflows (grey filled bars). Top - entire region; middle - north of declination $-6^{\circ}$; bottom - south of declination $-6^{\circ}$.

(RA $\sim 5^{\mathrm{h}} 39.9^{\mathrm{m}}$, Dec $\sim-7^{\circ} 28^{\prime}$; see also Fig. 12) there is a chain of 16 protostars and at least as many disc-excess sources, yet again we only detect three or four molecular $\mathrm{H}_{2}$ outflows. Neither region is associated with large columns of ambient molecular material ( $\mathrm{L} 1641-\mathrm{C}$ is barely detected in the MAMBO data); the paucity of detected outflows may therefore be due to the evolved nature of the protostars in each region. L 1641-N, on the other hand, is associated with massive dense cores, and a large fraction (roughly 50\%) of the protostars power $\mathrm{H}_{2}$ outflows. Likewise, north of the $\mathrm{ONC}$, in the OMC 2/3 molecular cloud region (between declinations $-4^{\circ} 30^{\prime}$ and $-5^{\circ} 15^{\prime}$ ) where protostars and cores are very highly clustered, there are 65 protostars of which 26 appear to be driving molecular flows (this may be a lower limit, given the abundance of $\mathrm{H}_{2}$ emission line features, although protostars are also difficult to resolve in this busy, nebulous region).

We also note that regions with multiple $\mathrm{H}_{2}$ flows often seem to be more sparsely populated with protostars. For example, 
Table 1. Mean and median Nearest Neighbour (NN) radii for protostars (all sources, sources with $\mathrm{H}_{2}$ outflows, and sources without) in Orion A. The standard deviation (std) is given in brackets. North and South refer to protostars located north or south of declination $-6^{\circ}$, respectively.

\begin{tabular}{|c|c|c|c|c|c|}
\hline$\overline{\overline{R e g i o n}}$ & $\begin{array}{c}\text { 5th NN } \\
\text { mean(std)median }\end{array}$ & $\begin{array}{c}\text { 6th NN } \\
\text { mean(std)median }\end{array}$ & $\begin{array}{c}7 \text { th NN } \\
\text { mean(std)median }\end{array}$ & $\begin{array}{c}10 \text { th NN } \\
\text { mean(std)median }\end{array}$ & $\begin{array}{c}20 \text { th NN } \\
\text { mean(std)median }\end{array}$ \\
\hline All Protostars & $0.087^{\circ}\left(0.09^{\circ}\right) 0.060^{\circ}$ & $0.097^{\circ}\left(0.10^{\circ}\right) 0.070^{\circ}$ & $0.106^{\circ}\left(0.10^{\circ}\right) 0.079^{\circ}$ & $0.13^{\circ}\left(0.11^{\circ}\right) 0.11^{\circ}$ & $0.20^{\circ}\left(0.11^{\circ}\right) 0.18^{\circ}$ \\
\hline Protostars with flow & $0.077^{\circ}\left(0.06^{\circ}\right) 0.064^{\circ}$ & $0.089^{\circ}\left(0.07^{\circ}\right) 0.085^{\circ}$ & $0.098^{\circ}\left(0.07^{\circ}\right) 0.096^{\circ}$ & $0.13^{\circ}\left(0.08^{\circ}\right) 0.12^{\circ}$ & $0.19^{\circ}\left(0.10^{\circ}\right) 0.20^{\circ}$ \\
\hline Protostars no flow & $0.090^{\circ}\left(0.10^{\circ}\right) 0.055^{\circ}$ & $0.100^{\circ}\left(0.11^{\circ}\right) 0.061^{\circ}$ & $0.109^{\circ}\left(0.11^{\circ}\right) 0.073^{\circ}$ & $0.13^{\circ}\left(0.12^{\circ}\right) 0.09^{\circ}$ & $0.20^{\circ}\left(0.12^{\circ}\right) 0.17^{\circ}$ \\
\hline All Protost & $0.077^{\circ}\left(0.08^{\circ}\right) 0.042^{\circ}$ & $0.086^{\circ}\left(0.09^{\circ}\right) 0.050^{\circ}$ & $0.095^{\circ}\left(0.10^{\circ}\right) 0.058^{\circ}$ & $0.12^{\circ}\left(0.11^{\circ}\right) 0.08^{\circ}$ & $0.16^{\circ}\left(0.12^{\circ}\right) 0.14^{\circ}$ \\
\hline Protostars with flow (North) & $0.059^{\circ}\left(0.06^{\circ}\right) 0.035^{\circ}$ & $0.068^{\circ}\left(0.06^{\circ}\right) 0.048^{\circ}$ & $0.075^{\circ}\left(0.07^{\circ}\right) 0.058^{\circ}$ & $0.10^{\circ}\left(0.08^{\circ}\right) 0.11^{\circ}$ & $0.14^{\circ}\left(0.10^{\circ}\right) 0.14^{\circ}$ \\
\hline Protostars no flow (North) & $0.083^{\circ}\left(0.09^{\circ}\right) 0.047^{\circ}$ & $0.093^{\circ}\left(0.10^{\circ}\right) 0.051^{\circ}$ & $0.103^{\circ}\left(0.11^{\circ}\right) 0.059^{\circ}$ & $0.12^{\circ}\left(0.12^{\circ}\right) 0.08^{\circ}$ & $0.17^{\circ}\left(0.13^{\circ}\right) 0.13^{\circ}$ \\
\hline All Proto & $0.096^{\circ}\left(0.10^{\circ}\right) 0.075^{\circ}$ & $0.107^{\circ}\left(0.10^{\circ}\right) 0.094^{\circ}$ & $0.115^{\circ}\left(0.10^{\circ}\right) 0.101^{\circ}$ & $0.14^{\circ}\left(0.11^{\circ}\right) 0.12^{\circ}$ & $0.23^{\circ}\left(0.11^{\circ}\right) 0.21^{\circ}$ \\
\hline Protostars & $0.094^{\circ}\left(0.06^{\circ}\right) 0.093^{\circ}$ & $0.110^{\circ}\left(0.06^{\circ}\right) 0.120^{\circ}$ & $0.119^{\circ}\left(0.06^{\circ}\right) 0.137^{\circ}$ & $0.16^{\circ}\left(0.07^{\circ}\right) 0.18^{\circ}$ & $0.24^{\circ}\left(0.06^{\circ}\right) 0.23^{\circ}$ \\
\hline Protostars no flow (South) & $0.097^{\circ}\left(0.11^{\circ}\right) 0.073^{\circ}$ & $0.106^{\circ}\left(0.11^{\circ}\right) 0.079^{\circ}$ & $0.114^{\circ}\left(0.11^{\circ}\right) 0.083^{\circ}$ & $0.14^{\circ}\left(0.12^{\circ}\right) 0.11^{\circ}$ & $0.23^{\circ}\left(0.12^{\circ}\right) 0.19^{\circ}$ \\
\hline
\end{tabular}

around HH 1/2 (RA $\sim 5^{\mathrm{h}} 36.3^{\mathrm{m}}$, Dec $\sim-6^{\circ} 46^{\prime}$; see also Fig. 8) there are at least half-a-dozen $\mathrm{H}_{2}$ jets, yet only about the same number of protostars. Similarly, east of HH 1/2 around DFS 123 (Fig. 9) and further south around $\mathrm{HH} 38 / 43 / 64$ (Fig. 11) the ratio of $\mathrm{H}_{2}$ jets to protostars is roughly one to one, though the number of protostars is again low.

However, neither of the above semi-quantitative observations seems to be related to clustering. We demonstrate this by measuring the radius to the 5 th, 6 th, 7 th, 10 th and 20 th nearest neighbour (NN) for each of the protostars identified in Orion A. The distribution of 6th NN radii is displayed in Fig. 19, where we distinguish protostars that do drive $\mathrm{H}_{2}$ flows from those that do not. We also separate protostars north of Dec $-6^{\circ}$ (the OMC 2/3 region) from those south of Dec $-6^{\circ}$ (L 1641). In Table 1 we list the mean and median radius for each sample of Nth nearest neighbour radii, and again distinguish outflow sources from the remaining sample of protostars, and contrast the northern region with the south (median values tend to exclude outliers with large radii located on the periphery of each region). Table 1 and Fig. 19 both demonstrate that there is no significant difference between the spatial distribution of protostars that drive $\mathrm{H}_{2}$ flows and protostars that do not. In other words, the molecular outflow sources are no more - nor no less - clustered than the sources that don't drive $\mathrm{H}_{2}$ flows. This result is confirmed by $\mathrm{K}-\mathrm{S}$ testing: for the 6 th $\mathrm{NN}$ radii, in the northern region there is a $71 \%$ probability that the protostar and $\mathrm{H}_{2}$-outflow-driving source radii are drawn from the same sample; in the south the probability is lower (11\%) though still inconclusive - both distributions of NN radii are essentially the same. K-S tests for the 5 th and 7 th $\mathrm{NN}$ radii yield similar results.

We conclude that the differences we note at the beginning of this section are simply due to evolutionary effects. There are clearly regions within Orion A that are more evolved than others; young regions like L 1641-N that contain embedded protostars that drive $\mathrm{H}_{2}$ outflows, and older regions like L 1641-C with clusters of "protostars" that are not associated with large clouds, dense cores or molecular outflows. The high fraction of $\mathrm{H}_{2}$-outflow-driving protostars suggests that transition time scales - from protostar with $\mathrm{H}_{2}$ flow, via protostar without $\mathrm{H}_{2}$ flow, to disc-excess source - are relatively brief. The fact that the protostars without molecular outflows are no more widely distributed (on average) than protostars with outflows suggests that the protostellar phase as a whole is short in comparison to the overall time-scale over which young stars become more widely distributed.

Finally, the NN analysis summarised in Table 1 also demonstrates that there are considerable differences between OMC 2/3 and $\mathrm{L}$ 1641. All protostars, regardless of whether they drive $\mathrm{H}_{2}$ outflows or not, are far more clustered in the north (NN radii are consistently shorter) than in L 1641 in the south. Clustering, the distributions of protostars and disc-excess sources, and the population of young stars throughout Orion, will be discussed in detail in a forthcoming paper (Megeath et al., in prep.).

\section{Summary and conclusions}

We present near-IR images of $\mathrm{H}_{2}$ outflows spread across an eight square degree region that encompasses most of the Orion A GMC. For much of this region Spitzer photometry is used to identify protostars and disc-excess sources, and large-scale (sub)millimetre maps are used to plot the distribution of molecular gas. Tangential velocities (proper motions) are measured for features in 33 outflows; these data are used to help associate the outflows with the protostars and dense molecular cores.

We identify 43 new $\mathrm{H}_{2}$ outflows, increasing the number of $\mathrm{H}_{2}$ flows in Orion A to 116 (not including the Orion bullets). From the Spitzer sample of young stars we find sources for most of these outflows. Of the 300 or so protostars identified by Megeath et al. (in prep.), at least $1 / 3$ seem to be driving $\mathrm{H}_{2}$ flows. Indeed, the $\mathrm{H}_{2}$ outflow sources are predominantly protostars (Class 0/I sources) rather than disc-excess sources (mostly Class II T Tauri stars), though a few of the latter source sample do seem to power $\mathrm{H}_{2}$ flows. Most $\mathrm{H}_{2}$ outflow sources have positive spectral indices and are associated with dust cores; most molecular cores that harbour protostars also drive molecular outflows. All evidence points to the extreme youth of $\mathrm{H}_{2}$ jet progenitors.

We find no evidence for a preferred molecular outflow direction. Even in OMC 2/3, the region north of the ONC, where the molecular gas is confined to a north-south chain of dense cores, the $\mathrm{H}_{2}$ flows seem to be randomly orientated. Nor do we find a correlation between $\mathrm{H}_{2}$ flow length or opening angle and source spectral index or core flux at $1200 \mu \mathrm{m}$. We find no evidence that "more evolved" sources, i.e. those with low $\alpha$ or weak $1200 \mu \mathrm{m}$ emission, drive longer or less-well-collimated $\mathrm{H}_{2}$ flows. The caveats associated with measuring flow parameters from $\mathrm{H}_{2}$ observations are discussed in light of these results.

Finally, we investigate the spatial distribution of $\mathrm{H}_{2}$ outflow sources in relation to the overall distribution of protostars. Although well-known star clusters, such as L 1641-N and L 1641-C, are associated with clusters of a dozen or so protostars and disc-excess sources, only groups or clusters of protostars that are still surrounded by dense molecular gas seem to be associated with multiple $\mathrm{H}_{2}$ flows. Protostars that power $\mathrm{H}_{2}$ outflows 


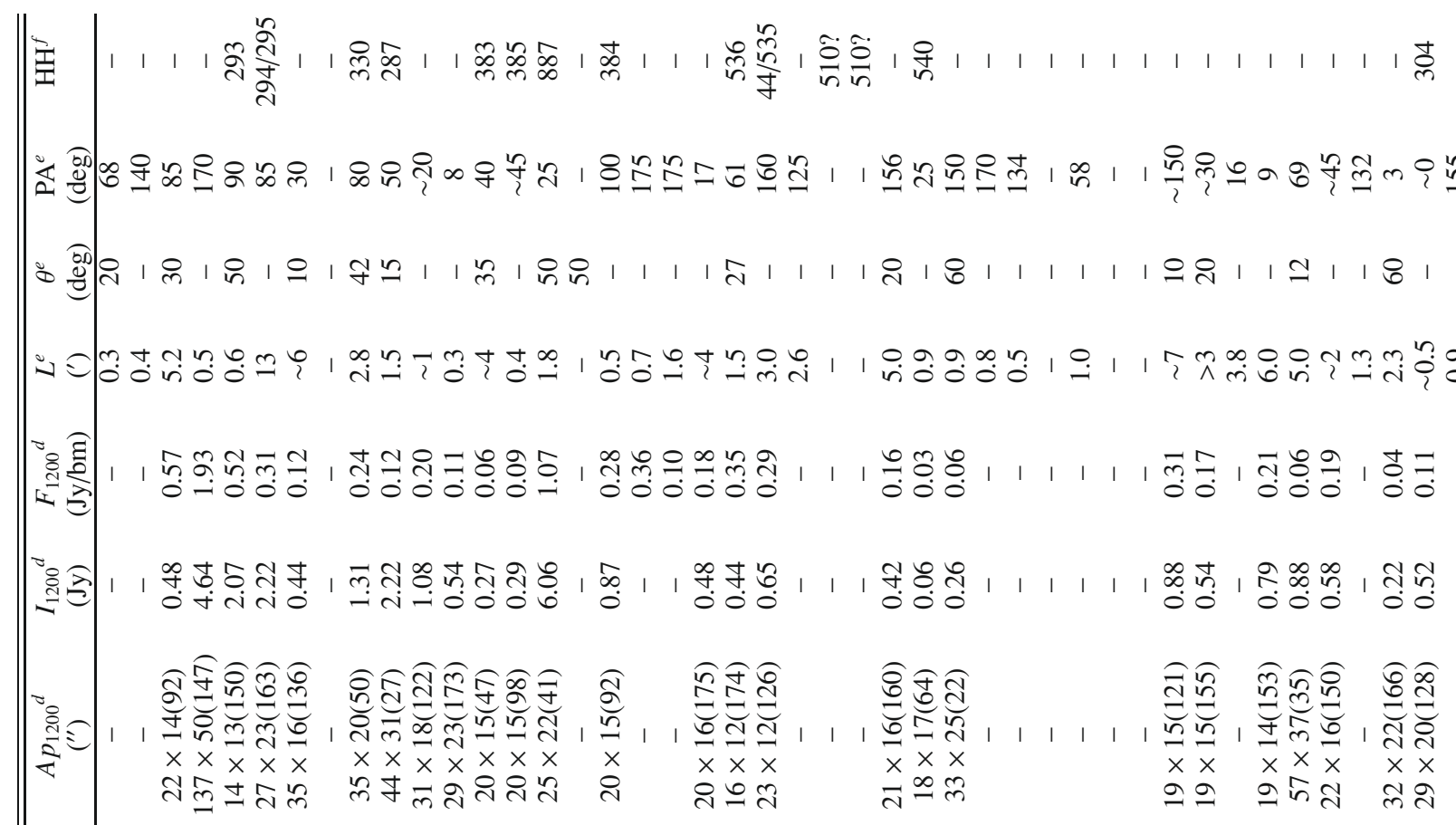

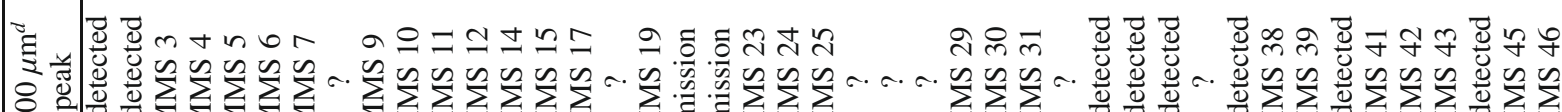

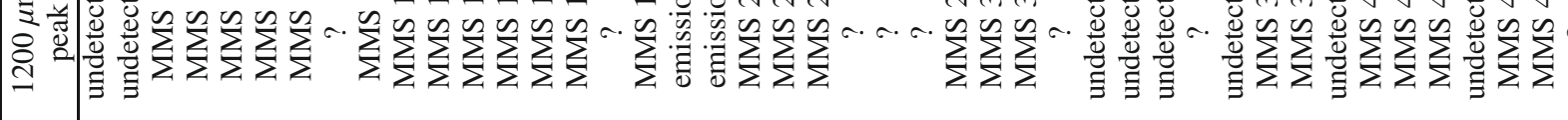

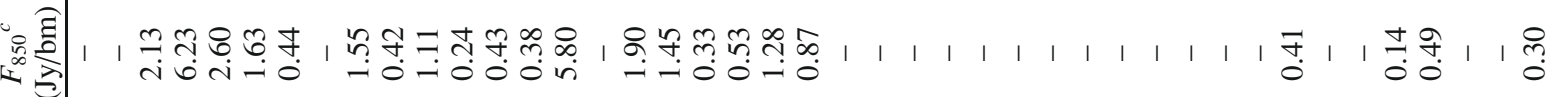

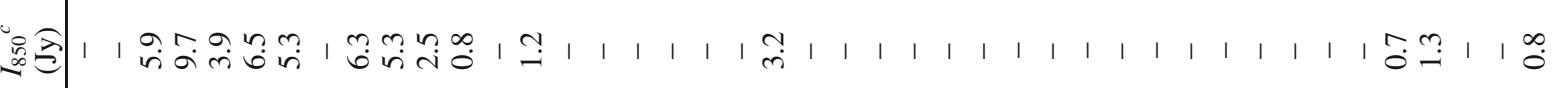

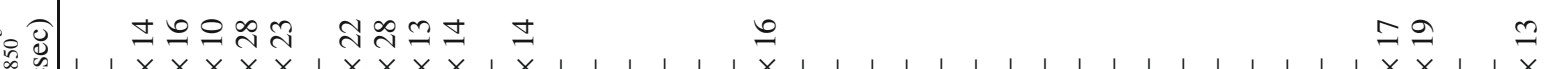

过這

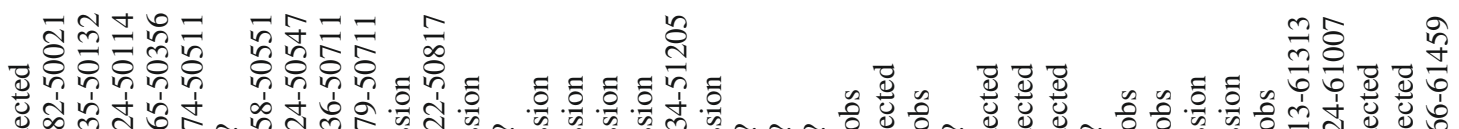

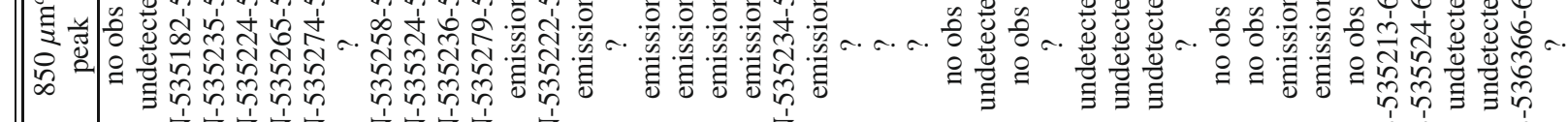

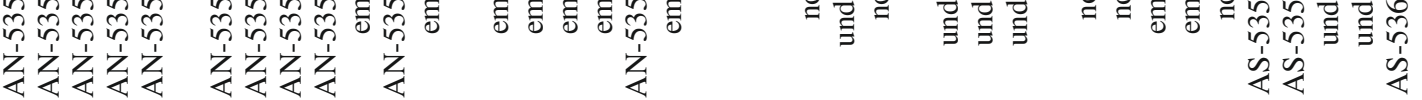

\& 金苛

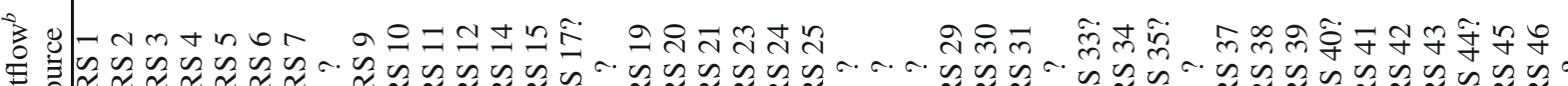

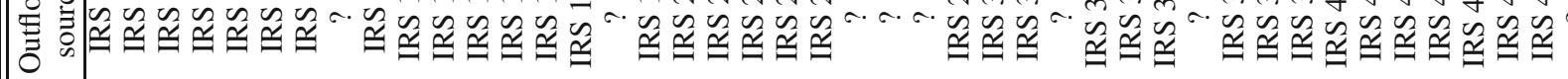

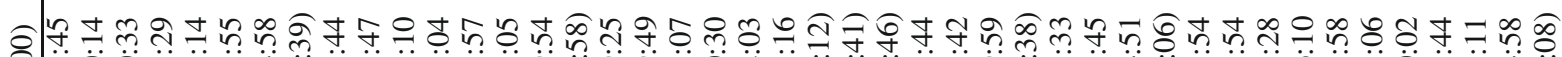

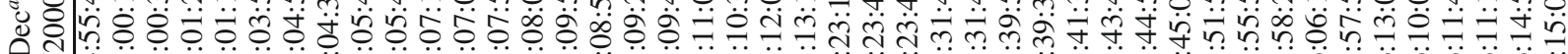

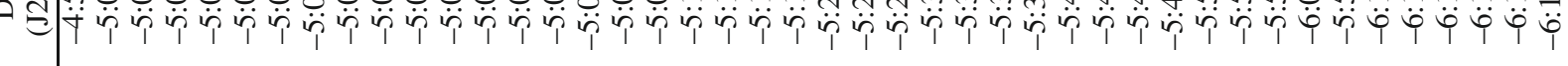

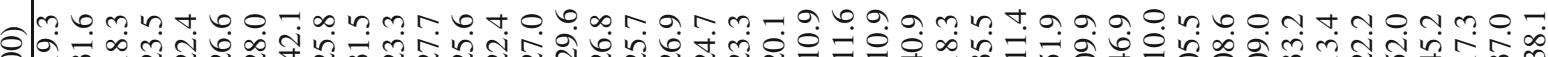
< \& |새 


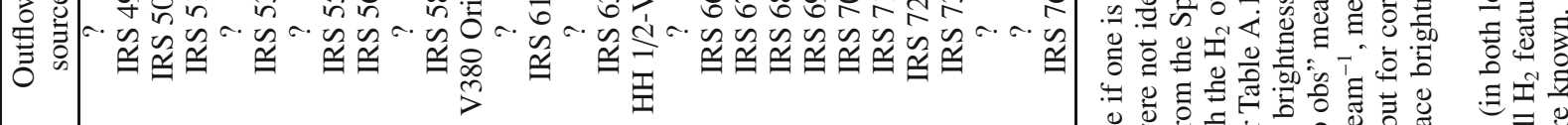

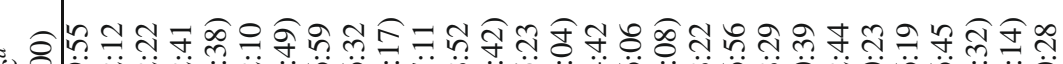
ڤั 
are no more (nor no less) clustered than protostars that do not. This, together with the fact that a high percentage of protostars do drive $\mathrm{H}_{2}$ flows, suggests that the $\mathrm{H}_{2}$-outflow-driving phase of a protostar's early evolution is only marginally shorter than the overall lifetime of the protostar; it seems clear that $\mathrm{H}_{2}$ jets fade very quickly as each source evolves towards the Class II pre-main-sequence phase.

Acknowledgements. We gratefully acknowledge the two referees, Bo Reipurth and John Bally, for their careful reading of the text and very useful comments, which served to improve the accuracy, clarity and overall quality of the paper. Thanks are also due to Dave Nutter for making the SCUBA data available to us. We have made extensive use of the Starlink analysis tools GAIA and TOPCAT (http: //starlink. jach.hawaii.edu/). We acknowledge the Cambridge Astronomical Survey Unit (CASU) for processing the near-IR data, and the WFCAM Science Archive in Edinburgh for making the bulk of the WFCAM data discussed here available to us. The United Kingdom Infrared Telescope is operated by the Joint Astronomy Centre on behalf of the UK Particle Physics and Astronomy Research Council. Some of the WFCAM data reported here were obtained as part of the UKIRT Service Programme. This research made use of data products from the Spitzer Space Telescope Archive. These data products are provided by the services of the Infrared Science Archive operated by the Infrared Processing and Analysis Centre/California Institute of Technology, funded by the National Aeronautics and Space Administration and the National Science Foundation. We have also made much use of the SIMBAD database, operated at CDS, Strasbourg, France.

\section{References}

Allen, L. E., \& Davis, C. J. 2008, in Handbook of Star Forming Regions Vol I, ed. B. Reipurth, ASP Monographs, 621

Allen, L. E., Calvet, N., D’Alessio, P., et al. 2004, ApJS, 154, 363

Anathpindika, S., \& Whitworth, A. P. 2008, A\&A, 487, 605

Anglada, G., Villuendas, E., Estalella, R., et al. 1998, AJ, 116, 2953

Antoniucci, S., Nisini, B., Giannini, T., \& Lorenzetti, D. 2008, A\&A, 479, 503

Arce, H. A., \& Sargent, A. I. 2006, ApJ, 646, 1070

Arce, H. A., Shepherd, D., Gueth, F., et al. 2007, in Protostars and Planets V, ed. B. Reipurth, D. Jewitt, \& K. Keil (Tucson: University of Arizona Press), 245

Aspin, C. A., \& Reipurth, B. 2000, MNRAS, 311, 522

Bally, J., \& Devine, D. 2001, ApJ, 546, 299

Bally, J., O’Dell, C. R., \& McCaughrean, M. J. 2000, AJ, 119, 2919

Bally, J., Heathcote, S., Reipurth, B., et al. 2002, AJ, 123, 2627

Banerjee, R., \& Pudritz, R. E. 2006, ApJ, 641, 949

Beck, T. L. 2007, AJ, 133, 1689

Caratti O Garatti, A., Froebrich, D., Eislöffel, J., Giannini, T., \& Nisini, B. 2008 , A\&A, 485, 137

Carpenter, J. M., Hillenbrand, L. A., \& Skrutskie, M. F. 2001, AJ, 121, 3160

Casali, M., Adamson, A., Alves de Oliveira, C., et al. 2007, A\&A, 467, 777

Cavanagh, B., Hirst, P., Jenness, T., et al. 2003, ASPC, 295, 237

Chini, R., Reipurth, B., Ward-Thompson, D., et al. 1997, ApJ, 474, L135

Crutcher, R. M., Troland, T. H., Lazareff, B., Paubert, G., \& Kazés, I. 1999, ApJ, 514, L121

Davis, C. J., Eislöffel, J., \& Ray, T. P. 1994, ApJ, 426, L93

Davis, C. J., Dent, W. R. F., Matthews, H. E., Coulson, I. M., \& McCaughrean, M. J. 2000, MNRAS, 318, 952

Davis, C. J., Kumar, M. S. N., Sandell, G., et al. 2007, MNRAS, 374, 29

Davis, C. J., Scholz, P., Lucas, P. W., \& Adamson, A. 2008, MNRAS, 387, 954

Devine, D., Bally, J., Reipurth, B., \& Heathcote, S. 1997, AJ, 114, 2095

Doppmann, G. W., Jaffe, D. T., \& White, R. J. 2003, AJ, 126, 3042

Doppmann, G. W., Greene, T. P., Covey, K. R., \& Lada, C. J. 2005, AJ, 130, 1145

Dye, S., Warren, S. J., Hambly, N. C., et al. 2006, MNRAS, 372, 1227

Eislöffel, J., \& Mundt, R. 1997, AJ, 114, 280

Eislöffel, J., Mundt, R., \& Böhm, K.-H. 1994, AJ, 108, 1042
Enoch, M. L., Evans, N. J., Sargent, A. I., et al. 2008, ApJ, 684, 1240 Fazio, G. G., Hora, J. L., Allen, L. E., et al. 2004, ApJS, 154, 10 Froebrich, D. F., Schmeja, S., Smith, M. D., \& Klessen, R. S. 2006, MNRAS, 368,435

Gålfalk, M., \& Olofsson, G. 2007, A\&A, 466, 579

Greene, T. P., \& Lada, C. J. 2002, AJ, 124, 2185

Gutermuth, R. A., Myers, P. C., Megeath, S. T., et al. 2008, ApJ, 674, 336

Gutermuth, R. A., Megeath, S. T., Myers, P. C., et al. 2009, ApJS, submitted

Hatchell, J., Fuller, G. A., Richer, J. S., Harries, T. J., \& Ladd, E. F. 2007, A\&A, 468,1009

Hester, J. J., Stapelfeldt, K. R., \& Scowen, P. A. 1998, AJ, 116, 372

Hewett, P. C., Warren, S. J., Leggett, S. K., \& Hodgkin, S. T. 2006, MNRAS, 367,454

Heyer, M. H., Strom, S. E., \& Strom, K. M. 1987, AJ, 94, 1653

Houde, M., Dowell, C. D., Hildebrand, R. H., et al. 2004, ApJ, 604, 717

Kreysa, E., Gemünd, H.-P., Gromke, J., et al. 1999, Infrared Physics and Technology, 40, 191

Johnstone, D., \& Bally, J. 1999, ApJ, 510, L49

Johnstone, D., \& Bally, J. 2006, ApJ, 653, 383

Jørgensen, J. K., Johnstone, D., Kirk, H., \& Myers, P. C. 2007, ApJ, 656, 293

Kassis, M., Adams, J. D., Campbell, M. F., et al. 2006, ApJ, 637, 823

Kumar, M. S. N, Davis, C. J., Grave, J. M. C., Ferreira, B., \& Froebrich, D. 2007, MNRAS, 374, 54

Kutner, M. L., Tucker, K. D., Chin, G., \& Thaddeus, P. 1977, ApJ, 215, 521

Larry, K. 2008, ApJ, in press

Lee, J.-K., \& Burton, M. G. 2000, MNRAS, 315, 11

Lee, C.-F., Mundy, L. G., Stone, J. M., \& Ostriker, E. C. 2002, ApJ, 576, 294

Matthews, B. C., Fiege, J. D., \& Moriarty-Schieven, G. 2002, ApJ, 569, 304

Matthews, B. C., Wilson, C. D., \& Fiege, J. D. 2001, ApJ, 562, 400

Megeath, S. T., Allen, L. E., Gutermuth, R. A., et al. 2004, ApJS, 154, 367

Megeath, S. T., Allgaier, E., Young, E., et al. 2009, AJ, submitted

Ménard, F., \& Duchéne, G. 2004, Ap\&SS, 292, 419

Mouschovias, T. 1976, ApJ, 207, 141

Nutter, D., \& Ward-Thompson, D. 2007, MNRAS, 374, 1413

Peterson, D., \& Megeath, S. T. 2008, in Handbook of Star Forming Regions Vol I, ed. B. Reipurth, ASP Monographs, 590

Reipurth, B. 1985, A\&AS, 61, 319

Reipurth, B. 1989, A\&A, 220, 249

Reipurth, B., \& Bally, J. 2001, ARA\&A, 39, 403

Reipurth, B., Bally, J., \& Devine, D. 1997, AJ, 114, 2708

Reipurth, B., Devine, D., \& Bally, J. 1998, AJ, 116, 1396

Reipurth, B., Heathcote, S., Yu, K. C., Bally, J., \& Rodríguez, L. F. 2000, ApJ, 534,317

Rieke, G. H., Young, E. T., Engelbracht, C. W., et al. 2004, ApJSS, 154, 25

Sakamoto, S., Hayashi, M., Hasegawa, T., Handa, T., \& Oka, T. 1994, ApJ, 425, 641

Schild, H., Miller, S., \& Tennyson, J. 1997, A\&A, 318, 608

Simpson, J. P., Colgan, S. W. J., Erickson, E. F., Burton, M. G., \& Schultz, A. S. B. 2006, ApJ, 642, 339

Slesnick, C. L., Hillenbrand, L. A., \& Carpenter, J. M. 2004, ApJ, 610, 1045

Smith, M. D., \& Rosen, A. 2005, MNRAS, 357, 1370

Stanke, T. 2000, Ph.D. Thesis, Universität Potsdam

Stanke, T., \& Williams, J. P. 2007, AJ, 133, 1320

Stanke, T., McCaughrean, M. J., \& Zinnecker, H. 2002, A\&A, 392, 239 (Sta02)

Strom, K. M., Strom, S. E., Wolff, S. C., Morgan, J., \& Wenz, M. 1986, ApJS, 62,39

Tamura, M., Kandori, R., Kusakabe, N., et al. 2006, ApJ, 649, L29

Usuda, T., Sugai, H., Kawabata, H., et al. 1996, ApJ, 464, 818

Yu, K. C., Bally, J., \& Devine, D. 1997, ApJ, 485, L45

Yu, K. C., Billawala, Y., Smith, M. D., Bally, J., \& Butner, H. M. 2000, AJ, 120, 1974

Vallée, J. P., \& Fiege, J. D. 2006, ApJ, 636, 332

Velusamy, T., Langer, W. D., \& Marsh, K. A. 2007, ApJ, 668, L159

Whitney, B. A., Wood, K., Bjorkman, J. E., \& Cohen, M. 2003, ApJ, 598, 1079

Whitney, B. A., Indebetouw, R., Bjorkman, J. E., \& Wood, K. 2004, ApJ, 617, 1177

Williams, J. P., Plambeck, R. L., \& Heyer, M. H. 2003, ApJ, 591, 1025 


\section{Appendix A: Description of the $\mathrm{H}_{2}$ outflows in Orion A}

Here we give a brief description of the $\mathrm{H}_{2}$ flows tabulated in Tables 2 and 3, relating each jet to its probable driving source and associated dense core (as listed in Tables 2 and 3). We focus on the newly-discovered "DFS" $\mathrm{H}_{2}$ flows, though we also discuss "SMZ" flows were our PM measurements and/or comparison with $1200 \mu \mathrm{m}$ data help identify the driving source (see Sta02 for additional information on the SMZ jets).

In this section we also present continuum-subtracted $\mathrm{H}_{2} 2.122 \mu \mathrm{m}$ images for the DFS $\mathrm{H}_{2}$ flows (most of the SMZ jets are labelled in the figures in the main text; or see Sta02). Note that the micro-stepped WFCAM images have in these Appendix figures only been binned over $2 \times 2$ pixels, to a plate scale of $0.4^{\prime \prime}$, to improve signal-to-noise but also to retain spatial resolution. In each figure we mark the positions of Spitzer-identified protostars and label prominent $\mathrm{HH}$ objects.

\section{A.1. OMC 2/3 and NGC $1977\left(-4.3^{\circ}>\delta>-5.4^{\circ}\right)$}

\section{A.1.1. SMZ 1 to SMZ 5 and DFS 101}

At the northern end of Orion $\mathrm{A}$, in addition to the five $\mathrm{H}_{2}$ flows identified by Sta02 (SMZ 1 to SMZ 5), we note the new, curving $\mathrm{H}_{2}$ flow, DFS 101, situated $\sim 15^{\prime}$ to the northwest of Haro $5 \mathrm{a} / 6 \mathrm{a}$ (Fig. A.1a). Although we have no (sub)millimetre observations in this region, the outflow source is clearly identified by Spitzer as a protostar. Indeed, Spitzer IRS sources are identified for all six $\mathrm{H}_{2}$ flows; the sources of jets SMZ 3, 4 and 5 are also associated with a chain of dust cores. SMZ 3 is particularly impressive and may in fact comprise multiple flows, driven by two or more of the Spitzer protostars in this region.

\section{A.1.2. SMZ 6 to SMZ 10 and DFS 102}

The prominent $\mathrm{H}_{2}$ jets labelled SMZ 6 and SMZ 9 by Sta02 are centred on MAMBO/SCUBA cores and a number of Spitzer protostars (see Figs. 2 and 3 in the main body of the paper). SMZ 6 is associated with the bipolar nebula and candidate Fu Ori source Haro $5 \mathrm{a} / 6 \mathrm{a}$. The western lobe of the flow is delineated by a faint $\mathrm{H}_{2}$ bow situated $\sim 3^{\prime}$ to the west; the parsec-scale jet may also be associated with $\mathrm{HH}$ 41, HH 42, HH 128 and HH 129 (Reipurth et al. 1997). The reddened source of this outflow is partially visible to the east of the band of obscuration that separates the two lobes of the nebula.

IRS 10 is enveloped in a conical nebula that opens out toward the west. Although this source is well aligned with the SMZ 9 flow axis, high-resolution CO maps reveal a compact bipolar CO outflow centred on IRS 10 that is instead aligned with SMZ 10 (Yu et al. 2000; Williams et al. 2003). IRS 9 is perhaps the best candidate driving source for SMZ 9 (which is also associated with a high-velocity $\mathrm{CO}$ lobe). The PMs of the $\mathrm{H}_{2}$ knots in SMZ 9, which are generally moving westward with a velocity of 100-150 $\mathrm{km} \mathrm{s}^{-1}$, certainly support this interpretation.

$\mathrm{HH} 42$ lies about $5^{\prime}$ east of Haro 5a/6a. Bright $\mathrm{H}_{2}$ emission is detected from this object. We also find a candidate outflow source along the flow axis (IRS 102), and therefore identify these features with a new $\mathrm{H}_{2}$ jet, DFS 102 (Fig. A.1c).

The PM of the nebulous, bow-shaped $\mathrm{H}_{2}$ feature SMZ 8 is unfortunately inconclusive, and this flow has no obvious progenitor. The faint though well-collimated SMZ 7 jet is associated with a faint conical nebula and Spitzer protostar (IRS 7).

\section{A.1.3. SMZ 11 to SMZ 20 and DFS 103}

Based on PMs, the source of SMZ 11 (and SMZ 13) probably lies mid-way along this chain of $\mathrm{H}_{2}$ knots. A reddened protostar (IRS 11) is visible in the colour images in Fig. 2 which is also associated with a molecular core.

SMZ 14 and SMZ 16 may be the lobe and counter-lobe of another large flow. The PM of the outer conical bow in SMZ 14 is toward the north-northeast, as expected.

IRS 17 is a possible candidate for the spectacular $\mathrm{H}_{2}$ jet SMZ 17 - it coincides with the peak in the $1200 \mu \mathrm{m}$ map and a dark lane in our colour image in Fig. 2 - although there are a number of candidate protostars in this nebulous region. SMZ 18 may be part of the same outflow, or may indeed be an unrelated finger or "bullet".

SMZ 12, SMZ 15, SMZ 19 and SMZ 20 are compact features or small chains of $\mathrm{H}_{2}$ knots. Sources are identified for each outflow.

DFS 103 is a pair of faint knots close to a Spitzer disc source (Fig. A.1d).

\section{A.1.4. SMZ 21 to SMZ 28, DFS 104 and DFS 105}

Moving southward through the OMC 2/3 region, as we approach the brighter regions in the Orion nebula, the identification of outflow source candidates becomes more difficult. Even so, reddened sources are identified for all of the $\mathrm{H}_{2}$ flows.

The chain of knots and bow-shocks that comprise SMZ 23 are likely driven by one of the protostars to the north, possibly the Spitzer source/dust core IRS 23. The leading bow in SMZ 23 does appear to be moving southward with a PM of $\sim 100 \mathrm{~km} \mathrm{~s}^{-1}$, consistent with its morphology and this interpretation.

Features SMZ 21 and SMZ 22 may be part of yet another $\mathrm{H}_{2}$ flow, driven by the disc-excess source IRS 21. Both have PMs directed southward.

SMZ 24 has well-defined PMs directed away from the protostar IRS 24; the tangential velocities of the features in SMZ 25 are imprecise, though IRS 25 remains a good source candidate.

SMZ 26-28 are $\mathrm{H}_{2}$ features identified by $\mathrm{Sta} 02$ that lie along the western edge of the Orion Nebula and may in some cases be "Orion Bullets"; we are unable to identify candidate outflow sources or dust cores because of the bright emission associated with this region.

DFS 104 are faint $\mathrm{H}_{2}$ objects near a Spitzer disc source; DFS 105 is a faint arc located southwest of the ONC. Both have candidate driving sources and are shown in Fig. A.1.

\section{A.2. The $\mathrm{OMC} 4 / 5$ region $\left(-5.4^{\circ}>\delta>-6.3^{\circ}\right)$}

\section{A.2.1. SMZ 29 to SMZ 36 and DFS 106 to DFS 112}

The $\mathrm{H}_{2}$ flow features just to the south of the ONC are generally faint and compact. The newly-identified outflows are shown in Figs. A.2-A.3.

DFS 106, DFS 107 and DFS 108 all have candidate driving source; DFS 109 comprises two well-defined bow shocks east of the chain of dense cores; DFS 110 is a cluster of $\mathrm{H}_{2}$ knots to the west of the main L 1641 ridge. DFS 110 is beyond the bounds of the MAMBO and SCUBA observations, though candidate sources are evident in the Spitzer data; this cluster of knots is also associated with the T Tauri star Haro 4-145 and a molecular outflow (Fig. A.2d).

DFS 111 and DFS 112 are compact, bow-shaped $\mathrm{H}_{2}$ features with candidate sources in the Spitzer sample (Figs. A.3a and b). 

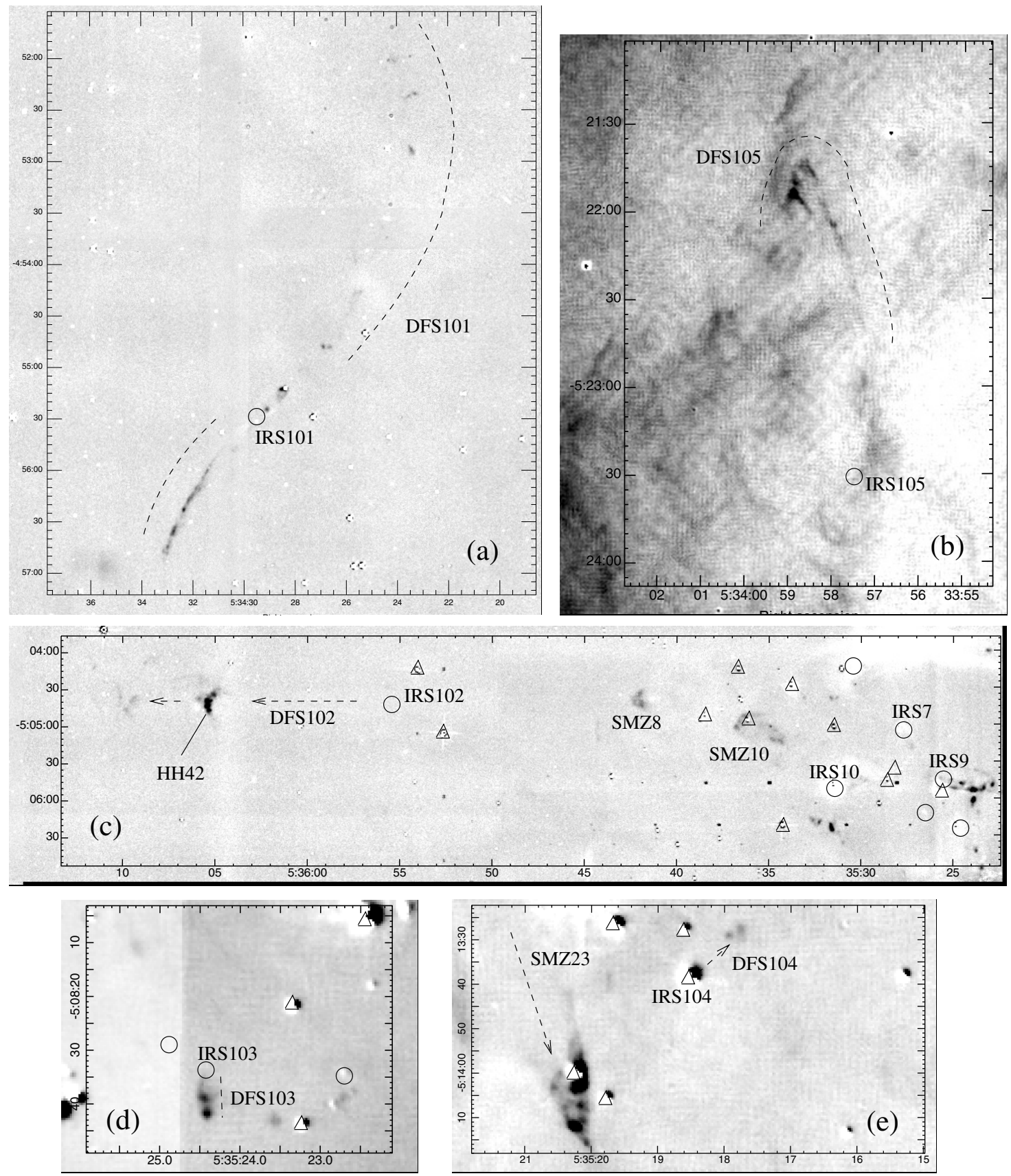

Fig. A.1. Continuum-subtracted $\mathrm{H}_{2} 2.122 \mu \mathrm{m}$ images of DFS 101-105. Spitzer-identified young stars are marked with circles (protostars) and triangles (disc-excess sources).

We have attempted to measure PMs for the knots in DFS 107, DFS 108, DFS 109 and DFS 111, as well as SMZ 31 to SMZ 36 (SMZ 29 and SMZ 30 are faint, diffuse features, though SMZ 29 is clearly driven by a nebulous $K$-band source, dust core and Spitzer protostar). The PM results are in many cases marginal since most of the features are rather faint and/or diffuse. Variability will also affect our PM measurements. This probably explains why only DFS 109, SMZ 31 and SMZ 35 have PMs that are consistent with them being driven by their identified progenitors (in this case IRS 109, IRS 31 and IRS 35, respectively).

\section{A.2.2. SMZ 37 to SMZ 44, DFS 113 and DFS 114}

Moving south through the NGC 1980 region toward OMC 5, there are a number of $\mathrm{H}_{2}$ knots around the three Spitzer pro- tostars/molecular cores, IRS 38, IRS 39 and IRS 41 (Fig. 5). It seems likely that all three embedded sources have $\mathrm{H}_{2}$ flows. IRS 41 probably drives the distant bow shock SMZ 41 to the south; similarly, IRS 40 may drive the bow shock SMZ 40.

PMs for the $\mathrm{H}_{2}$ features in the orthogonal flows SMZ 38 and SMZ 39 confirm the proposed associations with their Spitzer protostars; the PMs of SMZ 37, SMZ 40 and SMZ 41 are unfortunately inconclusive or below the errors.

SMZ 42 represents a classic example of low-mass star formation (Fig. 5). It consists of a collimated, knotty $\mathrm{H}_{2}$ jet terminating in a sweeping $\mathrm{H}_{2}$ bow shock. A fainter bow equidistant from the source marks the end of the counter-lobe. The Spitzeridentified outflow source, IRS 42, is also coincident with a small dust core. 

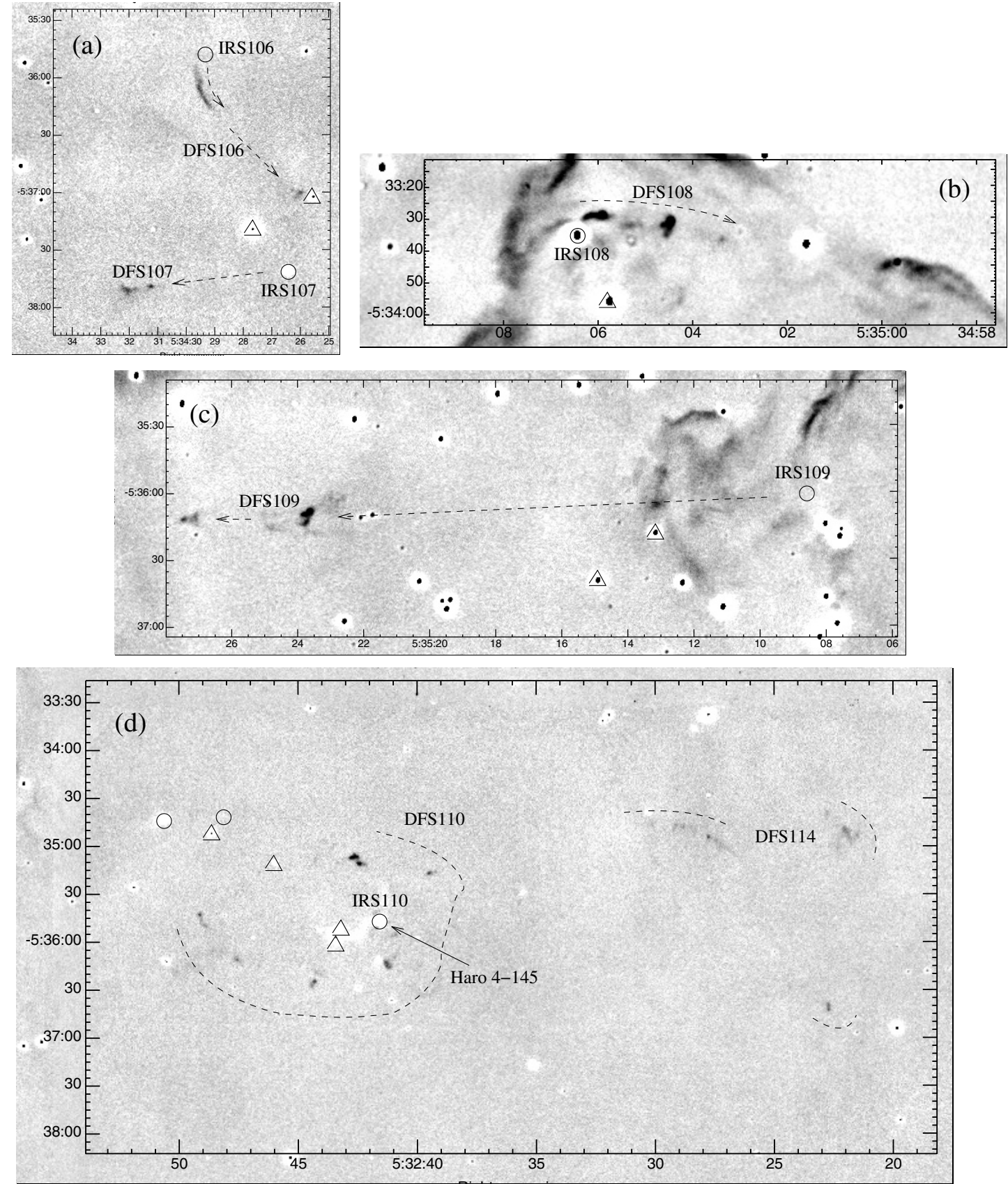

Fig. A.2. Continuum-subtracted $\mathrm{H}_{2} 2.122 \mu$ m images of DFS 106-110 and DFS 114. Protostars and disc-excess sources are marked. DFS 108 coincides with the irradiated jet features HH 541 and HH 882.

SMZ 43 is associated with the variable flare star V1296 Ori and a compact peak at $1200 \mu \mathrm{m}$; SMZ 43 may be a PhotoDissociation Region (PDR) rather than an outflow. SMZ 44 is a faint $\mathrm{H}_{2}$ bow and cavity that we tentatively associated with the Spitzer disc source IRS 44.

DFS 113 is a very faint bow-shaped feature $\sim 3.5^{\prime}$ south of IRS 113 (Fig. A.3c), while DFS 114 is a group of knots and filaments a few arcminutes west of DFS 110 and Haro 4-145 (labelled in Fig. A.2d).

\section{A.3. $L$ 1641-N and NGC $1999\left(-6.3^{\circ}>\delta>-7.0^{\circ}\right)$}

L 1641-N and NGC 1999 are associated with many well-known HH objects (HH 1/2, HH33/40, HH 34, etc.), most of which are labelled in Figs. 6-8 in the main text.

\section{A.3.1. SMZ 45 to SMZ 54, SMZ 57, SMZ 58, DFS 115 and DFS 118}

Candidate $\mathrm{H}_{2}$ outflow sources are identified for SMZ 45 and SMZ 46, though not for the chain of diffuse knots in SMZ 47 (see Sta02 for details) or for the elongated knots in SMZ 48 (HH 299).

The parsec-scale $\mathrm{H}_{2}$ flow SMZ 49 is driven by a source in the L 1641-N cluster. There are five Spitzer protostars in L 1641-N (Fig. A.4b). IRS 49 is best aligned with SMZ 49 and with the PM vectors of features along this axis (Gålfalk \& Olofsson 2007). However, IRS 49 is offset by $\sim 10^{\prime \prime}$ to the east-southeast of the brightest peak in $1200 \mu \mathrm{m}$ emission, which coincides with a second source, IRS 53. This embedded protostar probably drives the $\mathrm{H}_{2} / \mathrm{CO}$ outflow SMZ 53 (see also Stanke \& Williams 2007). 

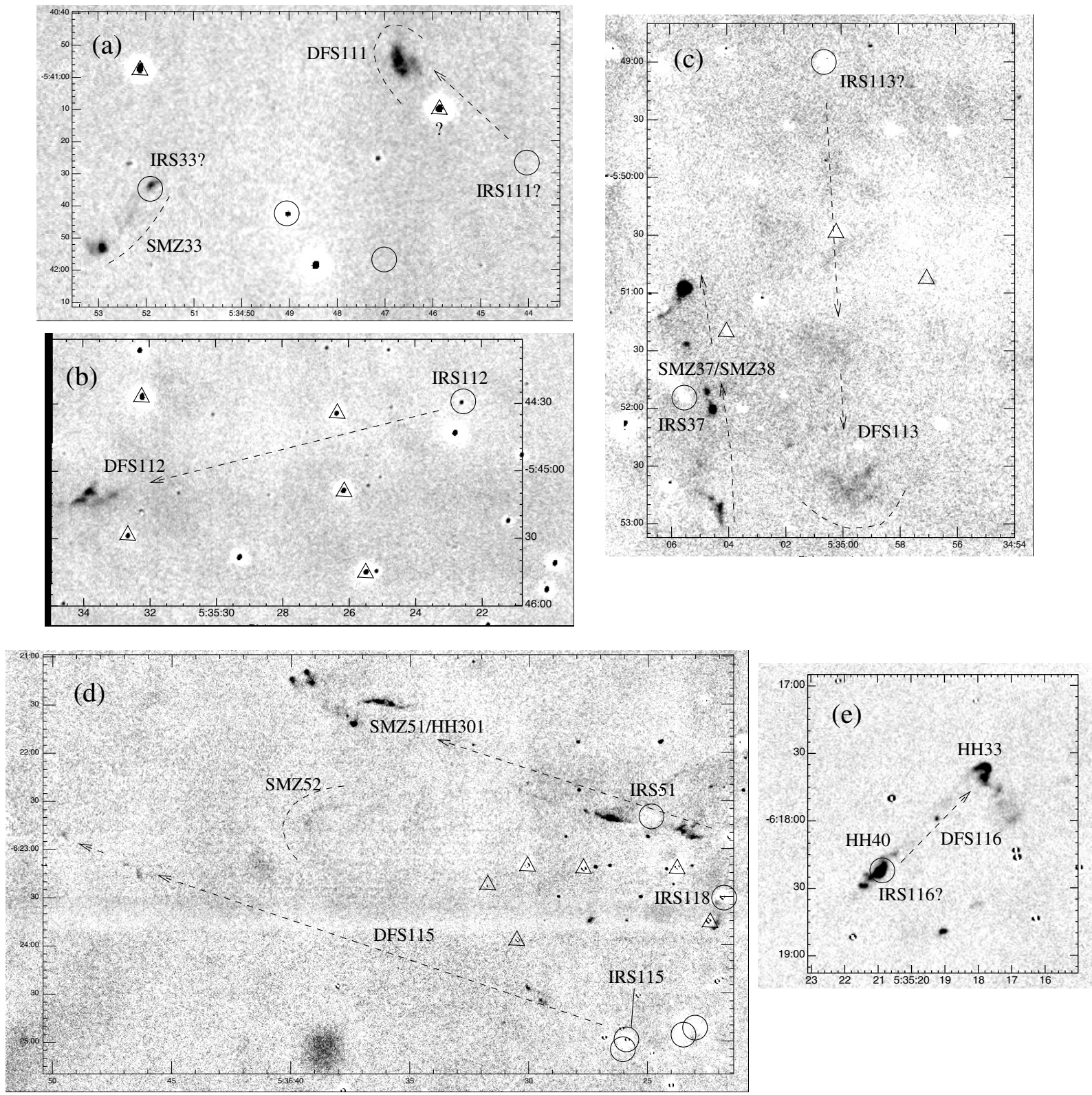

Fig. A.3. Continuum-subtracted $\mathrm{H}_{2} 2.122 \mu$ m images of DFS 111-113, DFS 115 and DFS 116 (better known as HH 33/40). Protostars and discexcess sources are marked.

The other sources in the cluster may also power some of the $\mathrm{H}_{2}$ features in $\mathrm{L} 1641-\mathrm{N}$.

SMZ 50 is possibly driven by the disc source IRS 50 and, like Gålfalk \& Olofsson (2007), we associate SMZ 51 (HH 301) with another Spitzer source, the protostar IRS 51 (Figs. A.3d and A.4b). SMZ 52 is a faint arc, possibly part of the DFS 115 flow.

SMZ 58 is a nice example of a bright, bipolar $\mathrm{H}_{2}$ flow associated with a Spitzer protostar and a compact, dense core. SMZ 57 is a series of $\mathrm{H}_{2}$ knots with no obvious source; it is located $\sim 1.5^{\prime}$ north of SMZ 58.

Finally, we identify two other $\mathrm{H}_{2}$ flows in L $1641 \mathrm{~N}$, DFS 115 and DFS 118. DFS 118 comprises compact arcuate features that are associated with a bipolar CO outflow (Stanke \& Williams 2007); this flow is driven by IRS 118 (Fig. A.4b). A small cluster of protostars $\sim 3^{\prime}$ south of L 1641-N, known as Strom 11, includes the source of the other outflow, DFS 115 (Fig. A.3d).

\section{A.3.2. HH 34 (SMZ 55 and SMZ 56), HH 33/40 (DFS 116) and $\mathrm{HH} 83 / 84$ (DFS 117)}

To the west of L 1641-N, three Spitzer protostars are identified in the vicinity of the archetypal jet HH 34. HH 34-IRS itself (IRS 55) coincides with the local peak in $1200 \mu \mathrm{m}$ dust emission; a second source, IRS 56, also appears to drive a collimated molecular jet.

Roughly $10^{\prime}$ to the north, $\mathrm{HH} 40$ is also identified as a Spitzer protostar, which we label IRS 116. As noted in the main text, this source could be powering HH 33 and HH 40 (Fig. A.3e).

Further west, DFS 117 comprises a collimated optical jet ( $\mathrm{HH} 83$ ) and a group of arcuate $\mathrm{H}_{2}$ knots $10.5^{\prime}$ to the south-east (HH 84). We note that HH 84 could be driven by a neighbouring protostar or disc source (Fig. A.4a). HH 83-IRS is outside the bounds of the SCUBA, MAMBO and two of the four Spitzer IRAC bands; in the remaining two bands, this object has a colour consistent with it being a disc-excess source. 
C. J. Davis et al.: A census of molecular hydrogen outflows and their sources along the Orion A molecular ridge, Online Material p 5
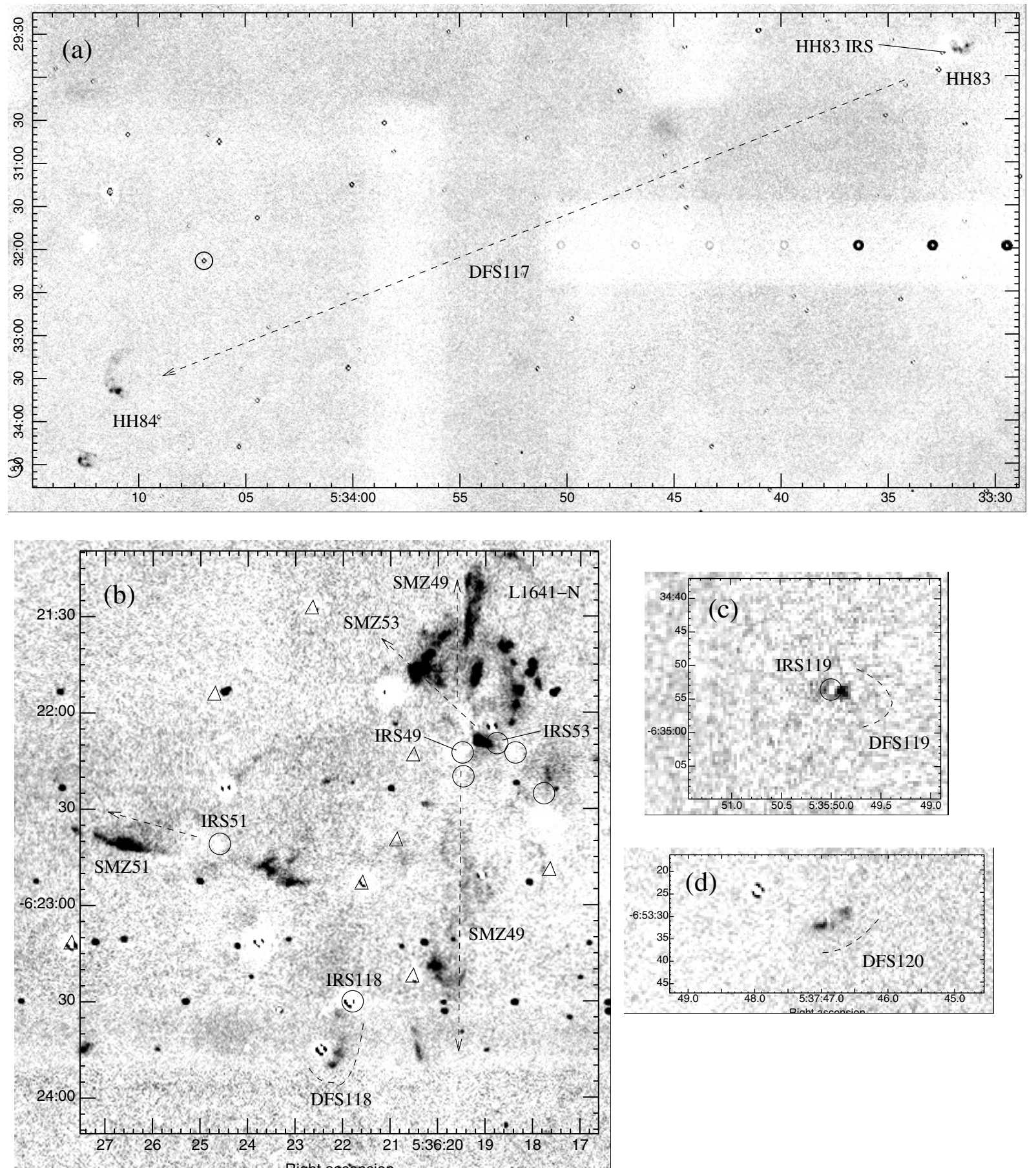

Fig. A.4. Continuum-subtracted $\mathrm{H}_{2} 2.122 \mu$ m images of DFS 117 (HH 83 and its distant counter bow-shock), DFS 118, DFS 119 and DFS 120. Protostars and disc-excess sources are marked.

\section{A.3.3. $\mathrm{HH} 1 / 2, \mathrm{SMZ} 59$ to SMZ 65, DFS 119, DFS 121, and DFS 124}

SMZ 59 is the well-known molecular outflow V380 Ori-NE (e.g. Davis et al. 2000); the source is associated with a compact molecular core and appears as a point source at $4.5 \mu \mathrm{m}$ and $24 \mu \mathrm{m}$. However, it was rejected from the Spitzer YSO catalogues as a possible extragalactic contamination because it is rather faint. Even so, its colours are consistent with those of a protostar.

The outflows in the vicinity of HH 1/2 (SMZ 60-SMZ 65) are well documented (e.g. Hester et al. 1998; Reipurth et al. 2000). There are a number of Spitzer YSOs and dust cores in NGC 1999 (near HH 1/2); most of the SMZ flows likely have candidate driving sources. The VLA source known to drive $\mathrm{HH}$ 1/2 (SMZ 64) lies in the wings of a nearby, brighter source, and so does not appear in the Spitzer YSO catalogues. To the seven $\mathrm{H}_{2}$ flows identified by Sta02 in this region we add DFS 121 (HH 36), which is probably driven by the Spitzer protostar just to the south of the embedded HH 1/2 VLA source and its dense core (Fig. A.5a).

DFS 119 is a compact knot associated with a Spitzer protostar, (Fig. A.4c). DFS 124 is a bow-shaped feature $\sim 6^{\prime}$ south of HH 2 (Fig. A.5c). It has a PM consistent with IRS 124 being the driving source. 
C. J. Davis et al.: A census of molecular hydrogen outflows and their sources along the Orion A molecular ridge, Online Material $p 6$
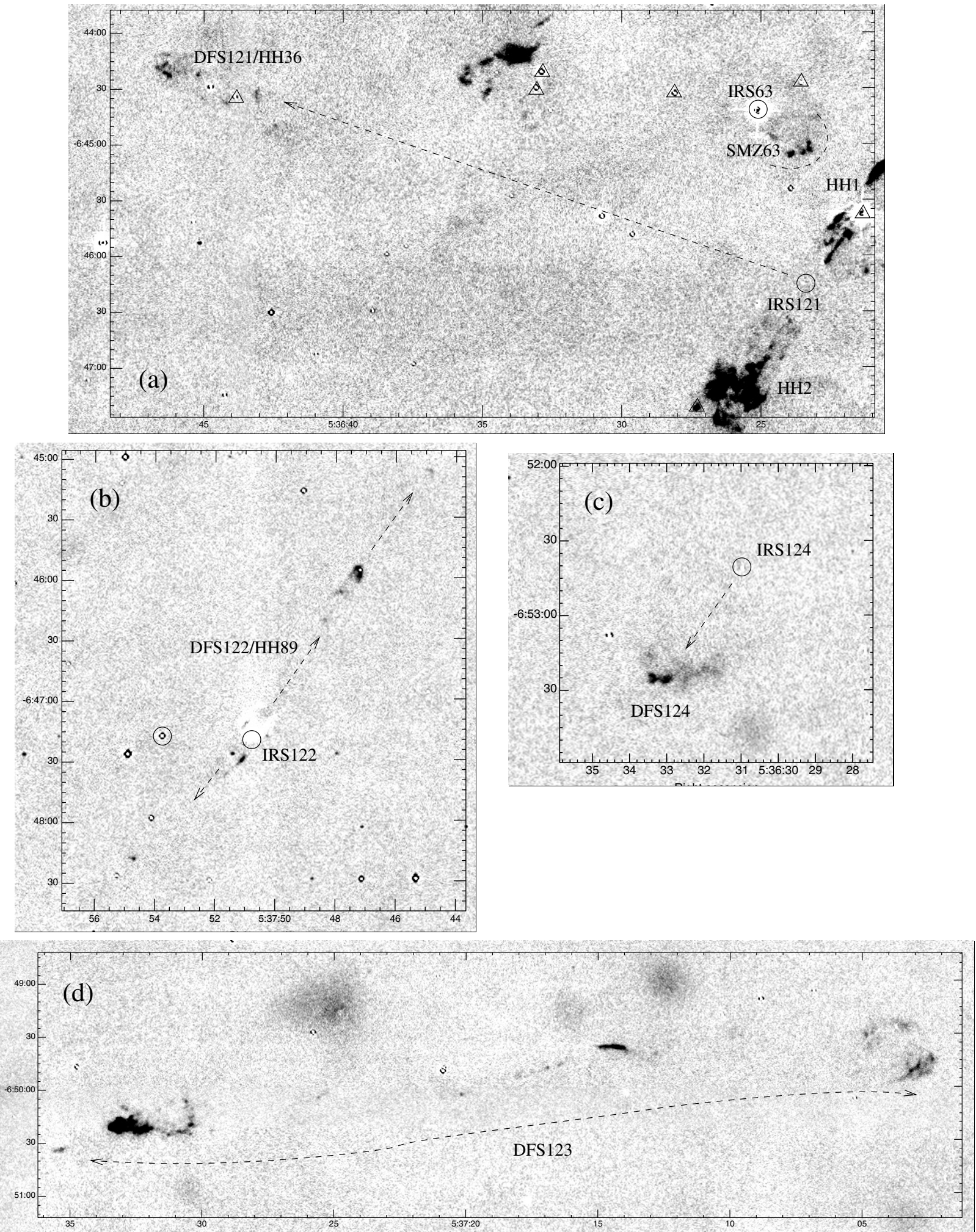

Fig. A.5. Continuum-subtracted $\mathrm{H}_{2} 2.122 \mu$ m images of DFS 121 (HH 36), DFS 122 (HH 89), DFS 123 and DFS 124. Protostars and disc-excess sources are marked.

\section{A.3.4. DFS 120, DFS 122, DFS 123, DFS 125 and DFS 126}

Five new $\mathrm{H}_{2}$ flows are identified to the southeast and southwest of HH $1 / 2$. DFS 120 is a faint compact feature with no Spitzer source (Fig. A.4d). DFS 122 and DFS 125 are associated with HH 89 and HH 127, respectively (Figs. A.5b and A.6a); the former is driven by a nebulous Spitzer protostar, IRS 122
DFS 123 is a remarkable new $\mathrm{H}_{2}$ flow, though it has no $\mathrm{HH}$ counterpart nor candidate driving source (Fig. A.5d). DFS 123 extends over at least 8.5'.

The bright $\mathrm{H}_{2}$ bow in DFS 126 (Fig. A.6d; see also Fig. 11) could be an extension of the HH 38/43/68 flow, although the orientation of the bow shock and the location of the protostar labelled IRS 126 suggests the presence of a new flow. 
C. J. Davis et al.: A census of molecular hydrogen outflows and their sources along the Orion A molecular ridge, Online Material $p 7$
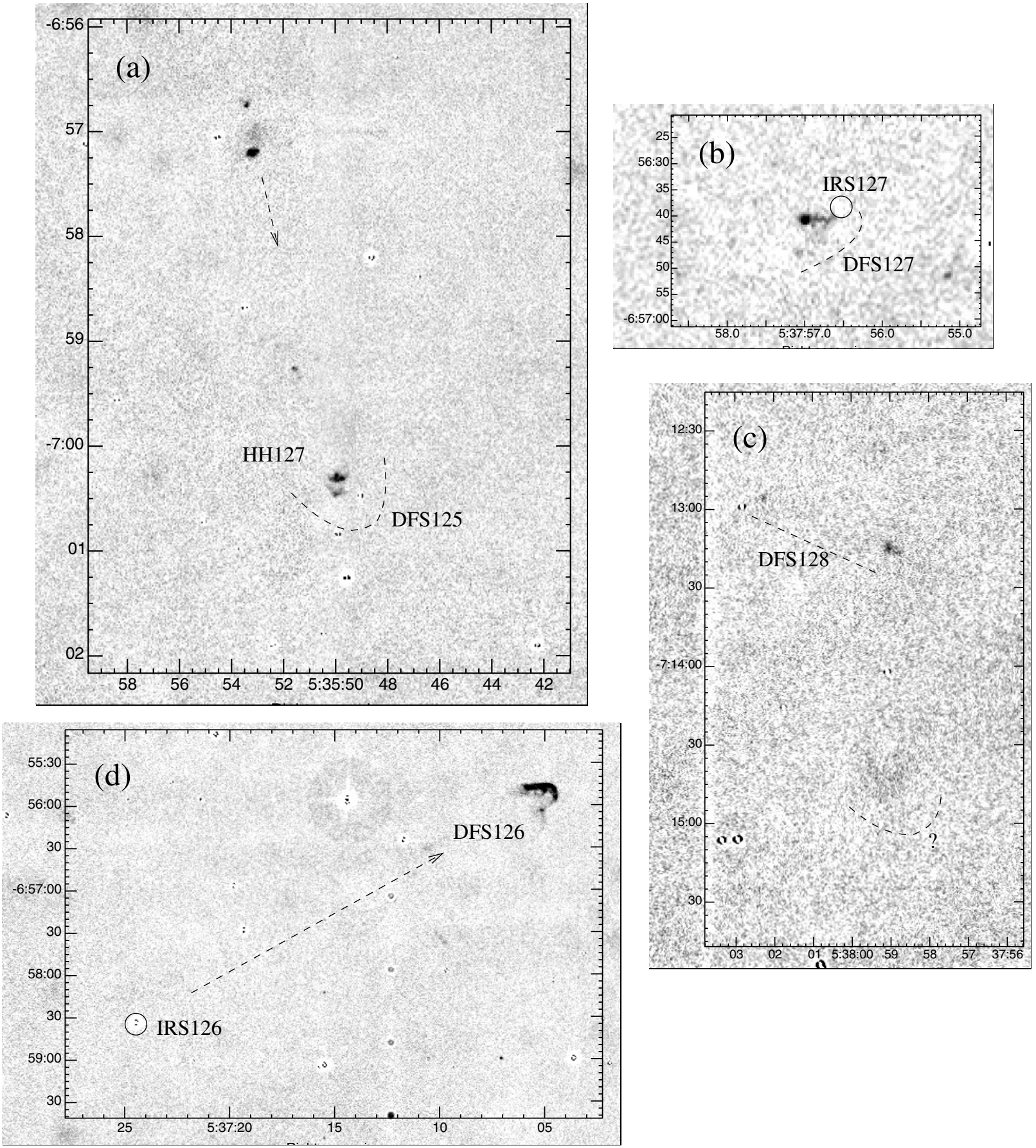

Fig. A.6. Continuum-subtracted $\mathrm{H}_{2} 2.122 \mu$ m images of DFS 125 (HH 127), DFS 126, DFS 127 and DFS 128. Protostars and disc-excess sources are marked.

Unfortunately these five outflows are beyond the bounds of the MAMBO and SCUBA observations.

\section{A.4. $L 1641-C\left(-7.0^{\circ}>\delta>-7.5^{\circ}\right)$}

\section{A.4.1. SMZ 66 and HH 38/43/64 (SMZ 67 and SMZ 68)}

In $\mathrm{H}_{2}$ emission we detect only one jet associated with the young cluster L 1641-C (Fig. 10). Based on morphology alone, SMZ 66 is probably driven by the protostar labelled IRS 66, which is the only source in the region that is associated with a sizable dust core.
SMZ 67 is better known as the parsec-scale flow HH 38/43/64 (Fig. 11). The source of this flow, and the neighbouring jet SMZ 68, are both retrieved from the Spitzer data. IRS 67 and IRS 68 are also associated with molecular cores. If we include DFS 126 in the HH 38/43/64 flow (though see the comments in the previous section), the total length of this system is a remarkable $25^{\prime}$ ( $3.3 \mathrm{pc}$ at a distance of $450 \mathrm{pc}$ ).

\section{A.4.2. SMZ 69, SMZ 70, DFS 127, DFS 128 and DFS 129}

Approximately $6^{\prime}-8^{\prime}$ south of L 1641-C, SMZ 69, SMZ 70, and DFS 129 (previously identified as the eastern end of SMZ 69; see Fig. A.7d) are driven by three of the young stars in the region. 
C. J. Davis et al.: A census of molecular hydrogen outflows and their sources along the Orion A molecular ridge, Online Material $p 8$
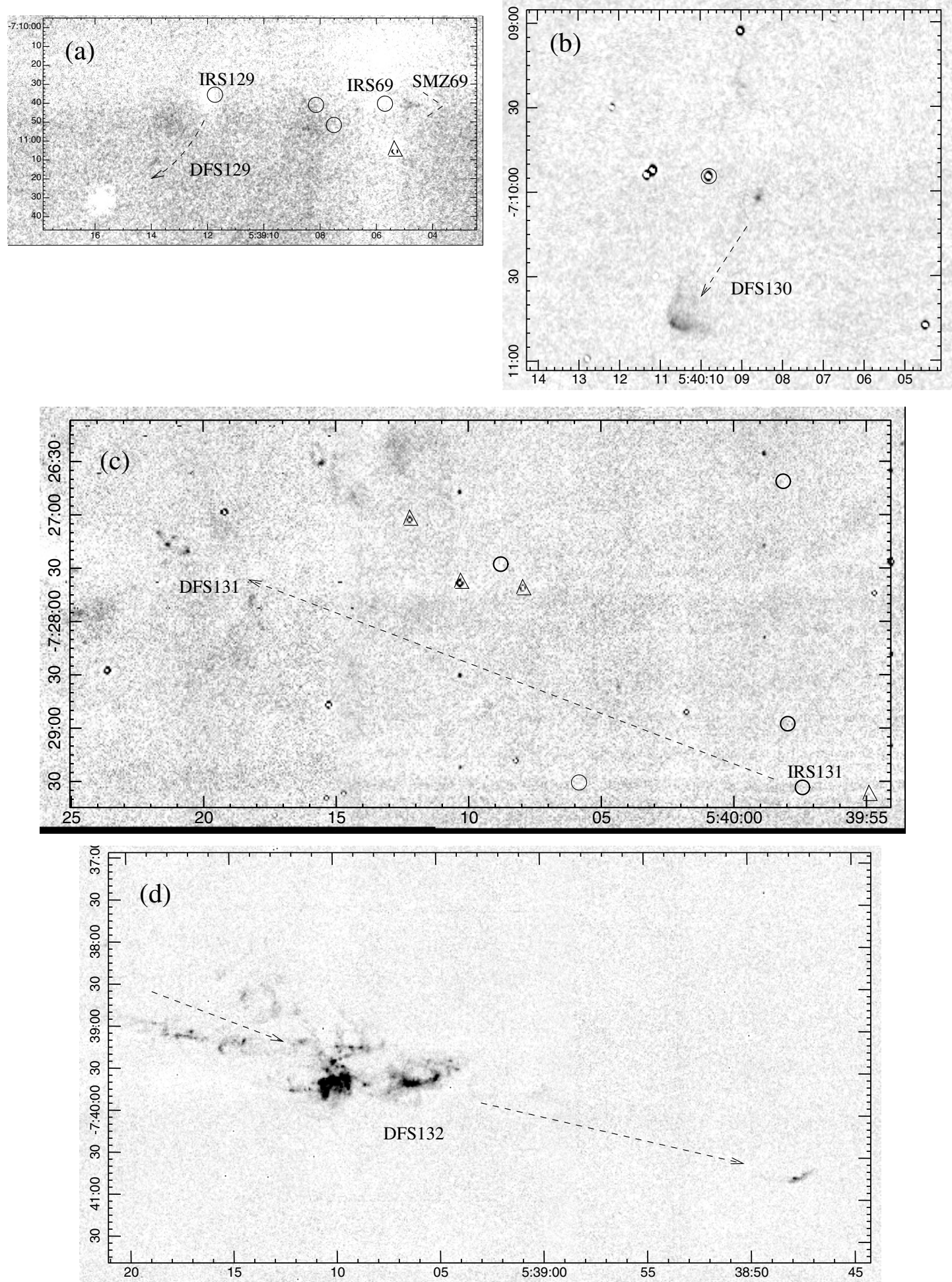

Fig. A.7. Continuum-subtracted $\mathrm{H}_{2} 2.122 \mu$ m images of DFS 129, DFS 130, DFS 131 and DFS 132. Protostars and disc-excess sources are marked.

DFS 127 and DFS 128 are faint, compact $\mathrm{H}_{2}$ features off the main L 1641 axis (Figs. A.6b and c). DFS 127 is associated with a Spitzer protostar, IRS 127.

\section{A.4.3. SMZ 71 to SMZ 76 and DFS 130 to DFS 133}

SMZ 71 is a faint, knotty $\mathrm{H}_{2}$ jet $\sim 10^{\prime}$ northwest of Haro 4-255 (midway between L 1641-C and L 1641-S). The central source is recovered by Spitzer.
SMZ 72 and SMZ 73 are orthogonal outflows in the vicinity of Haro 4-255 (Fig. 12). They appear to be associated with HH objects 469 and 470, respectively (Aspin \& Reipurth 2000). Two candidate sources for SMZ 72/HH 469 are detected in the Spitzer data. These coincide with a dense molecular core. However, although IRS 72a is better aligned with the peak of the $1200 \mu \mathrm{m}$ emission, IRS 72b coincides with the only bright VLA source in the region, Haro 4-255 FIR (Anglada et al. 1998). We therefore associate IRS 72b with SMZ 72, though add that both 
sources may contribute to the excitation of the $\mathrm{HH} / \mathrm{H}_{2}$ features. SMZ 73/HH 470 is almost certainly driven by the bright $K$-band source/T Tauri star Haro 4-255. Haro 4-255 is associated with a compact optical jet (Aspin \& Reipurth 2000) and faint $1200 \mu \mathrm{m}$ dust continuum emission.

SMZ 74 and SMZ 75 are compact groups of $\mathrm{H}_{2}$ knots $6^{\prime}-8^{\prime}$ north of Re50. Their morphologies and PMs suggest that they are powered by one of a number of Spitzer protostars located $7^{\prime}-8^{\prime}$ to the southwest, although precise associations are difficult to make.

SMZ 76 is a spectacular curving $\mathrm{H}_{2}$ flow a few arcminutes north of $\mathrm{Re} 50$. The PMs of the two elongated arcs of $\mathrm{H}_{2}$ emission that comprise SMZ 76 are generally directed towards the east, again as one would expect if they were being driven by a source situated to the west or west-southwest. Stanke et al. (2000) discuss this region in detail, and identify a millimetre peak (which they label L 1641-S3 MMS1) as the location of the likely source. This embedded source was detected by MAMBO and Spitzer and is identified as a candidate protostar (IRS 76).

Roughly $20^{\prime}$ southwest of SMZ 76 we discover a similarly large $\mathrm{H}_{2}$ feature, DFS 132 (Figs. 12 and A.7d). The overall morphology of this feature suggests motion away from IRS 76. If SMZ 76 and DFS 132 are part of the same flow, then the total length is at least $35^{\prime}$ ( $\left.4.6 \mathrm{pc}\right)$. However, without proper motion data or, preferably, a large-scale (sub)millimetre outflow map in $\mathrm{CO}$, this association remains uncertain.

DFS 133 is a faint, twisting chain of $\mathrm{H}_{2}$ emission features between SMZ 76 and DFS 132 (Fig. A.8a). First noted by Stanke (2000), it is possibly driven by the protostar/dust core to the north labelled IRS 133.

Two additional $\mathrm{H}_{2}$ flows, DFS 130 and DFS 131, are presented in Figs. A.7b and c. DFS 130 is a well-defined bow shock with no identified driving source. DFS 131 is a chain of knots to the south of SMZ 76 that may represent an independent system. The PMs of these knots are well aligned along an axis that links DFS 131 with a MAMBO dust core and its associated Spitzer protostar IRS 131.

\section{A.5. $L$ 1641-S $\left(-7.5^{\circ}>\delta>-8.3^{\circ}\right)$}

\section{A.5.1. DFS 134 to DFS 142}

Finally, the region at the southern end of L 1641 is abundant with Spitzer-identified young stars and newly discovered $\mathrm{H}_{2}$ flows. We identify nine possible outflows, most of which have candidate Spitzer sources (note that the MAMBO and SCUBA observations do not extend this far south in L 1641).

DFS 138 is the most spectacular flow, comprising a collimated, bipolar jet and features that delineate sweeping bow shocks (Fig. A.9a). The outflow extends over 13.4' (1.8 pc) and appears to be driven by a Spitzer protostar.

Sources are also identified for DFS 136, DFS 137, DFS 141 and DFS 143 (see Figs. A.8 and A.10). DFS 135 is a collimated jet and bow shock that seems to be driven by a bright, near-IR source, though this star is not in our list of Spitzer disc sources or protostars (Fig. A.8c). DFS 139 is a faint, curving wisp of line emission - possibly a collimated jet (Fig. A.9b). DFS 143 is an extremely faint flow, though it is associated with a protostar (Fig. A.10a).

The remaining $\mathrm{H}_{2}$ features in the region are: the compact arcs that comprise DFS 134, the knotty feature DFS 142, and the parallel "fingers" of emission collectively labelled DFS 140 (Figs. A.8-A.10). However, none of these objects have an obvious Spitzer source. 
C. J. Davis et al.: A census of molecular hydrogen outflows and their sources along the Orion A molecular ridge, Online Material p 10
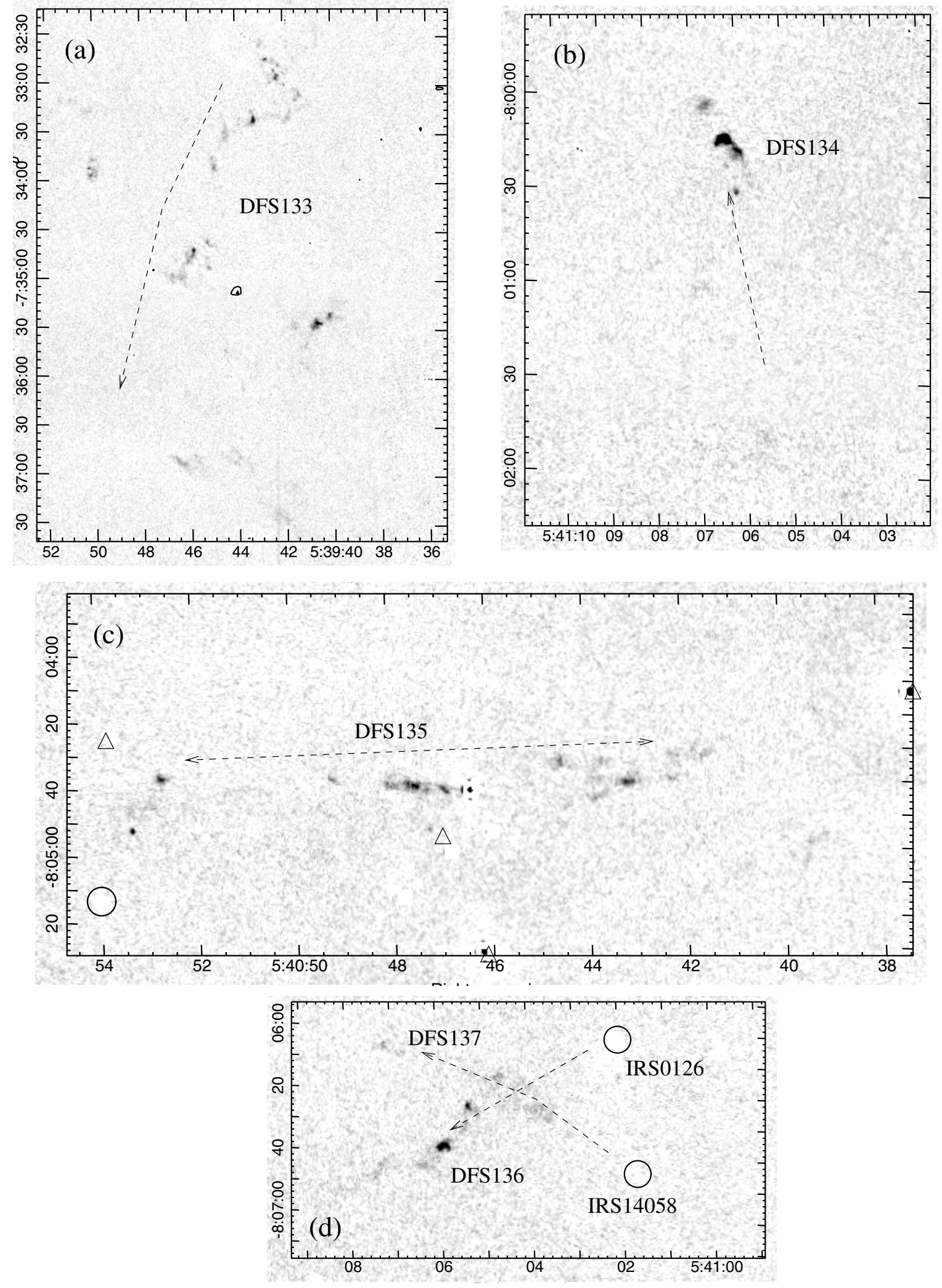

Fig. A.8. Continuum-subtracted $\mathrm{H}_{2} 2.122 \mu$ m images of DFS 133 to DFS 137. Protostars and disc-excess sources are marked. 
C. J. Davis et al.: A census of molecular hydrogen outflows and their sources along the Orion A molecular ridge, Online Material p 11
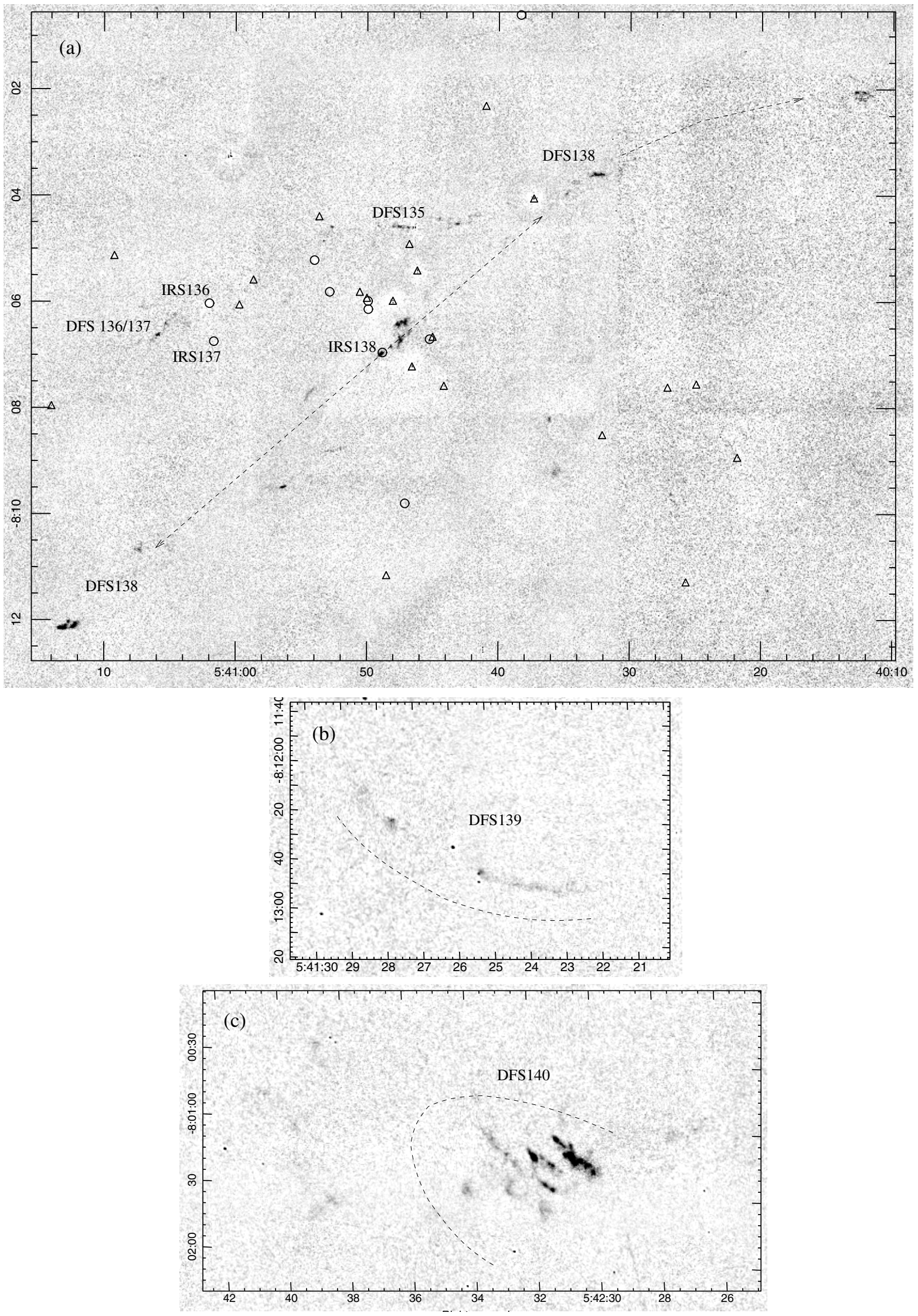

Fig. A.9. Continuum-subtracted $\mathrm{H}_{2} 2.122 \mu$ m images of DFS 138, DFS 139 and DFS 140. Protostars and disc-excess sources are marked. 
C. J. Davis et al.: A census of molecular hydrogen outflows and their sources along the Orion A molecular ridge, Online Material $p$ 12
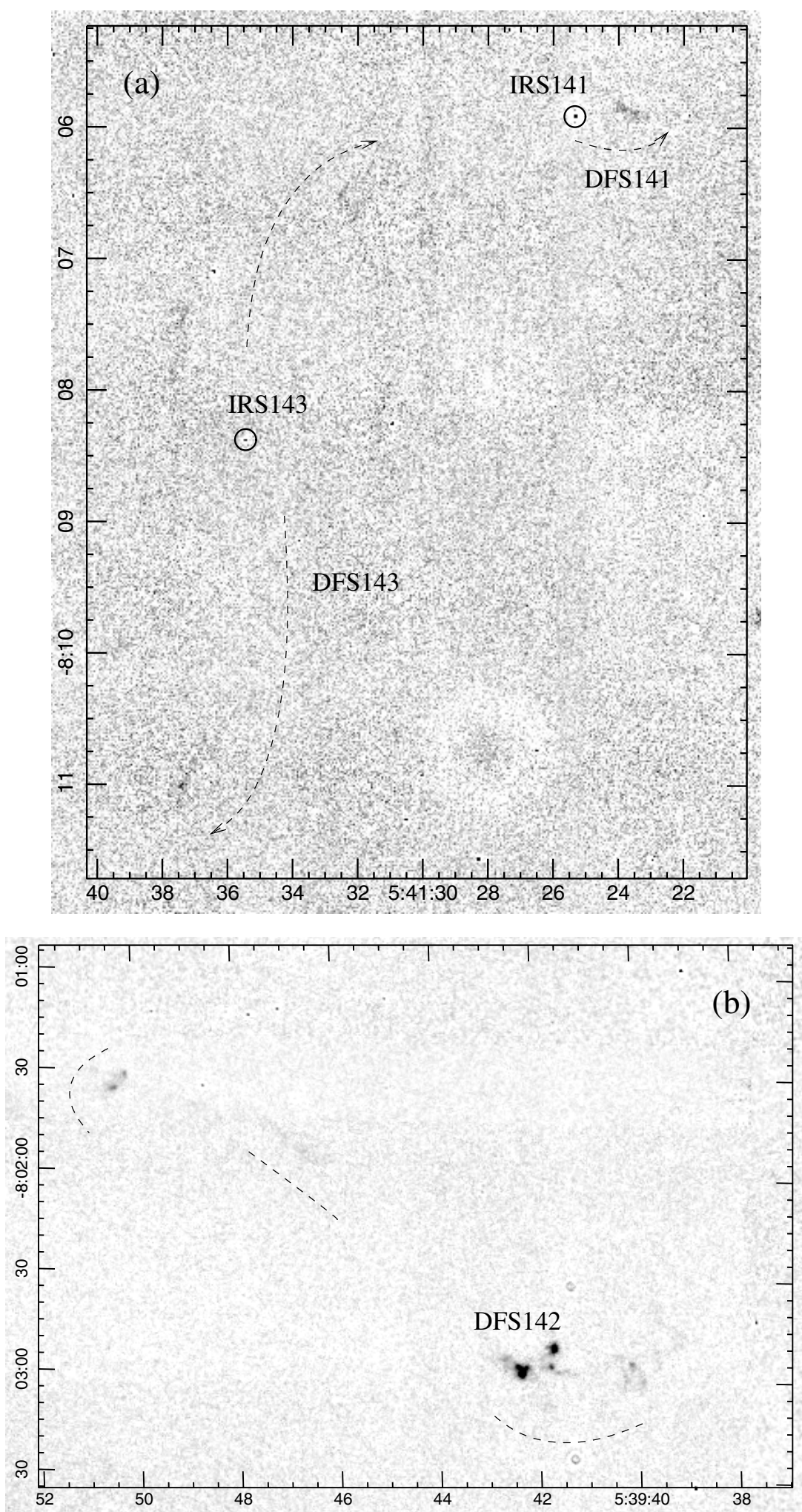

Fig. A.10. Continuum-subtracted $\mathrm{H}_{2} 2.122 \mu \mathrm{m}$ images of DFS 141, DFS 142 and DFS 143. Protostars and disc-excess sources are marked. 


\section{Appendix B: Proper motion measurements}

The proper motions (PMs) of selected bright $\mathrm{H}_{2}$ knots have been measured. PMs are listed in Table B.1, where we use the knot nomenclature of $\mathrm{Sta0} 2$ when referring to features within each SMZ or DFS outflow. An extensive catalogue of images, with the individual features labelled, is also available from Stanke (2000); an on-line version is available at http://www.eso. org/ tstanke/thesis/.

Although we did not measure PMs for every outflow common to the Sta02 data and the new WFCAM images, the number of objects analysed is considerable and therefore the sample represents a relatively unbiased survey of $\mathrm{H}_{2}$ knot PMs in low mass protostellar outflows.

The first and second epoch images were obtained with different instruments. Hence, the first epoch images were converted to the same pixel size as the WFCAM data; all results are therefore with respect to the WFCAM pixel scale of $0.2^{\prime \prime}$.

The PMs of knots and stars were measured using a crosscorrelation method. For each region the first epoch image was registered with the corresponding second epoch image using a third-order polynomial fit to all detected stars in the field (the IRAF routines geomap and geotran were employed). Typical root-mean-square (rms) values for the fit were below 0.5 pixels, i.e. $<0.1^{\prime \prime}$. The first epoch image was then shifted relative to the second epoch image in steps of 0.25 pixels $\left(0.05^{\prime \prime}\right)$ about a $5 \times 5$ pixel grid. At each position the total flux in a rectangular box surrounding a star or knot was determined from the product of the second and shifted-first epoch images. This total flux was recorded as the cross-correlation coefficient for a given star or knot at each relative shift between the images. The sizes of boxes around target features were manually determined in order to enclose the entire flux from each object. The process yields a set of correlation coefficients for each object $(c c f=f(\operatorname{shift} x$, shift $y))$. We then determined manually the position of the maximum correlation coefficient using the center/moment command in the European Southern Observatory's MIDAS package. This position was recorded as the PM of the individual object. Finally, to determine the PM of the outflow knots, we subtracted the average PM of adjacent field stars. This ensures that any systematic effects (i.e. problems with the mosaicing in the 1 st epoch images) were minimised.

The random errors, i.e. the uncertainties in the determination of the individual PMs of stars and bright knots, is very low. For reasonably bright stars the center/moment command determined the PM to an accuracy of up to $1 / 30$ th of a pixel $\left(0.007^{\prime \prime}\right)$. Even for faint stars, accuracies better than 0.1 pixels were attained. For extended and low signal-to-noise knots, the uncertainties can be slightly larger ( 0.1 pixel; $\left.0.02^{\prime \prime}\right)$. In situations where the position of the maximum correlation coefficient was uncertain, the center/moment command could not be used. These objects are flagged with status $F=2$ in Table B.1, and should be viewed with caution.

The random motions of field stars represent the most significant source of error. We found that in most cases, between the two epochs, the PMs of the stars in our images was of the order of 0.35 pixels $\left(0.07^{\prime \prime}\right)$, i.e. much larger than the random errors associated with our method. With our epoch difference of 8-9 years, the PM of the field stars translates to almost $0.01^{\prime \prime} /$ year (a typical value for field star PMs). The errors for the measured PMs of outflow knots therefore depends on the number of adjacent field stars used as a control sample, and the assumption that on average those stars move in random directions. We adopt a $1 \sigma$ error for the PMs of outflow features of $\left.0.07^{\prime \prime} / \sqrt{(} N\right)$ (the contribution from the field stars) added in quadrature with $0.02^{\prime \prime}$ (the contribution from our method). Since a similar number of field stars were used in each region (between 8 and 20), the $3 \sigma$ error is typically $0.09^{\prime \prime}\left( \pm 0.015^{\prime \prime}\right)$ or $21 \mathrm{~km} \mathrm{~s}^{-1}\left( \pm 3 \mathrm{~km} \mathrm{~s}^{-1}\right)$.

The above assumes that $\mathrm{H}_{2}$ knot brightnesses and morphologies do not change between first and second-epoch observations: in many features this is clearly not the case. The contribution to the actual PM is difficult to determine generally, since it depends on the percentage increase of flux from the knot and where it occurs. Also, adjacent objects can influence the PM measurements, when the shifting of the first epoch image moves a knot onto a neighbouring object. If this object is brighter, then the actual distance to the neighbouring object is measured as the PM. We have therefore not measured PMs for targets that are within $2^{\prime \prime}$ of other objects. Finally, a gradient in the background emission can also lead to a measured PM which is not real. This can occur in regions with spatially variable nebulous emission or close to very bright stars. Hence, any PM pointing along the slope of the background emission should be treated with caution.

Below we briefly interpret the results outlined in Table B.1. We split the $\mathrm{H}_{2}$ outflows up into five sections, B.1 to B.5; PM vectors are drawn onto contour plots of most of the outflows in the corresponding five figures, Figs. B.1-B.5 (note that figures are not presented for all $\mathrm{H}_{2}$ flows).

\section{B.1. $O M C 3$}

The PMs of SMZ 8 to SMZ 18 are displayed in Fig. B.1.

\section{B.1.1. SMZ 8}

1-14A is the main $\mathrm{H}_{2}$ feature in this object, which we resolve into four peaks (A, An, A-s and Aw). However, the tangential velocities of these four peaks are randomly orientated so no overall PM is identified. 1-14B is a faint arc $\sim 50^{\prime \prime}$ southwest of A which appears to be moving eastward.

\section{B.1.2. SMZ 9}

$1-18 \mathrm{~A}$ and $\mathrm{B}$ are the brightest of many $\mathrm{H}_{2}$ knots (A-J) that comprise the SMZ 9 outflow. A and B are bow shocks at the western end of the flow that do indeed move westward. Features D-J are bright knots within bows midway between bows 1-18A/B and the proposed source, IRS 9. Although there is some scatter in PMs and velocities are low, all again appear to be moving westward, as expected.

\section{B.1.3. SMZ 10}

On morphological grounds $1-16 \mathrm{~A}$ and $1-16 \mathrm{~B}+\mathrm{C}$ seem to be sections of bow shocks driven by IRS 10 toward the northeast; both knots are associated with a bipolar CO outflow. The modest PMs displayed in Fig. B.1 do not support this interpretation, however.

\section{B.1.4. SMZ $11 / 13$}

This $\mathrm{H}_{2}$ flow comprises a north-south twisting chain of knots $1-22 \mathrm{~A}$ to $1-22 \mathrm{D}$ that extends $\sim 1^{\prime}$ northward to include knot 1-19A. 1-22A and 1-22B are the brightest features with the best-defined PMs; these move southward away from the protostar IRS 11, located midway between A and D. 1-22 C and D also move roughly southward, although these PMs are relatively 
C. J. Davis et al.: A census of molecular hydrogen outflows and their sources along the Orion A molecular ridge, Online Material p 14

Table B.1. Proper Motions of $\mathrm{H}_{2}$ features.

\begin{tabular}{|c|c|c|c|c|c|c|c|c|c|c|}
\hline$\overline{\text { Flow }}$ & $\overline{\text { Knot }}$ & $\begin{array}{l}\text { RA } \\
(2000)\end{array}$ & $\begin{array}{l}\text { Dec } \\
(2000)\end{array}$ & $\begin{array}{r}\text { shift- } x \\
\text { (pix) }\end{array}$ & $\begin{array}{r}\text { shift- } y \\
\text { (pix) }\end{array}$ & $\begin{array}{l}\text { shift } \\
\text { (pix) }\end{array}$ & $\begin{array}{r}\text { shift } \\
(\operatorname{arcsec})\end{array}$ & $\begin{array}{r}\mathrm{Vel}^{a} \\
\left(\mathrm{~km} \mathrm{~s}^{-1}\right)\end{array}$ & $\begin{array}{r}\mathrm{PA}^{b} \\
(\mathrm{deg})\end{array}$ & $\overline{\overline{\mathrm{F}^{c}}}$ \\
\hline SMZ 8* & $1-14 \mathrm{~A}$ & $5: 35: 42.1$ & $-5: 04: 40$ & -1.804 & 0.285 & 1.83 & 0.37 & 83.6 & 81.0 & 0 \\
\hline " & $1-14 \mathrm{An}$ & $5: 35: 42.1$ & $-5: 04: 35$ & 1.018 & 0.441 & 1.11 & 0.22 & 50.7 & -66.6 & 2 \\
\hline " & $1-14 \mathrm{As}$ & $5: 35: 41.9$ & $-5: 04: 43$ & 0.268 & -0.684 & 0.73 & 0.15 & 33.3 & -158.6 & 2 \\
\hline " & $1-14 B$ & $5: 35: 44.2$ & $-5: 05: 04$ & -1.173 & 0.058 & 1.17 & 0.23 & 53.4 & 87.2 & 0 \\
\hline SMZ 9 & $1-18 \mathrm{~A}$ & $5: 35: 15.5$ & $-5: 06: 12$ & 1.432 & 0.375 & 1.48 & 0.30 & 67.6 & -75.3 & 0 \\
\hline " & $1-18 B$ & $5: 35: 16.3$ & $-5: 06: 01$ & 1.690 & 0.984 & 1.96 & 0.39 & 89.5 & -59.8 & 0 \\
\hline " & $1-18 \mathrm{C}$ & $5: 35: 18.3$ & $-5: 05: 50$ & 0.243 & 0.868 & 0.90 & 0.18 & 41.1 & -15.6 & 0 \\
\hline " & $1-18 E$ & $5: 35: 22.8$ & $-5: 05: 49$ & 0.944 & 0.761 & 1.21 & 0.24 & 55.3 & -51.1 & 0 \\
\hline " & $1-18 \mathrm{~F}$ & $5: 35: 23.9$ & $-5: 05: 54$ & 3.089 & -0.100 & 3.09 & 0.62 & 141.1 & -91.9 & 0 \\
\hline " & $1-18 \mathrm{G}$ & $5: 35: 25.5$ & $-5: 05: 51$ & -0.073 & 0.458 & 0.47 & 0.09 & 21.5 & 9.1 & 0 \\
\hline " & $1-18 \mathrm{~J}$ & $5: 35: 23.3$ & $-5: 05: 53$ & 0.927 & 0.334 & 0.98 & 0.20 & 44.8 & -70.2 & 2 \\
\hline SMZ 10 & $1-16 A$ & $5: 35: 31.5$ & $-5: 04: 39$ & -1.222 & -0.356 & 1.27 & 0.25 & 58.0 & -253.8 & 0 \\
\hline " & $1-16 B+C$ & $5: 35: 31.2$ & $-5: 04: 37$ & -0.830 & -0.805 & 1.16 & 0.23 & 53.0 & -225.9 & 0 \\
\hline SMZ 11/13 & $1-19 A$ & $5: 35: 23.7$ & $-5: 06: 00$ & -0.680 & 1.176 & 1.36 & 0.27 & 62.1 & 30.0 & 0 \\
\hline " & $1-22 \mathrm{~A}$ & $5: 35: 22.6$ & $-5: 07: 17$ & 0.325 & -1.710 & 1.74 & 0.35 & 79.5 & -169.2 & 0 \\
\hline " & $1-22 B$ & $5: 35: 22.2$ & $-5: 07: 25$ & 0.616 & -0.670 & 0.91 & 0.18 & 41.6 & -137.4 & 0 \\
\hline " & $1-22 C$ & $5: 35: 23.5$ & $-5: 06: 41$ & 0.681 & -0.181 & 0.70 & 0.14 & 32.0 & -104.9 & 0 \\
\hline " & $1-22 D$ & $5: 35: 23.2$ & $-5: 06: 56$ & 0.059 & -0.735 & 0.74 & 0.15 & 33.8 & -175.4 & 0 \\
\hline SMZ 14 & $1-20 \mathrm{~A}$ & $5: 35: 31.4$ & $-5: 06: 23$ & 0.643 & 1.245 & 1.40 & 0.28 & 63.9 & -27.3 & 0 \\
\hline " & $1-20 B$ & $5: 35: 32.2$ & $-5: 06: 18$ & 0.061 & 1.510 & 1.51 & 0.30 & 69.0 & -2.3 & 0 \\
\hline SMZ 17/18 & $1-27 \mathrm{~A}$ & $5: 35: 30.0$ & $-5: 08: 20$ & -1.308 & 1.746 & 2.18 & 0.44 & 99.6 & 36.8 & 0 \\
\hline$"$ & $1-27 B$ & $5: 35: 29.6$ & $-5: 08: 57$ & -2.476 & 0.412 & 2.51 & 0.50 & 114.6 & 80.6 & 0 \\
\hline " & $1-27 C+D$ & $5: 35: 28.3$ & $-5: 08: 51$ & -0.304 & 0.429 & 0.53 & 0.11 & 24.2 & 35.3 & 2 \\
\hline " & $1-27 E$ & $5: 35: 28.5$ & $-5: 09: 02$ & -1.054 & -0.571 & 1.20 & 0.24 & 54.8 & -241.6 & 2 \\
\hline " & $1-27 \mathrm{~F}$ & $5: 35: 30.1$ & $-5: 08: 35$ & -1.778 & -1.184 & 2.14 & 0.43 & 97.7 & -236.3 & 0 \\
\hline SMZ 21/22 & $1-37 \mathrm{~A}$ & $5: 35: 27.3$ & $-5: 11: 49$ & -0.102 & -1.532 & 1.54 & 0.31 & 70.3 & -183.8 & 0 \\
\hline " & $1-37 \mathrm{~B}$ & $5: 35: 26.9$ & $-5: 12: 19$ & 0.343 & -0.954 & 1.01 & 0.20 & 46.1 & -160.2 & 0 \\
\hline " & $1-37 \mathrm{C}$ & $5: 35: 27.4$ & $-5: 12: 48$ & 0.513 & -2.694 & 2.74 & 0.55 & 125.1 & -169.2 & 0 \\
\hline SMZ 23 & $1-38 \mathrm{~A}$ & $5: 35: 20.1$ & $-5: 14: 05$ & 1.118 & -1.688 & 2.02 & 0.40 & 92.3 & -146.5 & 2 \\
\hline " & $1-38 B$ & $5: 35: 21.6$ & $-5: 12: 55$ & -2.013 & 0.055 & 2.01 & 0.40 & 91.8 & 88.4 & 0 \\
\hline " & $1-38 D$ & $5: 35: 22.0$ & $-5: 12: 36$ & 0.543 & 0.447 & 0.70 & 0.14 & 32.0 & -50.5 & 0 \\
\hline " & $1-38 \mathrm{E}$ & $5: 35: 23.0$ & $-5: 11: 45$ & 0.993 & 1.187 & 1.55 & 0.31 & 70.8 & -39.9 & 0 \\
\hline SMZ 24 & $1-40 \mathrm{~A}$ & $5: 35: 23.3$ & $-5: 12: 00$ & 1.268 & -0.779 & 1.49 & 0.30 & 68.0 & -121.6 & 0 \\
\hline " & $1-40 \mathrm{~A} 1$ & $5: 35: 22.5$ & $-5: 12: 10$ & 1.855 & -0.914 & 2.07 & 0.41 & 99.2 & -116.2 & 0 \\
\hline " & $1-40 B$ & $5: 35: 18.4$ & $-5: 12: 42$ & 2.473 & -1.124 & 2.72 & 0.54 & 124.2 & -114.4 & 0 \\
\hline$"$ & $1-40 \mathrm{C}$ & $5: 35: 17.5$ & $-5: 12: 45$ & 1.018 & -1.646 & 1.94 & 0.39 & 88.6 & -148.3 & 0 \\
\hline SMZ 25 & $1-32 \mathrm{~A}$ & $5: 35: 16.2$ & $-5: 10: 25$ & -0.316 & -0.571 & 0.65 & 0.13 & 29.7 & -209.0 & 0 \\
\hline " & $1-32 B$ & $5: 35: 16.5$ & $-5: 10: 53$ & -0.055 & 1.006 & 1.01 & 0.20 & 46.1 & 3.1 & 0 \\
\hline " & $1-32 C$ & $5: 35: 16.2$ & $-5: 09: 51$ & -1.189 & -0.001 & 1.19 & 0.24 & 54.3 & -270.0 & 0 \\
\hline " & $1-39 A$ & $5: 35: 18.9$ & $-5: 11: 40$ & 0.228 & 0.683 & 0.72 & 0.14 & 32.9 & -18.5 & 2 \\
\hline$"$ & $1-39 B$ & $5: 35: 19.0$ & $-5: 11: 49$ & 0.353 & -0.317 & 0.47 & 0.09 & 21.5 & -131.9 & 2 \\
\hline SMZ 31 & $3-9 A$ & $5: 34: 35.3$ & $-5: 39: 59$ & 0.198 & 0.675 & 0.70 & 0.14 & 37.9 & -16.3 & 2 \\
\hline " & $3-9 \mathrm{~B}$ & $5: 34: 34.9$ & $-5: 39: 44$ & 0.173 & 3.008 & 3.01 & 0.60 & 163.1 & -3.3 & 0 \\
\hline SMZ 32 & $3-8 n$ & $5: 35: 11.5$ & $-5: 39: 28$ & 0.800 & -1.129 & 1.38 & 0.28 & 74.8 & -144.7 & 0 \\
\hline " & $3-8 \mathrm{~s}$ & $5: 35: 11.3$ & $-5: 39: 39$ & -0.514 & 0.690 & 0.86 & 0.17 & 46.6 & 36.7 & 0 \\
\hline " & $3-10$ & $5: 35: 11.3$ & $-5: 40: 13$ & -0.283 & 1.399 & 1.43 & 0.29 & 77.5 & 11.4 & 2 \\
\hline SMZ 33 & $3-12 \mathrm{~A}$ & $5: 34: 52.8$ & $-5: 41: 52$ & 0.465 & 0.888 & 1.00 & 0.20 & 54.2 & -27.6 & 0 \\
\hline " & $3-12 C$ & $5: 34: 51.7$ & $-5: 41: 33$ & 1.904 & 0.995 & 2.15 & 0.43 & 116.5 & -62.4 & 0 \\
\hline SMZ 34 & $3-13 A$ & $5: 35: 09.7$ & $-5: 43: 45$ & -0.368 & 0.850 & 0.93 & 0.19 & 50.4 & 23.4 & 0 \\
\hline SMZ 35 & $3-14$ & $5: 34: 50.6$ & $-5: 44: 20$ & -0.635 & -0.296 & 0.70 & 0.14 & 37.9 & -245.0 & 0 \\
\hline SMZ 36 & $3-15 A$ & $5: 35: 09.9$ & $-5: 45: 05$ & 0.103 & 0.705 & 0.71 & 0.14 & 38.5 & -8.3 & 0 \\
\hline " & $3-15 B$ & $5: 35: 10.3$ & $-5: 45: 03$ & -0.819 & 0.157 & 0.83 & 0.17 & 45.0 & 79.1 & 0 \\
\hline " & $3-15 C$ & $5: 35: 10.4$ & $-5: 45: 00$ & 0.014 & -0.780 & 0.78 & 0.16 & 42.3 & -179.0 & 0 \\
\hline SMZ 37 & $3-16 A$ & $5: 35: 05.4$ & $-5: 50: 57$ & 0.504 & 0.103 & 0.51 & 0.10 & 27.6 & -78.4 & 0 \\
\hline " & $3-16 B$ & $5: 35: 06.2$ & $-5: 51: 11$ & 0.351 & -1.358 & 1.40 & 0.28 & 75.9 & -165.5 & 0 \\
\hline " & $3-17 \mathrm{~A}$ & $5: 35: 04.4$ & $-5: 52: 00$ & -0.458 & 2.166 & 2.21 & 0.44 & 119.8 & 11.9 & 0 \\
\hline " & $3-17 \mathrm{~B}$ & $5: 35: 04.6$ & $-5: 51: 51$ & -1.485 & 2.810 & 3.18 & 0.64 & 172.4 & 27.9 & 0 \\
\hline " & $3-17 \mathrm{C}$ & $5: 35: 05.3$ & $-5: 51: 26$ & -2.372 & 0.238 & 2.38 & 0.48 & 129.0 & 84.3 & 0 \\
\hline SMZ 38 & $4-1$ & $5: 35: 04.2$ & $-5: 52: 50$ & 1.463 & 3.308 & 3.62 & 0.72 & 165.3 & -23.9 & 0 \\
\hline " & $4-2 \mathrm{~A}$ & $5: 35: 05.9$ & $-5: 54: 22$ & 1.517 & 1.663 & 2.25 & 0.45 & 102.8 & -42.4 & 0 \\
\hline " & $4-2 B$ & $5: 35: 06.1$ & $-5: 54: 27$ & 0.896 & 1.416 & 1.68 & 0.34 & 76.7 & -32.3 & 0 \\
\hline "I & $4-3$ & $5: 35: 06.9$ & $-5: 54: 51$ & 0.236 & 1.921 & 1.93 & 0.39 & 88.1 & -7.0 & 0 \\
\hline
\end{tabular}


Table B.1. continued.

\begin{tabular}{|c|c|c|c|c|c|c|c|c|c|c|}
\hline Flow & Knot & $\begin{array}{l}\text { RA } \\
(2000)\end{array}$ & $\begin{array}{l}\text { Dec } \\
(2000)\end{array}$ & $\begin{array}{r}\text { shift- } x \\
\text { (pix) }\end{array}$ & $\begin{array}{r}\text { shift-y } \\
\text { (pix) }\end{array}$ & $\begin{array}{l}\text { shift } \\
\text { (pix) }\end{array}$ & $\begin{array}{r}\text { shift } \\
(\operatorname{arcsec})\end{array}$ & $\begin{array}{r}\mathrm{Vel}^{a} \\
\left(\mathrm{~km} \mathrm{~s}^{-1}\right)\end{array}$ & $\begin{array}{r}\mathrm{PA}^{b} \\
(\mathrm{deg})\end{array}$ & $q^{c}$ \\
\hline SMZ 39 & $4-4 \mathrm{~A}$ & $5: 35: 14.5$ & $-5: 56: 14$ & -2.317 & 1.811 & 2.94 & 0.59 & 134.3 & 52.0 & 0 \\
\hline " & $4-4 B$ & $5: 35: 12.5$ & $-5: 56: 51$ & -1.070 & 1.765 & 2.06 & 0.41 & 94.1 & 31.2 & 0 \\
\hline " & $4-4 \mathrm{C}$ & $5: 35: 13.8$ & $-5: 56: 29$ & 1.918 & 0.236 & 1.93 & 0.39 & 88.1 & -83.0 & 0 \\
\hline " & $4-4 \mathrm{D}$ & $5: 35: 12.7$ & $-5: 56: 27$ & -1.362 & 0.841 & 1.60 & 0.32 & 73.1 & 58.3 & U \\
\hline " & $4-5 \mathrm{~A}$ & $5: 35: 11.4$ & $-5: 57: 04$ & -1.122 & 1.074 & 1.55 & 0.31 & 70.8 & 46.3 & 0 \\
\hline " & $4-5 B$ & $5: 35: 10.4$ & $-5: 56: 52$ & -1.086 & 0.836 & 1.37 & 0.27 & 62.6 & 52.4 & 0 \\
\hline " & $4-5 \mathrm{C}$ & $5: 35: 10.7$ & $-5: 56: 59$ & -2.596 & -1.399 & 2.95 & 0.59 & 134.7 & -241.7 & 0 \\
\hline$"$ & $4-5 \mathrm{D}$ & $5: 35: 11.2$ & $-5: 56: 50$ & -0.303 & 1.248 & 1.28 & 0.26 & 58.5 & 13.6 & 0 \\
\hline " & $4-6$ & $5: 35: 13.1$ & $-5: 57: 11$ & -0.114 & 1.364 & 1.37 & 0.27 & 65.6 & 4.8 & 0 \\
\hline " & $4-7$ & $5: 35: 10.7$ & $-5: 57: 37$ & 1.588 & -0.977 & 1.86 & 0.37 & 84.9 & -121.6 & 0 \\
\hline SMZ 40 & $4-9 A+B$ & $5: 35: 37.3$ & $-6: 02: 30$ & 1.156 & -1.848 & 2.18 & 0.44 & 104.4 & -148.0 & 0 \\
\hline " & $4-9 A$ & $5: 35: 37.2$ & $-6: 02: 30$ & -0.618 & 0.261 & 0.67 & 0.13 & 30.6 & 67.1 & 0 \\
\hline$"$ & $4-9 \mathrm{~B}$ & $5: 35: 37.5$ & $-6: 02: 31$ & 0.260 & -0.101 & 0.28 & 0.06 & 12.8 & -111.2 & 0 \\
\hline SMZ 41 & $4-10 \mathrm{~A}$ & 5:35:09.9 & $-6: 03: 53$ & -0.161 & -0.293 & 0.33 & 0.07 & 15.1 & -208.8 & 0 \\
\hline " & $4-10 B$ & $5: 35: 10.7$ & $-6: 03: 43$ & 0.990 & 0.678 & 1.20 & 0.24 & 54.8 & -55.6 & 2 \\
\hline " & $4-10 C$ & $5: 35: 11.1$ & $-6: 03: 34$ & -1.382 & -1.105 & 1.77 & 0.35 & 80.8 & -231.4 & 0 \\
\hline SMZ 49* & $5-23 G$ & $5: 36: 25.7$ & $-6: 35: 30$ & -0.180 & -1.595 & 1.61 & 0.32 & 77.1 & -186.4 & 0 \\
\hline$"$ & 5-23G1 & $5: 36: 25.6$ & $-6: 35: 46$ & -0.280 & -1.863 & 1.88 & 0.38 & 90.1 & -188.5 & 0 \\
\hline " & $5-23 G 2$ & $5: 36: 25.7$ & $-6: 35: 30$ & 0.328 & -1.047 & 1.10 & 0.22 & 52.7 & -162.6 & 2 \\
\hline " & $5-23 \mathrm{G} 3$ & $5: 36: 26.5$ & $-6: 35: 15$ & 0.282 & -1.507 & 1.53 & 0.31 & 73.3 & -169.4 & 0 \\
\hline " & $5-23 \mathrm{G} 4$ & $5: 36: 25.5$ & $-6: 35: 03$ & -0.676 & -1.540 & 1.68 & 0.34 & 80.5 & -203.7 & 0 \\
\hline " & 5-23G5 & $5: 36: 25.6$ & $-6: 34: 55$ & -0.168 & -1.007 & 1.02 & 0.20 & 48.9 & -189.5 & 0 \\
\hline SMZ 49* & $6-4 \mathrm{~A}$ & $5: 36: 32.2$ & $-6: 42: 30$ & -0.998 & -1.479 & 1.78 & 0.36 & 96.5 & -214.0 & 0 \\
\hline " & $6-4 \mathrm{~A} 1$ & $5: 36: 32.6$ & $-6: 42: 11$ & -0.318 & -1.588 & 1.62 & 0.32 & 87.8 & -191.3 & 0 \\
\hline " & $6-4 \mathrm{~A} 2$ & $5: 36: 32.2$ & $-6: 42: 29$ & -0.994 & -1.471 & 1.78 & 0.36 & 96.5 & -214.0 & 0 \\
\hline$"$ & $6-4 \mathrm{~A} 3$ & $5: 36: 32.7$ & $-6: 42: 41$ & -0.514 & -1.740 & 1.81 & 0.36 & 98.1 & -196.5 & 0 \\
\hline " & $6-4 \mathrm{~A} 4$ & $5: 36: 32.3$ & $-6: 42: 50$ & -0.254 & -1.350 & 1.37 & 0.27 & 74.3 & -190.7 & 0 \\
\hline " & $6-4 \mathrm{~A} 5$ & $5: 36: 32.0$ & $-6: 42: 58$ & -0.156 & -2.386 & 2.39 & 0.48 & 129.5 & -183.7 & 0 \\
\hline " & $6-4 \mathrm{~A} 6$ & $5: 36: 33.1$ & $-6: 42: 34$ & -2.380 & 0.907 & 2.55 & 0.51 & 138.2 & 69.1 & 0 \\
\hline " & $6-4 \mathrm{~A} 7$ & $5: 36: 31.7$ & $-6: 42: 44$ & 0.511 & -2.413 & 2.47 & 0.49 & 133.9 & -168.0 & 0 \\
\hline$"$ & $6-4 B$ & $5: 36: 33.6$ & $-6: 44: 14$ & -0.985 & -1.265 & 1.60 & 0.32 & 86.7 & -217.9 & 0 \\
\hline " & $6-4 \mathrm{~B} 1$ & $5: 36: 33.7$ & $-6: 44: 14$ & -0.081 & -1.203 & 1.21 & 0.24 & 65.6 & -183.9 & 0 \\
\hline " & $6-4 \mathrm{~B} 2$ & $5: 36: 34.8$ & $-6: 44: 16$ & -1.784 & -1.035 & 2.06 & 0.41 & 111.7 & -239.9 & 0 \\
\hline " & $6-4 \mathrm{~B} 3$ & $5: 36: 35.7$ & $-6: 44: 26$ & -0.924 & -1.041 & 1.39 & 0.28 & 75.3 & -221.6 & 0 \\
\hline " & $6-4 B 4$ & $5: 36: 34.8$ & $-6: 44: 32$ & 0.132 & -1.588 & 1.59 & 0.32 & 86.2 & -175.2 & 2 \\
\hline$"$ & $6-4 \mathrm{~B} 5$ & $5: 36: 32.9$ & $-6: 43: 55$ & -0.118 & 1.037 & 1.04 & 0.21 & 56.4 & 6.5 & 2 \\
\hline " & $6-4 B 6$ & $5: 36: 33.4$ & $-6: 43: 37$ & 1.572 & -1.667 & 2.29 & 0.46 & 124.1 & -136.7 & 0 \\
\hline " & $6-4 \mathrm{~B} 7$ & $5: 36: 34.8$ & $-6: 43: 38$ & 0.007 & -0.588 & 0.59 & 0.12 & 32.0 & -179.3 & 2 \\
\hline SMZ 74 & $9-1 \mathrm{~A}$ & $5: 40: 23.7$ & $-7: 20: 33$ & 0.208 & 0.376 & 0.43 & 0.09 & 23.3 & -29.0 & 2 \\
\hline " & $9-1 \mathrm{~B}$ & $5: 40: 24.1$ & $-7: 20: 45$ & 0.208 & 0.126 & 0.24 & 0.05 & 13.0 & -58.8 & 2 \\
\hline " & $9-1 \mathrm{C}$ & $5: 40: 26.4$ & $-7: 20: 13$ & -2.110 & 0.583 & 2.19 & 0.44 & 118.7 & 74.6 & 0 \\
\hline " & $9-2 \mathrm{~A}$ & $5: 40: 10.7$ & $-7: 21: 47$ & 0.235 & -2.772 & 2.78 & 0.56 & 150.7 & -175.2 & 0 \\
\hline " & $9-2 B$ & $5: 40: 11.0$ & $-7: 21: 46$ & -1.436 & -0.752 & 1.62 & 0.32 & 87.8 & -242.4 & 0 \\
\hline " & $9-2 \mathrm{C}$ & $5: 40: 10.7$ & $-7: 21: 42$ & 1.331 & 0.434 & 1.40 & 0.28 & 75.9 & -71.9 & 0 \\
\hline SMZ 75* & $9-3 \mathrm{~A}$ & $5: 40: 25.9$ & $-7: 22: 14$ & -0.710 & 0.981 & 1.21 & 0.24 & 65.6 & 35.9 & 0 \\
\hline " & $9-3 \mathrm{~A} 1$ & $5: 40: 26.5$ & $-7: 22: 08$ & -0.872 & 0.377 & 0.95 & 0.19 & 51.5 & 66.6 & 2 \\
\hline " & $9-3 \mathrm{~A} 2$ & $5: 40: 24.9$ & $-7: 22: 22$ & -1.441 & 0.232 & 1.46 & 0.29 & 79.1 & 80.9 & 0 \\
\hline$"$ & $9-3 \mathrm{~A} 3$ & $5: 40: 25.2$ & $-7: 22: 35$ & -0.207 & 1.371 & 1.39 & 0.28 & 75.3 & 8.6 & 0 \\
\hline " & $9-3 \mathrm{~B}$ & $5: 40: 23.5$ & $-7: 22: 47$ & -0.784 & 1.146 & 1.39 & 0.28 & 75.3 & 34.4 & 0 \\
\hline " & $9-3 \mathrm{~B} 1$ & $5: 40: 23.4$ & $-7: 22: 55$ & -1.023 & 1.258 & 1.62 & 0.32 & 87.8 & 39.1 & 0 \\
\hline " & $9-3 \mathrm{~B} 2$ & $5: 40: 23.0$ & $-7: 22: 47$ & -0.747 & 0.627 & 0.98 & 0.20 & 53.1 & 50.0 & 2 \\
\hline SMZ 76 & $9-4 \mathrm{~A} 1$ & $5: 40: 43.8$ & $-7: 23: 30$ & -1.155 & -0.868 & 1.44 & 0.29 & 78.1 & -233.1 & 2 \\
\hline " & $9-4 \mathrm{~A} 2$ & $5: 40: 43.4$ & $-7: 23: 29$ & -1.703 & -0.444 & 1.76 & 0.35 & 95.4 & -255.4 & 0 \\
\hline " & $9-4 \mathrm{~A} 3$ & $5: 40: 43.1$ & $-7: 23: 28$ & 0.983 & 0.522 & 1.11 & 0.22 & 60.2 & -62.0 & 0 \\
\hline " & $9-4 \mathrm{~A} 4$ & $5: 40: 42.3$ & $-7: 23: 28$ & 0.250 & -0.126 & 0.28 & 0.06 & 15.2 & -116.7 & 0 \\
\hline " & $9-4 \mathrm{~A} 5$ & $5: 40: 41.8$ & $-7: 23: 29$ & -0.655 & 0.007 & 0.65 & 0.13 & 35.2 & 89.4 & 2 \\
\hline " & $9-4 \mathrm{~A} 6$ & $5: 40: 40.4$ & $-7: 23: 31$ & -0.497 & 0.716 & 0.87 & 0.17 & 47.2 & 34.8 & 0 \\
\hline " & $9-4 \mathrm{~B} 1$ & $5: 40: 42.5$ & $-7: 23: 45$ & -0.641 & 0.169 & 0.66 & 0.13 & 35.8 & 75.2 & 0 \\
\hline " & $9-4 \mathrm{~B} 2$ & 5:40:41.6 & $-7: 23: 41$ & -0.270 & -0.940 & 0.98 & 0.20 & 53.1 & -196.0 & 0 \\
\hline " & $9-4 \mathrm{~B} 3$ & $5: 40: 40.5$ & $-7: 23: 41$ & -0.108 & 0.313 & 0.33 & 0.07 & 17.9 & 19.0 & 0 \\
\hline " & $9-4 \mathrm{C} 1$ & $5: 40: 39.3$ & $-7: 23: 54$ & -0.060 & 0.283 & 0.29 & 0.06 & 15.7 & 12.0 & 0 \\
\hline " & 9-4D1 & $5: 40: 44.5$ & $-7: 23: 55$ & -1.127 & 1.251 & 1.68 & 0.34 & 91.1 & 42.0 & 0 \\
\hline I & $9-4 \mathrm{D} 2$ & $5: 40: 43.7$ & $-7: 23: 52$ & 1.161 & 0.474 & 1.25 & 0.25 & 67.8 & -67.8 & 0 \\
\hline
\end{tabular}


Table B.1. continued.

\begin{tabular}{|c|c|c|c|c|c|c|c|c|c|c|}
\hline Flow & Knot & $\begin{array}{l}\text { RA } \\
(2000)\end{array}$ & $\begin{array}{l}\text { Dec } \\
(2000)\end{array}$ & $\begin{array}{r}\text { shift- } x \\
\text { (pix) }\end{array}$ & $\begin{array}{r}\text { shift-y } \\
\text { (pix) }\end{array}$ & $\begin{array}{l}\text { shift } \\
\text { (pix) }\end{array}$ & $\begin{array}{r}\text { shift } \\
(\operatorname{arcsec})\end{array}$ & $\begin{array}{r}\mathrm{Vel}^{a} \\
\left(\mathrm{~km} \mathrm{~s}^{-1}\right)\end{array}$ & $\begin{array}{r}\mathrm{PA}^{b} \\
(\mathrm{deg})\end{array}$ & $F^{c}$ \\
\hline SMZ 76* & $9-6 \mathrm{~A}$ & $5: 40: 15.6$ & $-7: 24: 29$ & -1.614 & 0.891 & 1.84 & 0.37 & 99.7 & 61.1 & $\overline{0}$ \\
\hline 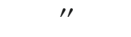 & $9-6 \mathrm{~A} 1$ & $5: 40: 15.4$ & $-7: 24: 24$ & -0.966 & -0.029 & 0.97 & 0.19 & 52.6 & -268.3 & 2 \\
\hline " & $9-6 \mathrm{~A} 2$ & $5: 40: 16.8$ & $-7: 24: 32$ & -0.906 & 0.822 & 1.22 & 0.24 & 66.1 & 47.8 & 0 \\
\hline " & $9-6 A 3$ & $5: 40: 15.3$ & $-7: 24: 31$ & -2.058 & -1.715 & 2.68 & 0.54 & 145.3 & -230.2 & 0 \\
\hline " & $9-6 \mathrm{~B} 1$ & $5: 40: 18.0$ & $-7: 24: 25$ & -1.591 & -0.904 & 1.83 & 0.37 & 99.2 & -240.4 & 2 \\
\hline " & $9-6 \mathrm{~B} 2$ & $5: 40: 17.7$ & $-7: 24: 21$ & 0.553 & 1.283 & 1.40 & 0.28 & 75.9 & -23.3 & 0 \\
\hline$"$ & $9-6 \mathrm{C}$ & $5: 40: 18.9$ & $-7: 25: 06$ & -0.491 & 0.630 & 0.80 & 0.16 & 43.4 & 37.9 & $\mathrm{O}$ \\
\hline " & $9-6 \mathrm{D} 1$ & $5: 40: 17.7$ & $-7: 24: 37$ & -1.504 & -0.506 & 1.59 & 0.32 & 86.2 & -251.4 & 0 \\
\hline " & $9-6 \mathrm{D} 2$ & $5: 40: 17.3$ & $-7: 24: 38$ & -0.997 & 0.588 & 1.16 & 0.23 & 62.9 & 59.5 & 0 \\
\hline " & $9-6 E$ & $5: 40: 17.8$ & $-7: 24: 58$ & -0.198 & 0.716 & 0.74 & 0.15 & 40.1 & 15.5 & 0 \\
\hline " & $9-6 \mathrm{~F}$ & $5: 40: 16.5$ & $-7: 24: 40$ & -1.307 & 0.654 & 1.46 & 0.29 & 79.1 & 63.4 & 0 \\
\hline DFS 107 & $3-7 A$ & $5: 34: 32.0$ & $-5: 37: 51$ & 0.864 & 1.225 & 1.50 & 0.30 & 81.3 & -35.2 & 2 \\
\hline " & $3-7 \mathrm{~B}$ & $5: 34: 31.3$ & $-5: 37: 49$ & 1.687 & 0.814 & 1.87 & 0.37 & 101.4 & -64.2 & 0 \\
\hline DFS 108 & $3-1 \mathrm{~A}$ & $5: 35: 04.3$ & $-5: 33: 30$ & -0.917 & -0.108 & 0.92 & 0.18 & 49.9 & -263.3 & 0 \\
\hline DFS 109 & $3-5$ & $5: 35: 23.6$ & $-5: 36: 09$ & -1.339 & 0.230 & 1.36 & 0.27 & 73.7 & 80.3 & 0 \\
\hline DFS 111 & $3-11$ & $5: 34: 46.5$ & $-5: 40: 14$ & 0.399 & -1.531 & 1.58 & 0.32 & 85.6 & -165.4 & 0 \\
\hline DFS $124^{*}$ & $6-16 A$ & $5: 36: 33.1$ & $-6: 53: 25$ & -0.443 & -0.845 & 0.95 & 0.19 & 51.5 & -207.7 & 0 \\
\hline " & 6-16Ae & $5: 36: 33.3$ & $-6: 53: 25$ & -1.245 & -0.798 & 1.48 & 0.30 & 80.2 & -237.3 & 0 \\
\hline " & 6-16Aw & $5: 36: 33.0$ & $-6: 53: 25$ & 0.026 & -1.233 & 1.23 & 0.25 & 66.7 & -178.8 & 0 \\
\hline DFS 131 & $9-7 \mathrm{~A}$ & $5: 40: 21.4$ & $-7: 27: 17$ & -1.415 & 0.998 & 1.73 & 0.35 & 93.8 & 54.8 & 0 \\
\hline " & $9-7 \mathrm{~B}$ & $5: 40: 20.6$ & $-7: 27: 21$ & -1.036 & -0.235 & 1.06 & 0.21 & 57.5 & -257.2 & 0 \\
\hline " & $9-7 \mathrm{C}$ & $5: 40: 21.7$ & $-7: 27: 10$ & -1.000 & 0.147 & 1.01 & 0.20 & 54.7 & 81.6 & 2 \\
\hline
\end{tabular}

${ }^{a}$ Tangential velocity. A nominal error of $21 \mathrm{~km} \mathrm{~s}^{-1}$ has been adopted (see text for details).

${ }^{b}$ Position Angle of the PM vector, measured E of N.

${ }^{c}$ Status Flag. Those with value 2 are uncertain.

${ }^{*}$ In these objects clusters of knots are labelled A, B, etc., with the individual knots themselves labelled A1, A2, etc. and B1, B2, etc. The PM of 5-23 G, for example, is for all knots G1-G5 measured together.

uncertain. 1-19A appears to be moving northward away from IRS 11, so could certainly be part of the SMZ 11 flow.

\section{B.1.5. SMZ 14}

Knots $1-20 \mathrm{~A}$ and $1-20 \mathrm{~B}$ are possibly outer bows in a flow driven by IRS 14 (with SMZ 16 as the counter-lobe). Fainter features (1-25, 1-28 and 1-29) exist closer to the source, though we have no PM measurements for these objects. The PMs for 1-20 are roughly northward, as expected, though they are relatively uncertain.

\section{B.1.6. SMZ 17/18}

This spectacular flow comprises a bright outer bow 1-27A and a curving filament $1-27 \mathrm{C}+\mathrm{D}$. These delineate the northern and western edges of a cavity, respectively. 1-27B is a bright knot on the eastern side of the cavity that may be associated with a second flow (SMZ 18). The PMs of these features support excitation by one of the protostars to the south; we identify IRS 17 as the best candidate (see Fig. 2 and main text).

\section{B.2. $O M C 2$ and $O M C 4$}

The PMs of $\mathrm{H}_{2}$ features in OMC 2 (SMZ 21 to SMZ 25) are displayed in Fig. B.2; values for the remaining $\mathrm{H}_{2}$ flows in this section, SMZ 31-SMZ 36 and DFS 107, 108, 109 and 111, all of which are located in the OMC 4 region (south of OMC 1), are given in Table B.1.

\section{B.2.1. SMZ 21/22}

The knots 1-37A, B and C, previously identified with two separate $\mathrm{H}_{2}$ flows SMZ 21 and SMZ 22, probably form part of a single outflow. All three features have well-defined PMs to the south, away from IRS 21.

\section{B.2.2. SMZ 23}

This $\mathrm{H}_{2}$ flow comprises a pointed, knotty bow (1-38A) and a collimated chain of much fainter knots (1-38B to E). Motion to the south is certainly implied by the flow morphology. However, the measured PMs are relatively uncertain, being heavily influenced by gradients in the background nebulosity. Even so, the source could still be near IRS 23, which is very well aligned with the flow axis.

\section{B.2.3. $S M Z 24$}

The PMs of the $\mathrm{H}_{2}$ knots along this collimated flow (1-40 A,B,C) clearly point to IRS 24 being the driving source; all features are essentially moving towards the southwest. 1-40A is the brightest and most compact feature; it is more-or-less coincident with the IRS source. $1-40 \mathrm{~A} 1$ is the cap of a bow situated $\sim 20^{\prime \prime}$ south-west of the source, respectively. $1-40 \mathrm{~B}$ and $1-40 \mathrm{C}$ comprise a knotty bow a further arcminute downwind.

\section{B.2.4. SMZ 25}

This flow comprises an outer bow shock (1-32A) and an $\mathrm{H}_{2}$ arc (1-39A) that presumably traces the eastern edge of a second bow located closer to the source. Knots B and C in each object are fainter sub-components. 1-39A has a PM consistent with motion 


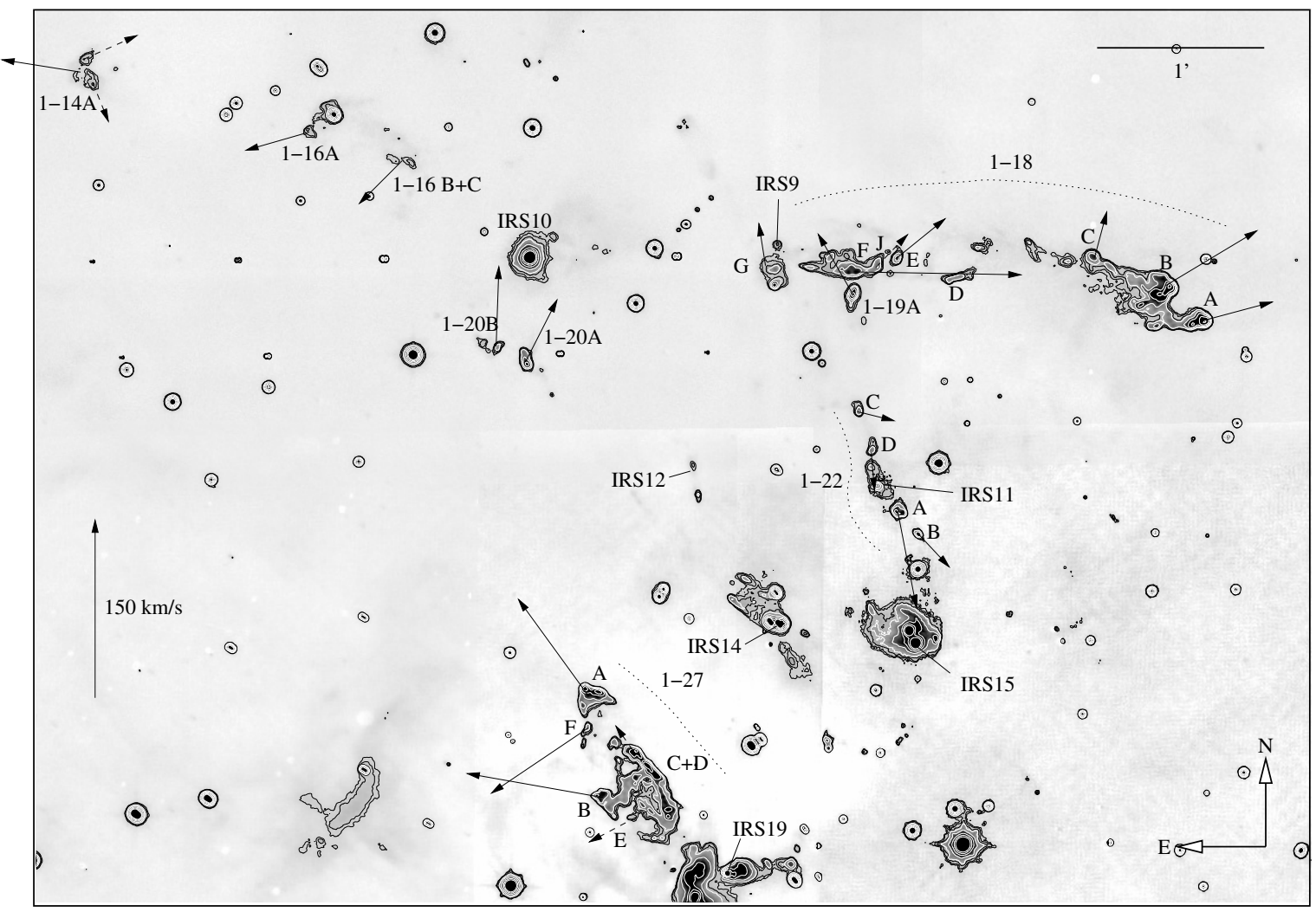

Fig. B.1. $\mathrm{H}_{2}$ image of OMC 3 with proper motion vectors marked for SMZ 8 (1-14), SMZ 9 (1-18), SMZ 10 (1-16), SMZ 11/13 (1-22), SMZ 14 (1-20) and SMZ 17/18 (1-27). The knot nomenclature follows Sta02 (see also Table B.1). Candidate outflow sources are labelled.

towards 1-32A, so both are almost certainly part of the same outflow, with the source situated to the south-southeast. The remaining features in 1-32 and 1-39 exhibit low and/or random motions, so overall the results for this flow are inconclusive.

\section{B.2.5. SMZ 31}

3-9A and 3-9B are $\mathrm{H}_{2}$ knots in the northern lobe of a compact, bipolar flow centred on IRS 31. Both features seem to be moving toward the north-northwest, consistent with this association.

\section{B.2.6. SMZ 32}

The PMs of the faint $\mathrm{H}_{2}$ knots in this flow (3-8 north and south, and 3-10) are rather uncertain, although they do suggest that these features are part of the same north-south flow. The source location is uncertain.

\section{B.2.7. SMZ 33}

Knots 3-12A and 3-12C are compact $\mathrm{H}_{2}$ features with PMs roughly to the north/northwest. The association with IRS 33 is tentative.

\section{B.2.8. SMZ 34}

3-13A has a PM directed roughly northward, consistent with its association with the much fainter knot 3-13B.

\section{B.2.9. SMZ 35}

Knot $3-14$ is a very compact feature $\sim 1^{\prime}$ east-northeast of IRS 35. Its modest eastward PM is not inconsistent with this Spitzer protostar being the exciting source.

\section{B.2.10. SMZ 36}

This $\mathrm{H}_{2}$ flow comprises three very faint knots in a compact group, 3-15A, 3-15B and 3-15C; $\mathrm{A}$ is the brightest. They are aligned along a roughly northeast-southwest axis, although their PMs are inconclusive and Spitzer revealed no obvious candidate driving source.

\section{B.2.11. DFS 107}

This flow consists of a very faint though well-collimated $\mathrm{H}_{2}$ jet (3-7A) that points to a bright knot (3-7B). The protostar IRS 107 lies on the same axis, although the PM of 3-7B is toward this source (Table B.1), rather than away from it.

\section{B.2.12. DFS 108}

3-1 A is a bright $\mathrm{H}_{2}$ knot in a region of diffuse nebulosity which may well influence the PM measurement. This probably explains why its PM appears to be to the east, "toward" the nearest Spitzer protostar (IRS 108).

\section{B.2.13. DFS 109}

The brightest $\mathrm{H}_{2}$ feature in this faint, collimated flow is knot 3-5. This bow-shaped knot has a well-defined PM to the east, consist 
C. J. Davis et al.: A census of molecular hydrogen outflows and their sources along the Orion A molecular ridge, Online Material $p$ 18

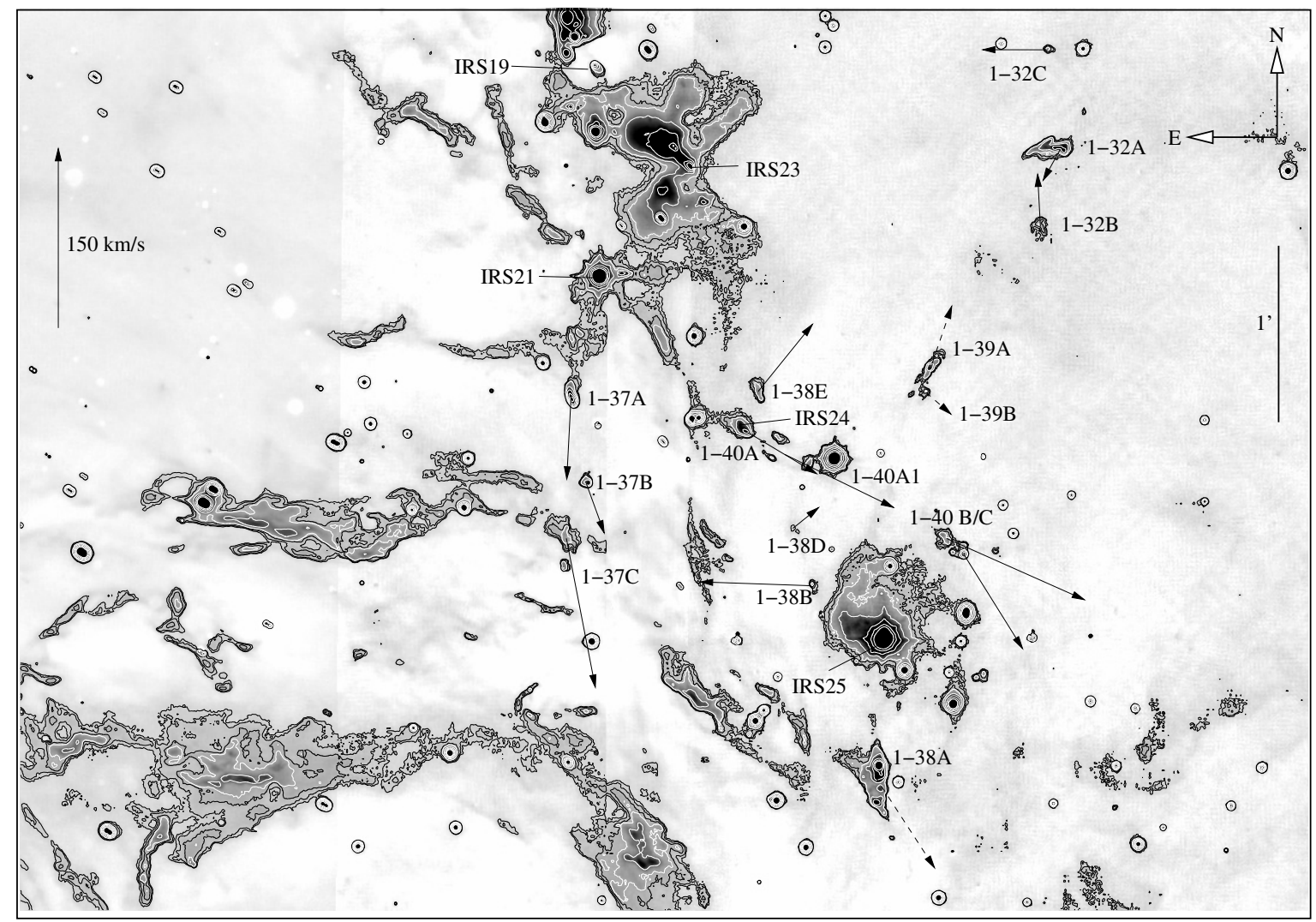

Fig. B.2. $\mathrm{H}_{2}$ image of OMC 2 with proper motion vectors marked for SMZ 21/22 (1-37), SMZ 23 (1-38), SMZ 24 (1-40) and SMZ 25 (1-32, 1-39).

with the Spitzer source/MAMBO core IRS 109 being the driving source.

\section{B.2.14. DFS 111}

Knot 3-11 appears to be a small $\mathrm{H}_{2}$ bow situated $10^{\prime \prime}-20^{\prime \prime}$ northeast of a Spitzer disc source and a protostar. However, its relatively well-defined PM is towards both sources, so the association with either is questionable.

\section{B.3. OMC 5 (the NGC 1980 region)}

The PMs of the $\mathrm{H}_{2}$ features that comprise SMZ 37, SMZ 38 and SMZ 39 are displayed in Fig. B.3.

\section{B.3.1. SMZ 37}

3-16 (A,B) and 3-17 (A,B,C) are located near a pair of dust cores and two Spitzer sources, although they do not appear to be driven by either source. Sta02 associate these two groups of knots with flow SMZ 37. However, the features are not well aligned, and the northward PMs of 3-17A, B and C suggest an association with SMZ 38.

\section{B.3.2. SMZ 38}

This is a long, curving flow comprising $\mathrm{H}_{2}$ objects 4-1, 4-2 (A,B), 4-3 and possibly parts of 4-5 and (to the north) $3-17$. The flow is associated with a cold dust core and the Spitzer protostar IRS 38. The PMs of the many $\mathrm{H}_{2}$ knots along this flow are consistent with this interpretation; the bright knots in the northern lobe in particular (4-1, 4-2 and possibly also 3-17) are clearly moving northward. The situation in the southern counter-lobe, where SMZ 38 crosses the orthogonal flow SMZ 39 near features 4-5, 4-6 and 4-7, is less clear.

\section{B.3.3. SMZ 39}

This flow crosses SMZ 38 in the vicinity of knots 4-5, 4-6 and 4-7. The flow is driven by IRS 39 (not shown in Fig. B.3) and its associated dust core, and includes knots that comprise 4-4 $3^{\prime}$ to the northeast. The PMs of 4-4 sub-features A, B and D clearly support this interpretation. Object 4-6 is also probably part of this flow, as is $4-5 \mathrm{~A}, 4-5 \mathrm{~B}$ and $4-5 \mathrm{D}$. The arcuate knot $4-5 \mathrm{C}$ is probably part of SMZ 38, described in the previous section. The association of knot 4-7 is unclear.

\section{B.4. L 1641-N}

The PMs of a few unrelated $\mathrm{H}_{2}$ knots, and some of the features in the huge SMZ 49 flow, are displayed in Fig. B.4. These are discussed in this section.

\section{B.4.1. SMZ 40}

The PMs of the components that comprise the bright though compact $\mathrm{H}_{2}$ object 4-9 are unclear, so the association of this object with the nearby Spitzer young star IRS 40 (labelled in Fig. 5) is again tentative. 
C. J. Davis et al.: A census of molecular hydrogen outflows and their sources along the Orion A molecular ridge, Online Material p 19

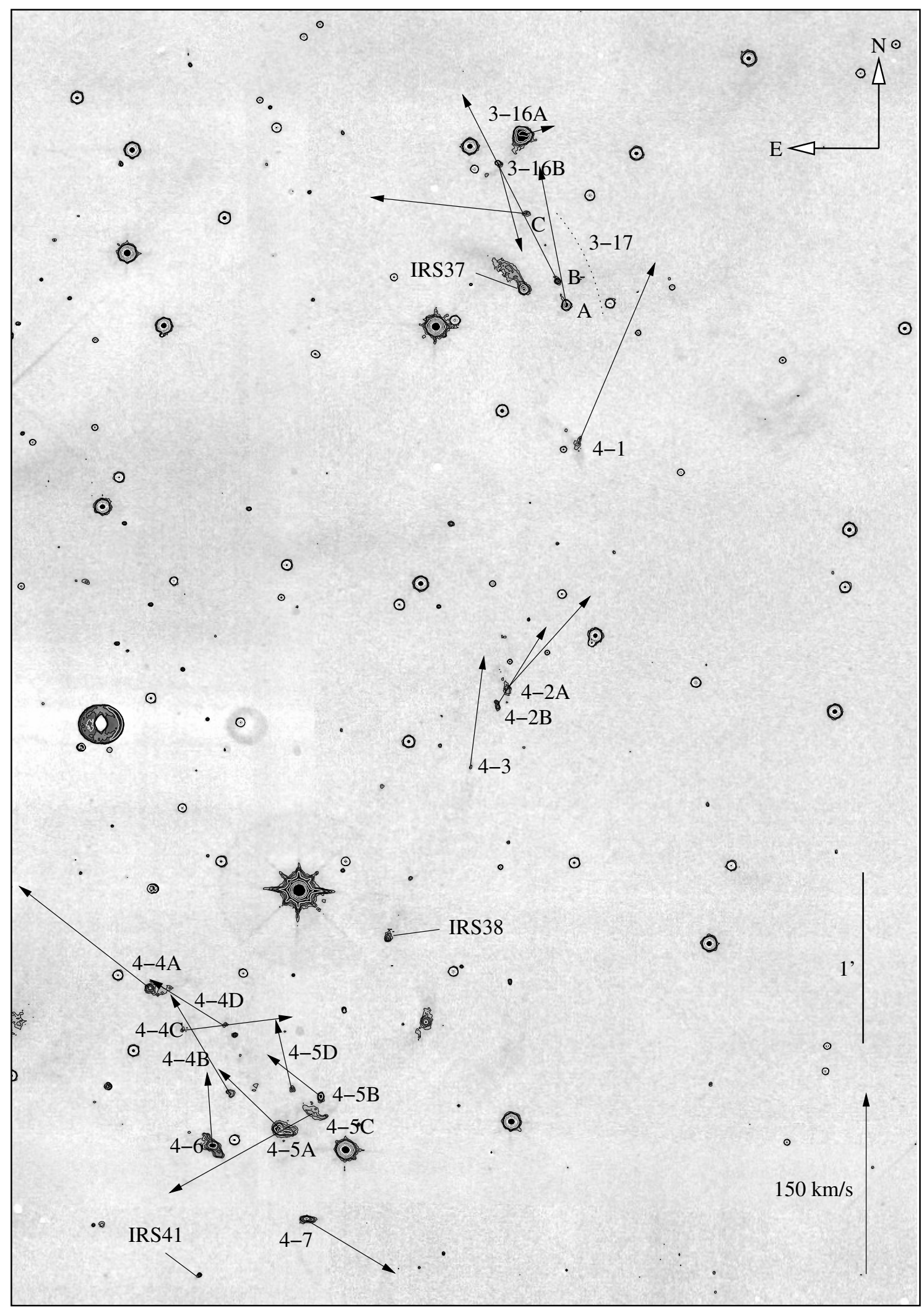

Fig. B.3. $\mathrm{H}_{2}$ image of jets SMZ 37 (3-16, 3-17), SMZ 38 (4-1, 4-2, 4-3) and SMZ 39 (4-4 to 4-7) with proper motion vectors marked.

\section{B.4.2. SMZ 41}

The morphology of 4-10 certainly suggests motion to the south, away from the dusty core and Spitzer protostar IRS 41 (labelled in Fig. 5). However, the PMs of the components that comprise this $\mathrm{H}_{2}$ feature are inconclusive.

\section{B.4.3. SMZ 49}

The southern lobe of the giant SMZ 49 outflow comprises a number of large, sweeping bow shocks. The shapes of these features cast little doubt on their direction of motion away from L 1641-N. However, some of the brightest $\mathrm{H}_{2}$ features in 
C. J. Davis et al.: A census of molecular hydrogen outflows and their sources along the Orion A molecular ridge, Online Material p 20

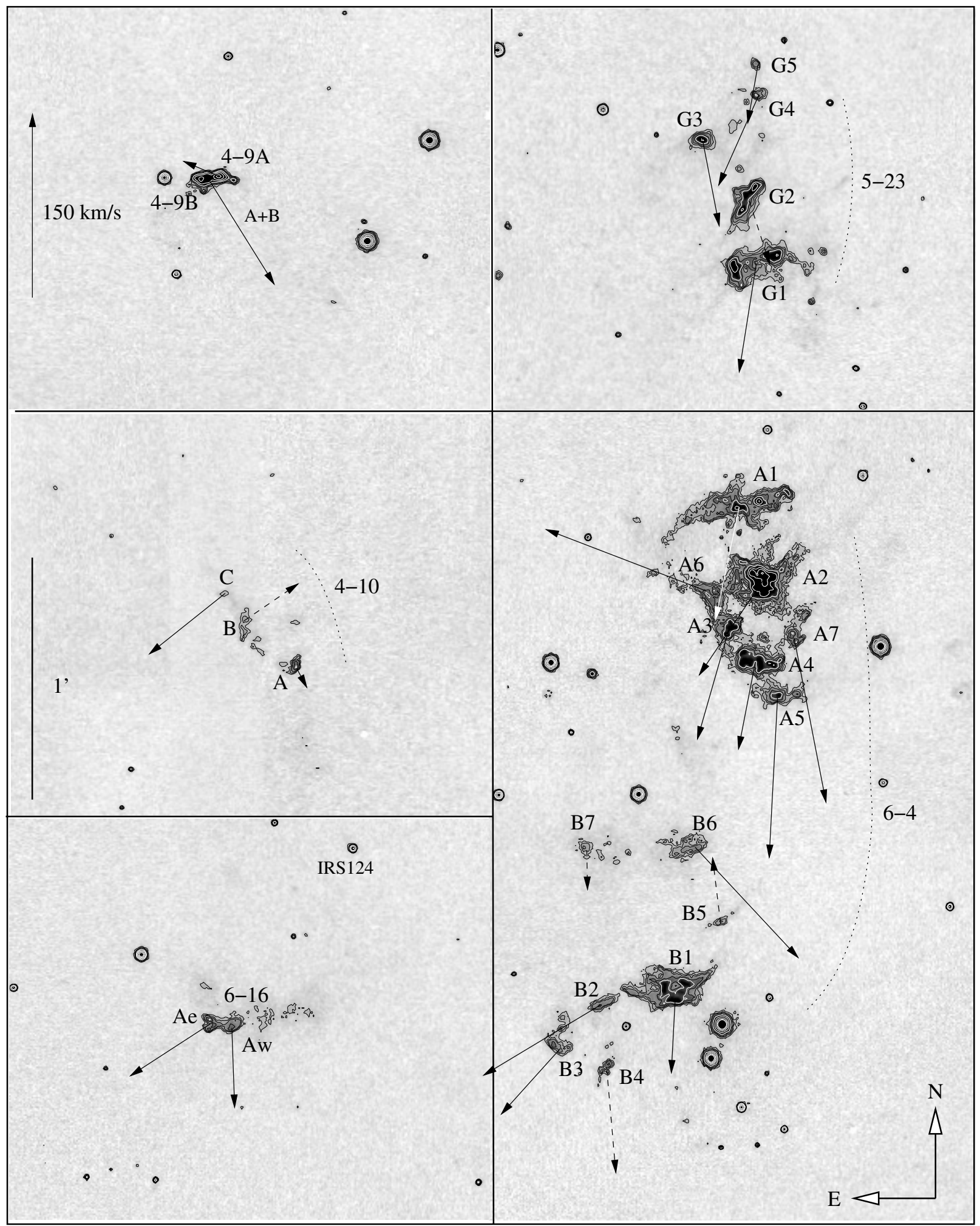

Fig. B.4. Left: $\mathrm{H}_{2}$ images of features SMZ 40 (4-9), SMZ 41 (4-10) and DFS 124 (6-16) with PMs indicated. Right: $\mathrm{H}_{2}$ images of portions of the SMZ 49 outflow south of L 1641-N (5-23 and 6-4) with PM vectors marked.

SMZ 49 have more complex morphologies that suggest a possible association with other outflows. We have therefore measured the PMs of two groups of features, $5-23 \mathrm{G}$ (situated $\sim 14^{\prime}$ south of $\mathrm{L} 1641-\mathrm{N}$ ) and $6-4 \mathrm{~A}$ and $\mathrm{B}$ (found a further $6^{\prime}-8^{\prime}$ downwind/to the south) - see Fig. B.4. Feature 5-23G in particular has a shape that hints at excitation from a source to the southeast (perhaps V380 Ori-NE). Even so, we find that, overall, the $\mathrm{H}_{2}$ knots in both regions are moving southward, and thus confirm the association of 5-23G, 6-4A and 6-4B with the rest of the SMZ 49 outflow. 


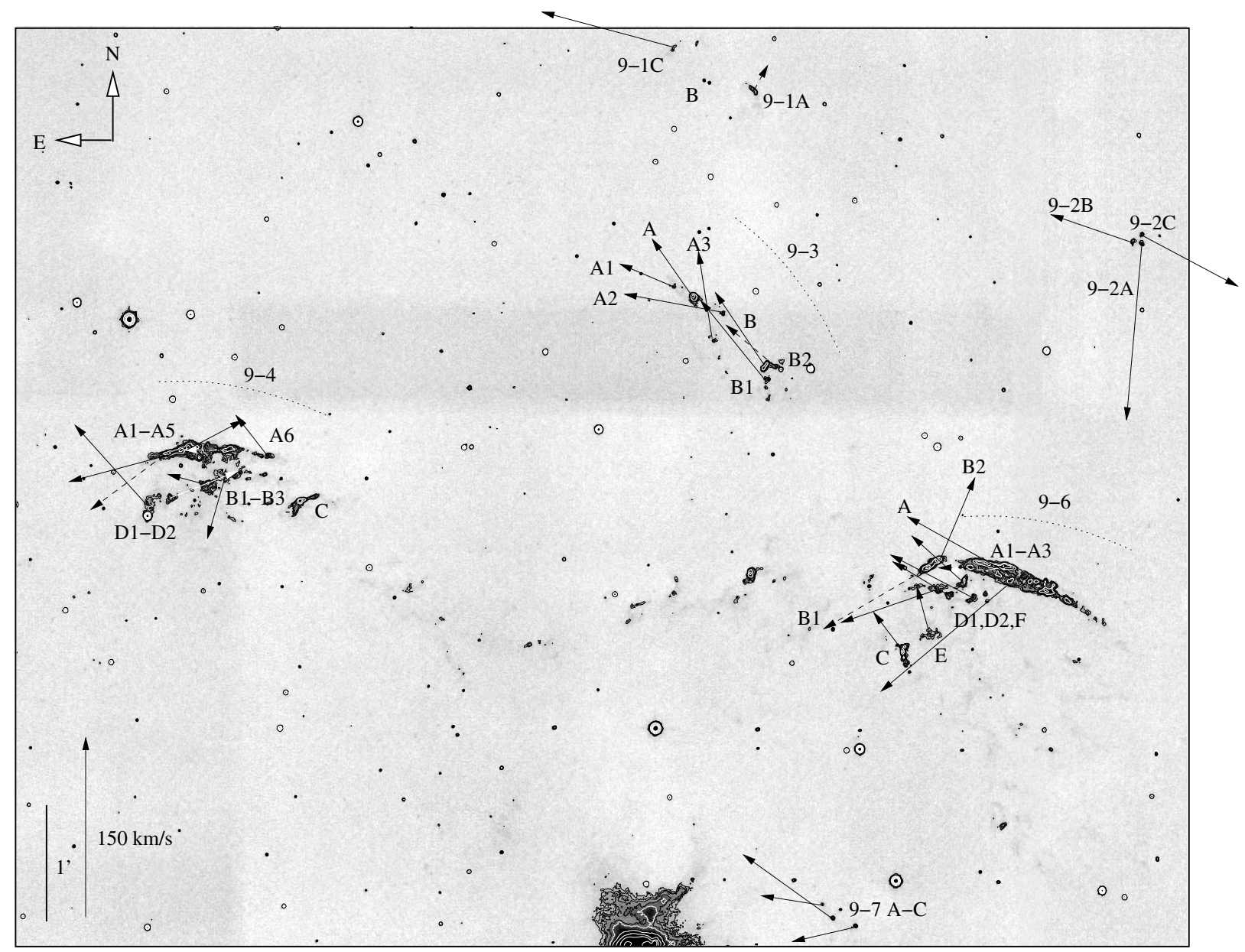

Fig. B.5. $\mathrm{H}_{2}$ images of features SMZ 74 (9-1, 9-2), SMZ 75 (9-3), SMZ 76 (9-4, 9-6) and DFS 131 (9-7) with proper motion vectors marked.

\section{B.4.4. DFS 124}

6-16 is a bright, bow shaped $\mathrm{H}_{2}$ feature $\sim 48^{\prime}$ south of $\mathrm{HH} 1 / 2$. However, there is a Spitzer protostar that is much closer and is arguably the more likely driving source. The PMs of the two main knots in 6-16 support this association (though of course $\mathrm{HH} 1 / 2$ and its source are also to the north): 6-16 is not only moving away from IRS 124, but appears to be spreading outwards.

\section{B.5. L 1641-C/Re 50}

The molecular outflows SMZ 74-SMZ 76 and DFS 131 are displayed in Fig. B.5, with PM vectors marked.

\section{B.5.1. SMZ 74}

Objects 9-1 and 9-2 could form part of one long flow, although these compact features are very faint and their PMs are generally rather uncertain. 9-1C, at the northeastern end of this object, has the best-defined PM: it appears to be moving towards the north-northeast. No source candidate was identified nearby in the Spitzer data.

\section{B.5.2. SMZ 75}

The PMs of the bow-shaped $\mathrm{H}_{2}$ features 9-3A and 9-3B (and fainter sub-components A1, A2, A3 and B1, B2, B3) are all directed toward the northeast, consistent with these two groups of features being part of the same flow, driven by one of the Spitzer protostars found $5^{\prime}-6^{\prime}$ to the southwest.

\section{B.5.3. SMZ 76}

SMZ 76 comprises two large, complex arcs, denoted 9-4 and 9-6 by $\mathrm{Sta} 02$. Although the sub-components exhibit considerable scatter in their PMs, and measurements for individual $\mathrm{H}_{2}$ knots are influenced in some cases by neighbouring features, overall, 9-4 and 9-6 both appear to be moving towards the east or northeast, consistent with them being part of the same flow that is driven by one of the many Spitzer sources to the south-west. IRS 76 and its associated dense core is the best candidate.

\section{B.5.4. DFS 131}

The three compact $\mathrm{H}_{2}$ knots that comprise feature 9-7 are probably not part of the large SMZ 76 outflow. Instead, their welldefined PMs to the east/north-east suggest a driving source to the west: IRS 131, which is also coincident with a dust core, is the best candidate (see Fig. 12). 